\title{
Community of Practice: A Path to Strategic Learning
}

N. M. Carlson

April 2003 
INEEL/EXT-03-01405

\section{Community of Practice: A Path to Strategic Learning}

Nancy M. Carlson

April 2003

Idaho National Engineering and Environmental Laboratory Idaho Falls, Idaho 83415

Prepared for the

U.S. Department of Energy

Under DOE Idaho Operations Office

Contract DE-AC07-99ID13727 


\begin{abstract}
To explore the concept of community of practice, the research initially concentrates on a strategic business process in a research and applied engineering laboratory discovering essential communication tools and processes needed to cultivate a high functioning cross-disciplinary team engaged in proposal preparation. Qualitative research in the human ecology of the proposal process blends topic-oriented ethnography and grounded theory and includes an innovative addition to qualitative interviewing, called meta-inquiry. Meta-inquiry uses an initial interview protocol with a homogeneous pool of informants to enhance the researcher's sensitivity to the unique cultures involved in the proposal process before developing a formal interview protocol.

In this study the preanalysis process uses data from editors, graphic artists, text processors, and production coordinators to assess, modify, enhance, and focus the formal interview protocol with scientists, engineers, and technical managers-the heterogeneous informants. Thus this human ecology-based interview protocol values homogeneous and heterogeneous informant data and acquires data from which concepts, categories, properties, and both substantive and formal theory emerges.

The research discovers the five essential processes of owning, visioning, reviewing, producing, and contributing for strategic learning to occur in a proposal community of practice. The apprenticeship, developmental, and nurturing perspectives of adult learning provide the
\end{abstract}


proposal community of practice with cohesion, interdependence, and caring, while core and boundary practices provide insight into the tacit and explicit dimensions of the proposal process. By making these dimensions explicit, the necessary competencies, absorptive capacity, and capabilities needed for strategic learning are discovered.

Substantive theory emerges and provides insight into the ability of the proposal community of practice to evolve, flourish, and adapt to the strategic advantage of the laboratory. The substantive theory explores the dimensions of owning, visioning, reviewing, producing, and contributing and their interrelationship to community learning dynamics. Through dialogue, creative tension, and imagination, the proposal community of practice focuses on actionable goals linked by proactively participating in practice, creating possibilities, evaluating and enhancing potential, producing a valued product, and confirming strategic value. Lastly, a formal theory emerges linking competency-capacity-capability, cohesion, interdependence, and caring as essential attributes of strategic learning communities. 


\section{FOREWORD}

This document was originally prepared as a dissertation in partial

fulfillment of the requirements for the degree of Doctor of Philosophy with a major in Education from the University of Idaho. 
Community of Practice vi 


\section{ACKNOWLEDGMENTS}

My deepest appreciation to all the members of the virtual proposal service team for making this research possible. Your dedication in crafting persuasive text, graphics, and lay-ups and in assisting the talented scientists and engineers at the laboratory convey their innovative concepts demonstrates the power of a caring community of practice. In addition to the proposal team, I am grateful to all the scientists, engineers, and managers who have interacted with the team over the past three years and wish to express a special thanks to those proposal team members, scientists, engineers, and managers who participated as informants in this research.

To my committee members, my gratitude for your encouragement and guidance throughout the research process. I extend a special thanks to my mentor, Mark McCaslin, who inspired me to explore grounded theory as a research methodology.

I would like to recognize the dedicated assistance of Beth for spending nights and weekends transcribing interview tapes and books; Allen and Chris for stunning graphics; Penny for formatting; and Jade for providing insight and counsel. Without your assistance, this manuscript would have been a far more stressful process. 
Community of Practice viii 


\section{CONTENTS}

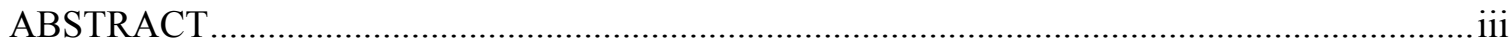

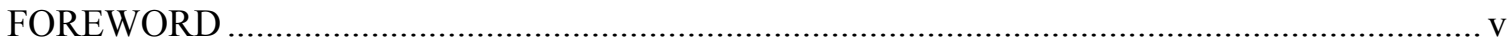

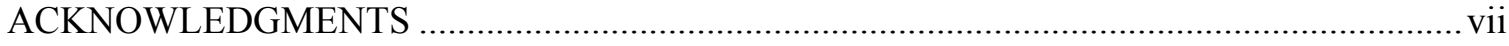

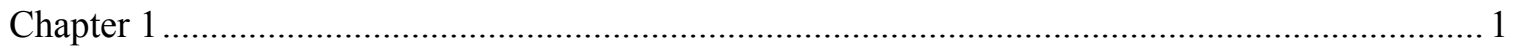

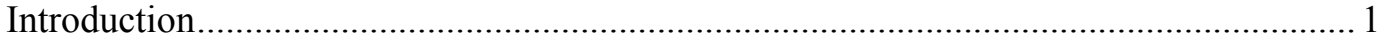

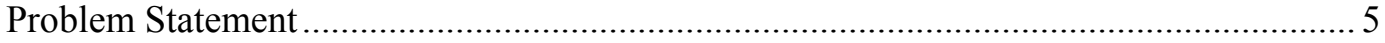

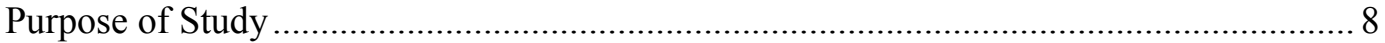

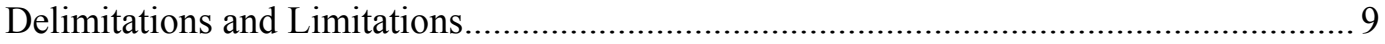

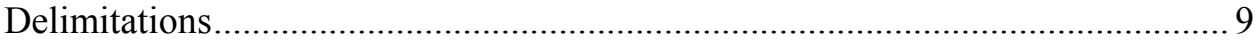

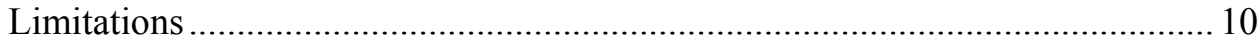

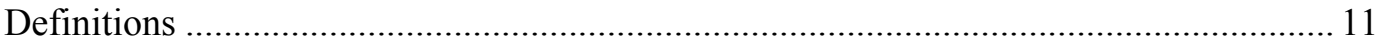

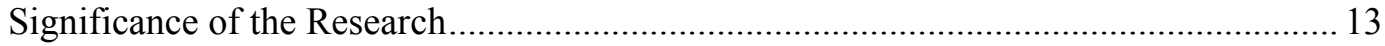

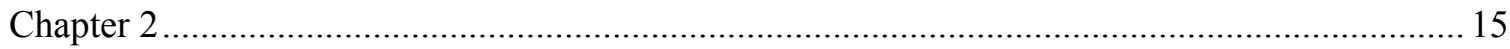

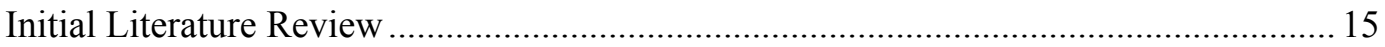

Forming a Strategic Learning Community .................................................................. 17

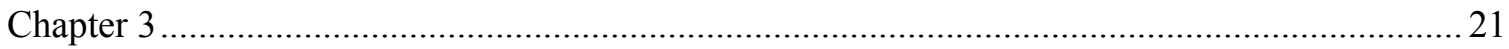

Assumptions and Rationale for the Use of Ethnography and Grounded Theory .............. 21

Homogeneous Informant Interview Process ............................................................. 24

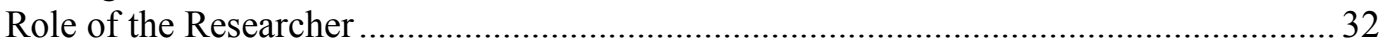

Approach for Developing the Grand Tour Question .................................................. 35

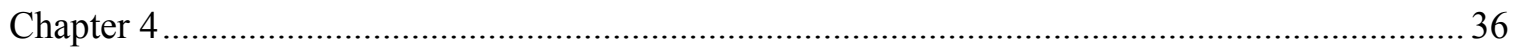

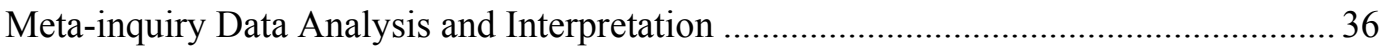

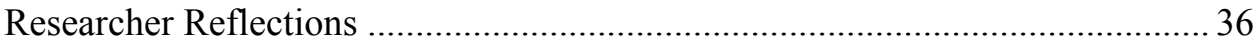

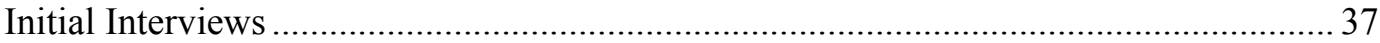

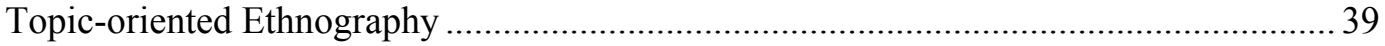

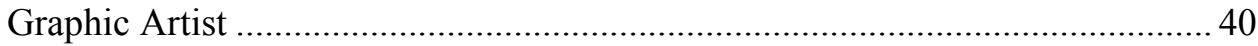

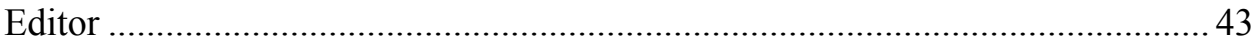

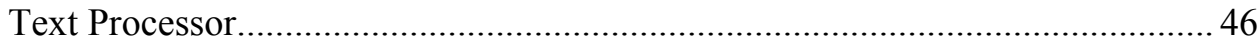

Topic-oriented Ethnography Summary of Homogeneous Informant Data ........................ 48

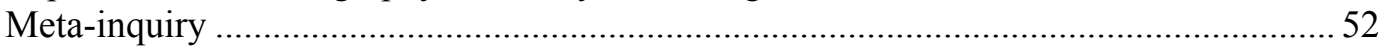

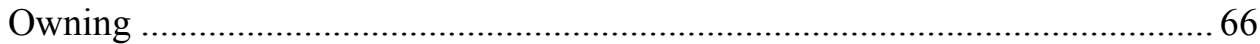

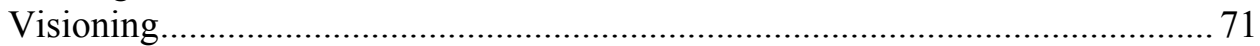

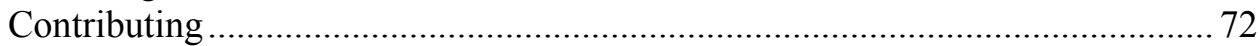

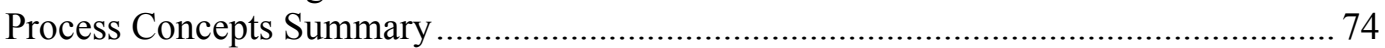

Core Team Attributes of a Community of Practice ........................................................... 76

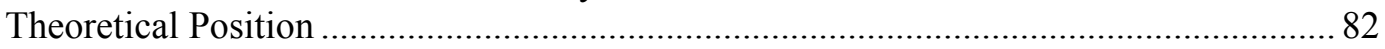

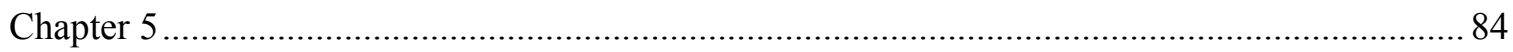

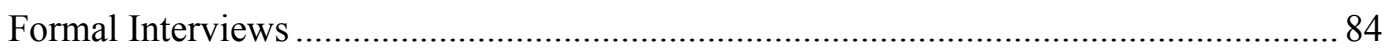

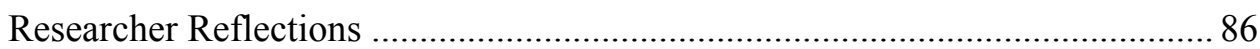

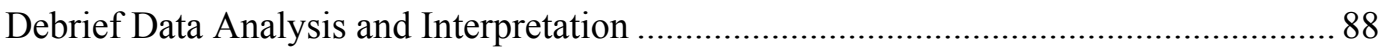

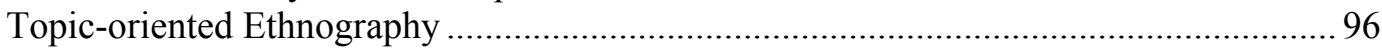




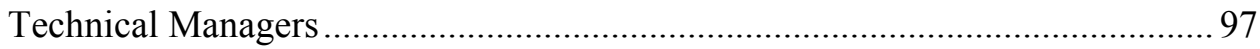

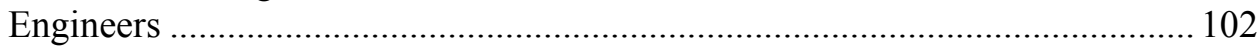

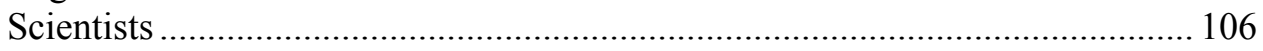

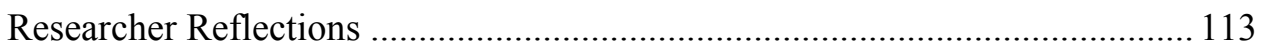

Topic-oriented Ethnography Summary of Heterogeneous Informant Interviews............ 114

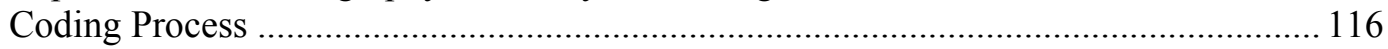

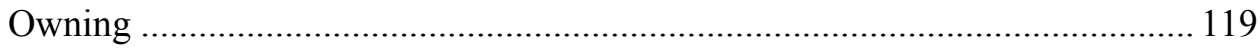

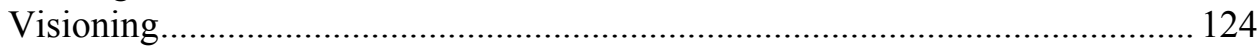

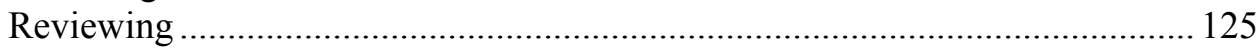

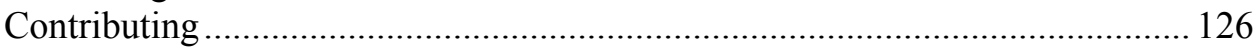

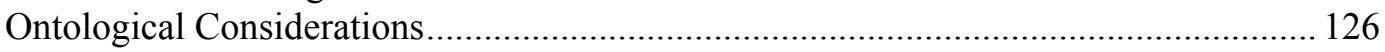

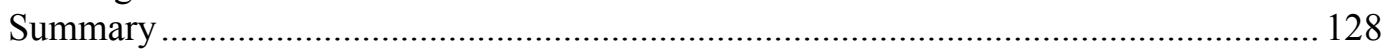

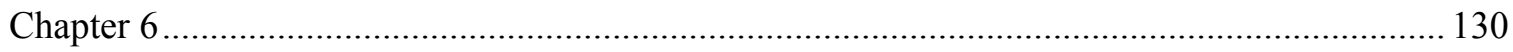

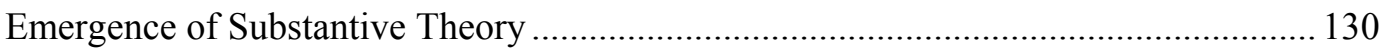

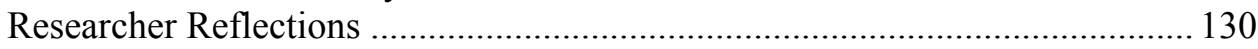

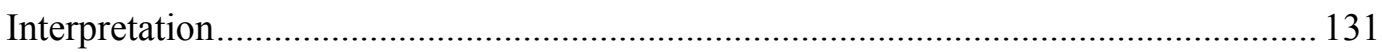

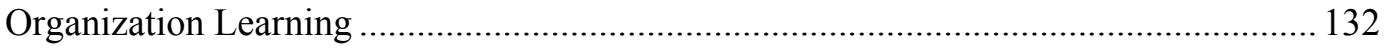

Human Ecosystem-Core and Boundary Practices ..................................................... 138

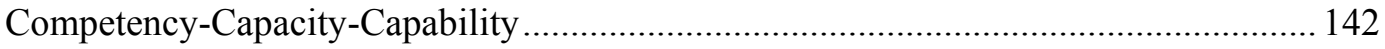

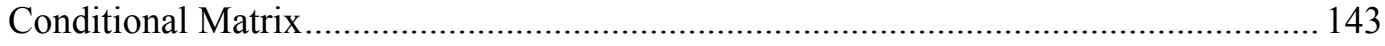

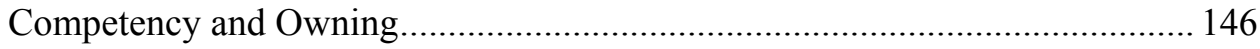

Visioning, Reviewing, and Producing - The Capacity Potentiator ................... 148

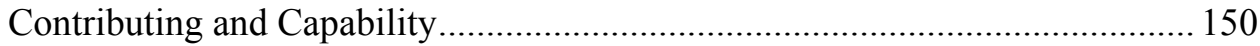

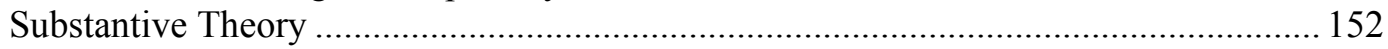

The Proposal Team as a Strategic Community of Practice.............................. 154

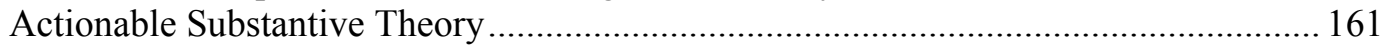

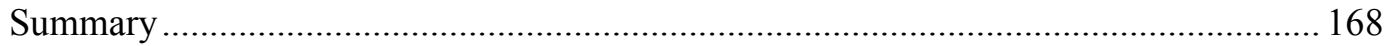

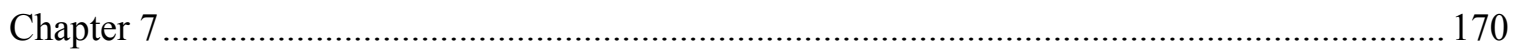

Implications of Meta-inquiry and Community of Practice to Formal Theory ................ 170

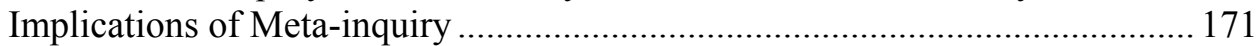

Valuing the Substantive Theory - External Validity ....................................... 173

Community of Practice Adjourning Impacts ................................................. 177

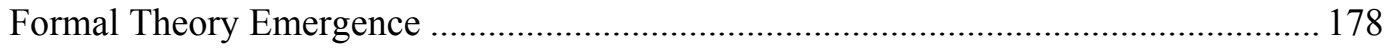

Extant Literature Studies of Practice …........................................................ 179

Implications for Strategic Learning ............................................................... 187

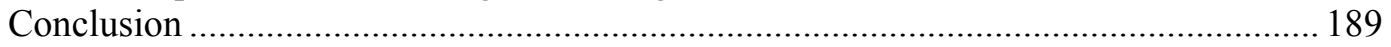

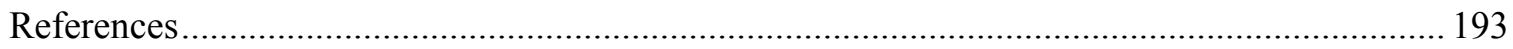

\section{FIGURES}

1. Strategic organization focuses critical processes achieving a competitive advantage ......... 2

2. Work flow of the current proposal process ................................................................... 7

3. Levels of participation in a community of practice (Wenger, 2000a) .............................. 18

4. The position of meta-inquiry in the interview process …............................................... 22 
5. The six stages of grounded theory including meta-inquiry .......................................... 28

6. The author provides a concept; the editor provides structure; the artist provides form;

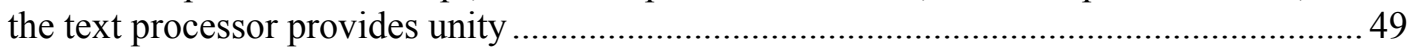

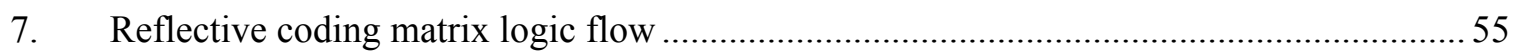

8. Emergence of meta-inquiry process concepts using the conditional relationship and

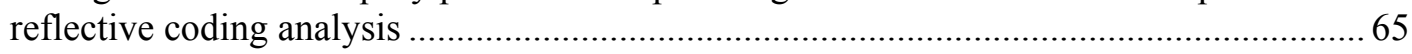

9. Homogeneous informants view of their proposal practice ............................................. 74

10. Ontological continuum positioned in a science and engineering culture ........................ 116

11. Textural and graphical viewpoint on the ontological continuum positioned in a

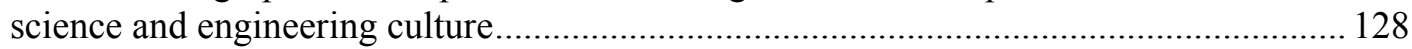

12. Proposal practice group dynamics. Note, there is no adjoining stage ............................. 148

13. Proposal community of practice graphical conditional matrix ....................................... 155

14. Explicit dimensions of competency, capacity, and capability in the proposal

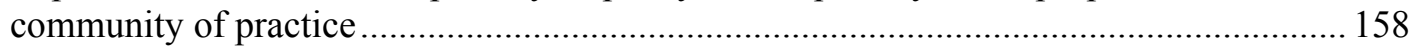

15. Proposal practice adapts and evolves to move along the ontological continuum.............. 160

16. Meta-inquiry discovers-owning, visioning, and contributing-and enhances the researcher's awareness of the proposal ecology allowing the emergence of substantive theory including reviewing and producing ................................................................. 172

\section{TABLES}

1. Grounded theory reflective coding matrix incorporating meta-inquiry............................ 30

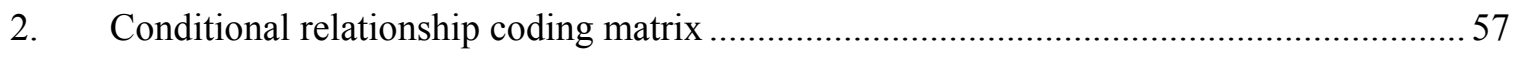

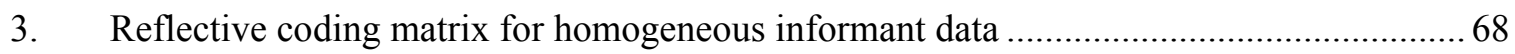

4. Reflective coding matrix - homogeneous informant and debrief data............................... 89

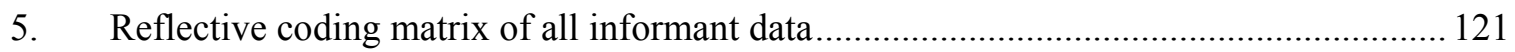

6. Partial conditional matrix for proposal community of practice ........................................ 145

7. Full conditional matrix for proposal community of practice........................................... 163

8. Explicit studies related to communities of practice in an organization ............................ 181 
Community of Practice xii 


\section{Chapter 1}

\section{Introduction}

Reflect on the times in your life when, with the assistance of others, you reached a personal best. Perhaps it was performing a piano recital, presenting a speech to a filled auditorium, or anchoring a relay team. With the encouragement and guidance of parents, teachers, peers, or teammates, you opened doors to your potential and radiated a sense of accomplishment. Initial fear turned to confidence, or at least increased comfort. How many of your personal bests thread to accomplishments at work?

Now, recall the feelings you experienced on the first major work assignment where you were responsible for the outcome. How honored you were at being selected by your supervisor. Compare your experience to my first grant writing opportunity. I was responsible for decoding the grant language, coordinating my thoughts and key strokes, soliciting input from others with varied success, spending hours doing financial calculations, struggling to format text and references, discovering all the features of the graphics package but the one I needed, and constantly being reminded of the pending deadline by my supervisor. I remember no sense of accomplishment, rather a sense of absolute relief, as I put the completed document in the mailing envelope!

Why was this organizational accomplishment different from a personal best? I used knowledge and action to achieve a measurable goal, but in the organizational setting, task completion was a conquest with no applause, a performance with no audience, a relay with one runner. Perhaps the learning culture that scaffolds our personal bests does not flourish in the workplace. But for organizations to thrive, employees' knowledge and accomplishments must be cultivated and leveraged to the strategic advantage of the company. 
For an organization to remain competitive, work processes must contribute to a culture of strategic learning (Cunningham, 1994). In the twenty-first century, technologybased organizations such as research laboratories and applied engineering firms employ highly educated employees. You might think that an organization comprised mainly of college educated workers guarantees a learning culture. But, traditional university training focuses on individual achievement, promoting competition among individuals; thus, the workforce must "learn" their true competition is other laboratories, agencies, and companies pursuing similar research funds or engineering contracts, not fellow team members (Cohen \& Levinthal, 1990; Duderstadt, 1999; Glaser, 1964; Merton, 1957). Figure 1 illustrates the required approach for an organization to achieve an edge over the true competition-converging business strategy, individual development, technology, market trends, skill mix, funding sources, and organizational culture to a single strategic focus, securing vital research and engineering funds (Cunningham, 1994; Hofstede, 1997; Pedler, Burgoyne, \& Boydell, 1997). To compete and excel, the workforce needs to

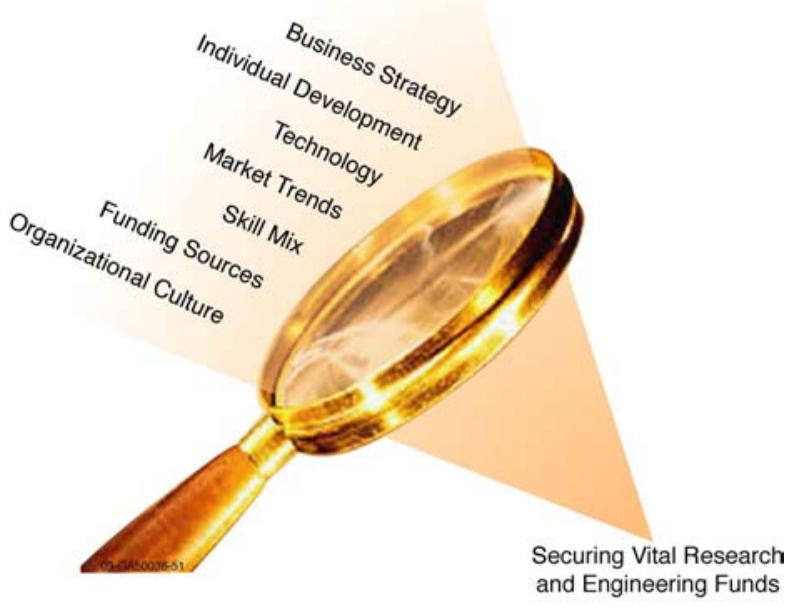

Figure 1. Strategic organization focuses critical processes achieving a competitive advantage. 
function as a strategic learning community, to proactively advocate the need for and value of new scientific and engineering capabilities (Storck \& Hill, 2000). That learning community must include key members of a transformative action team including research and engineering managers, scientists, engineers, and technical communicators, as well as finance, strategic planning, and business management specialists (Margolis \& Bell, 1989).

Generating a list of competencies is sometimes viewed as the initial step in the strategic learning process. Cataloguing competencies captures an organization's past rather than its future and promotes comfort in the status quo, especially when organizational development strategies reinforce the current or historical company processes (Argyris, 1993; Pedler, Burgoyne, \& Boydell, 1997). Rather an organization must build absorptive capacity, the ability to recognize the value of new information, to bridge existing competencies, and to assimilate new knowledge necessary to develop capability (Cohen \& Levinthal, 1990; Dixon, 2000). The holistic concept of capability, "the all-round human quality, an integration of skills, knowledge, and personal qualities used appropriately and effectively in a variety of familiar and unfamiliar situations" (Cunningham, 1994, p. 85), focuses strategies on the future. To achieve success, the organization must move beyond average and excel, and it must be recognized and rewarded for that excellence both nationally and internationally by receiving research funds and winning engineering contracts. To assist in securing new funds, the proposal process must move beyond a collection of competent individuals working independently to an integrated, interdependent team process that blends competency, absorptive capacity, and capability to the strategic advantage of the organization. 
The highly competitive and risk laden activity of proposal writing in science and engineering fields requires the teaming of managers, scientists, engineers, and technical communicators to conformally and responsively address a request for proposal (Friedland $\&$ Folt, 2000). The proposal must be persuasive and "convince readers not only that the work will be valid and important but also that they should pay for it!" (Penrose \& Katz, 1998, p. 116). Thus for the proposal team, managers, and authors to achieve success and develop absorptive capacity, they must move beyond building the individual competencies of selected team members and assigning arbitrary value to each team member's contribution and focus on the strategic goal of securing new funds.

The developmental and apprenticeship perspectives of adult education are integral in the process of developing absorptive capacity (Carlson, May, Loertscher, \& Cobia, 2003; Capezio, 1996; Donoghue, Harris, \& Weitzman, 1999; Johnson \& Pratt, 1998; Lave \& Wenger, 1991; McDonough, 2000; Pratt, 1998). From the developmental perspective, the knowledge, intellect, and ability to bring about transformative, strategic learning resides within the current workforce. In the apprenticeship perspective the learner guided by a mentor integrates essential explicit and tacit process knowledge through action resulting in acceptable practice. For optimum learning, the mentor and apprentice need to have proximity, work close to each other, and synchronicity, perform tasks together (Hughes, 2002).

In both perspectives open communication, dialogue, is critical to develop absorptive capacity. But in many organizations, communication is instrumental in nature, designed to get others to agree or to accomplish a goal rather than build new knowledge. Through dialogue, employees address the issues of why or the ends the organization serves. In dialogue, they continually discuss and relate the organization's purpose to the 
organization's mission, vision, and critical outcomes. Employees must be cognizant of the goal, or purpose, their work serves. If they observe practices that do not support the goal, they must challenge the practice by engaging in public dialogue (Dixon, 1998).

\section{Problem Statement}

The problem addressed by this research explores essential communication tools and processes needed to cultivate high functioning cross-disciplinary teams engaged in proposal preparation. Cross-disciplinary proposal teams blend the positivist view of the scientist with the constructivist view of the engineer, manager, editor, graphic artist, and text processor (Hughes, 2002). The need for cross-disciplinary proposal teams draws on personal data collected in the summer of 1999 as well as unpublished organizational needs assessment data collected between 1994 and 1999.

To obtain useful information, the assessment process must use dialogue and reflection to surface issues relating to the organization's purpose and approach to accomplishing goals and avoid manipulating the process to achieve a predetermined end (Bennett, Fadil, \& Greenwood, 1994; Dixon, 1998). In 1999, I interviewed one researcher from each of two previously completed needs assessment teams and established that generative listening and analysis processes occurred during informant interviews. The generative process includes suspending judgment while listening, talking of the group as a whole, suspending advice giving and problem solving, and reflecting. As a third needs assessment, I arranged individual interviews with a focused group of managers. The managers interviewed facilitate scientific and engineering groups actively involved in cutting edge research, nationally recognized for contributing knowledge to the science and engineering community through publications, and successfully competing for research funds from a variety of agencies. Because of the presence of these success 
indicators, I assumed the managers and their groups possessed some level of absorptive capacity and had developed the capability to successfully deal with and flourish in an increasingly competitive market for securing new funds. These data formed the basis for identifying enabling proposal processes that could result in measurable improvements in the proposal win rate. Dixon (1990) captures the importance of a well-designed, organizationally linked process when she states "one lesson...[we] can learn from manufacturing is that unless the measurement tools are sophisticated enough to detect error, the error will not be corrected" (p. 166).

Evaluation of data from the three needs assessments established the potential to increase proposal win rates by focusing on management identified critical proposals, by allowing authors to focus on technical and scientific content, and by providing a proposal process framework that includes assistance with editing, graphics, technical review, text processing, and printing. I assembled a core group of editors with a history of moderate success in supporting proposals and developed a proposal process aligned with laboratory writing practices (Bennett, Fadil, \& Greenwood, 1994). The proposal process flow is shown in Figure 2. The quantitative results of the proposal team's success in assisting scientists' and engineers' proposal efforts appear in reports published for fiscal years 1999, 2000, and 2001 (Carlson, 2001; Carlson, 2002; Carlson, Kaplan, and Einerson, 2000). Because of the submittal-award process, winning proposals prepared in 1999 were awarded funds in 2000 . The proposal team has assisted in receipt of $\$ 6.5 \mathrm{M}, \$ 9.5 \mathrm{M}$, and $\$ 30 \mathrm{M}$ of new funds in fiscal years 2000,2001 , and 2002, respectively. 


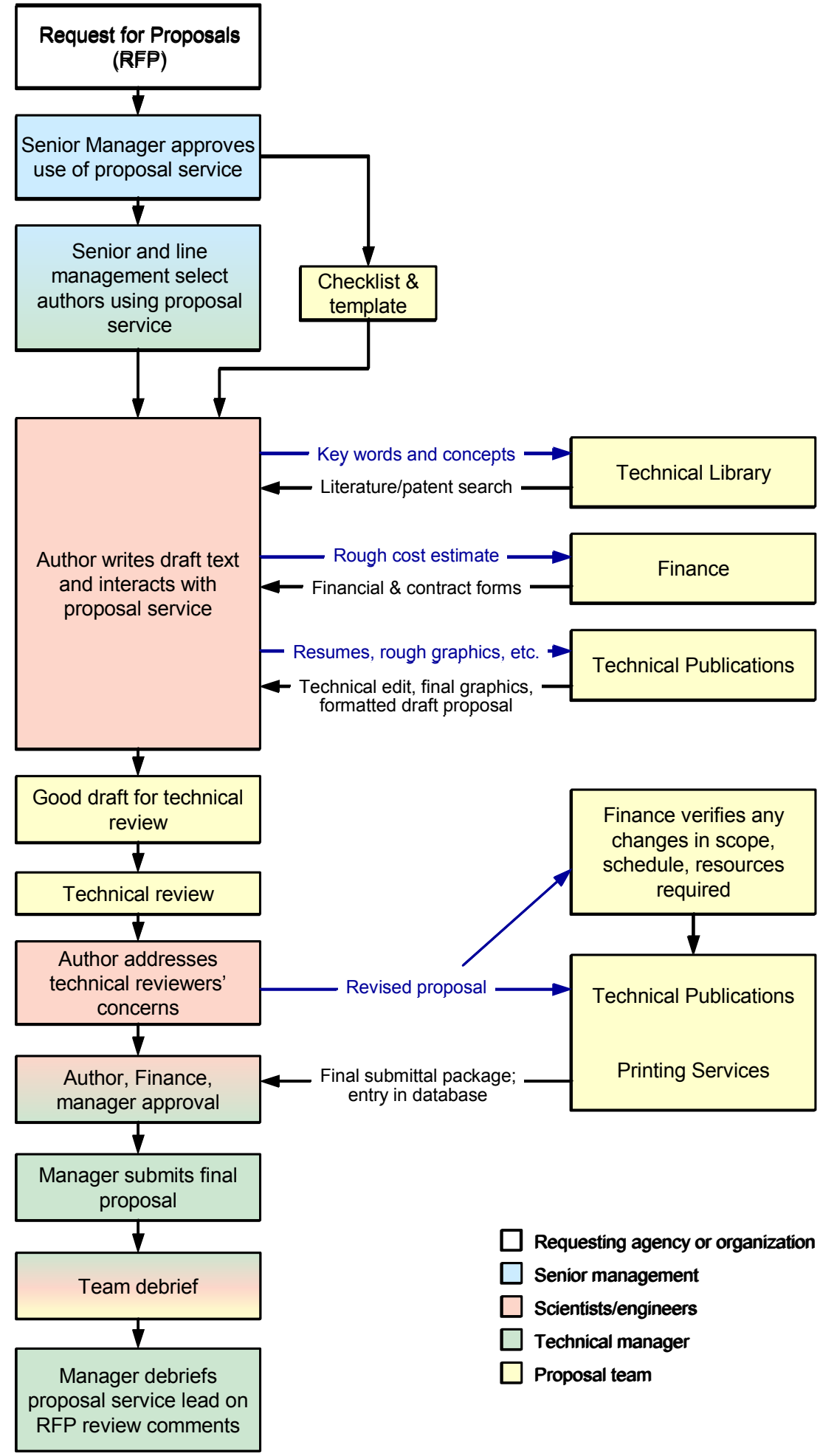

Figure 2. Work flow of the current proposal process. 


\section{Purpose of Study}

Although quantitative measures track and account for new funds to the laboratory, these measures do not describe proposal team member attributes critical for developing a synergistic team to assist authors in the development of a competitive proposal. Nor do the measures describe the proposal process tools needed by proposal team members, authors, and technical reviewers to facilitate the preparation of a conformal, responsive proposal. These concerns connect the core purposes of the research-

Describe essential team member attributes of a cross-disciplinary proposal team assisting authors in the development of competitive proposals.

Discover proposal tools that assist and are valued by a cross-disciplinary proposal team developing conformal, responsive proposals on a time-constrained schedule.

To discover the attributes of team members and the essential tools needed to prepare proposals, qualitative methodologies are well suited. I blend two qualitative traditions, ethnography and grounded theory, to achieve the stated research purpose. Ethnography provides "a description and interpretation of a cultural or social group or system" (Creswell, 1998, p. 58). Traditionally, ethnographic research "involves prolonged observation of the group, typically through participant observation in which the researcher is immersed" (Creswell, 1998, p. 58) in the culture. Lawrence-Lightfoot and Davis (1997) add two additional dimensions to Creswell's definition of participant observation-the reality of experiences observed in natural settings and researcher selfmonitoring by active reflection and continual questioning.

My research uses a facet of the ethnographic tradition, topic-oriented ethnography, to focus on an aspect of organizational life, proposal preparation, existing in the work community (Spradley, 1980). Topic-oriented ethnography captures three 
primary elements of the proposal community-place, participants, and activities. Through topic-oriented ethnography, the knowledge gained about the unique cultures of participants enables me to actively frame and value each informant's contributions to the proposal process. The ethnographic data also enhance grounded theory analysis and interpretation as informant voices can be more clearly understood.

Researchers in grounded theory and ethnography discuss the interrelatedness of the two qualitative methodologies. Glaser and Strauss (1967) present ethnography as a companion to grounded theory. "Ethnographic studies and multiple theories are needed so that various substantive and formal areas of inquiry can continue to build up to more inclusive formal theories" (p. 35). And Spradley (1980) points to the connectedness of ethnography to grounded theory when he states,

Much social science research has been directed toward the task of testing formal theories. One alternative to such theories, and a strategy that reduces ethnocentrism, is the development of theories grounded in empirical data of cultural description, what Glaser and Strauss (1967) have called "grounded theory". Ethnography offers an excellent strategy for discovering grounded theory. (p. 15)

Delimitations and Limitations

\section{Delimitations}

I narrowed the scope of the research by initially focusing on informants involved in an organized proposal process in a research and applied engineering laboratory. A further narrowing of the scope of the research results from the alignment of team proposal efforts with only those funding opportunities considered critical to the mission 
of the laboratory by senior managers. The funding efforts link to the nation's energy, security, environmental, and science needs and frame the place, participants, and activities of the research. The proposal process provides a social setting in which participant observations and dialogue can occur within the normal flow of the proposal process; thus, the ethnographic criteria of simplicity, accessibility, unobtrusiveness, permissibility, and frequency of recurring activity are possible to achieve in this study (Spradley, 1980).

\section{Limitations}

The place, participants, and activities also bound the research as the proposal process responds only to solicitations directly linked to the nation's energy, security, environmental, and science needs. The research is also culturally bound to the selected research and applied engineering laboratory setting (Kaplan, 1999). Emergent theory from this singular research setting should benefit other organizations responding to proposal requests, but the theory may need to be modified. The need for modification of the theory could be viewed as a research limitation, but modification is an inherent strength of grounded theory as modification results in greater transferability and generalizability of the emergent theory (Tashakkori \& Teddlie, 1998).

The proposal team focuses only on preparation of documents responding to prescriptive solicitations or developing critical funding documents conforming to established laboratory guidelines and regulatory agencies expectations. Also, the proposal process must produce conformal and responsive proposals while adhering to time sensitive submittal dates established by external agencies or organizations. Quality documents are usually produced in 30 days or less from the time proposal team assistance is requested. 


\section{Definitions}

Due to the inductive nature of both ethnography and grounded theory, terms evolved as the data are collected and the extant literature reviewed. I used the convention suggested by Creswell (1994) of underlining the term then providing the definition in paragraph form.

Attribute: “Any element of information regularly associated with a cultural category" (Spradley, 1980, p. 131). For the research, discipline culture-linked attributes as well as the common team attributes essential for proposal team success are discovered and linked (Beane, 2000).

Community of practice: Wenger (1998) defines a community of practice as both a community and an economy enterprise. "A joint enterprise brings the community together through the collective development of a shared practice... negotiated among the participants through...the politics of participation and reification" (p. 209). A community's shared practice produces artifacts valued by others. Communities of practice are not a community of interest or a geographical community as neither of these communities implies a shared practice resulting in artifacts of value (Wenger, 2000a).

Culture: Creswell (1998) provides the definition of culture used in this research. Culture "consists of looking for what people do (behaviors), what they say (language), and some tension between what they really do and what they ought to do as well as what they make and use (artifacts)" (p. 59).

Discipline: "A body of theory and technique that must be studied and mastered to be put into practice. A discipline is a developmental path for acquiring certain skills or competencies" (Senge, 1990, p. 10). 
Grounded theory: A qualitative methodology that discovers theory from data systematically obtained, analyzed, and linked to a basic social process (Glaser, 1978).

Language: Managers, scientists, engineers, and writers communicate in their own unique, discipline jargon, a technical subset of the English language (Bennett, Fadil, \& Greenwood, 1994; McDonough, 2000). The discovery of key language components and the unique meanings of those components is crucial for proposal success. The term, funding, illustrates a difference in interpretation by participants based in their discipline languages. For the manager, funding can center on an awarded dollar amount that is summed with other dollars to achieve a sales target. For the scientist or engineer, funding translates into doing self-defined research for a set duration of time. The proposed research is deemed meritorious by an external review panel of peers who concur that the research has the potential to add to the volume of science knowledge. For the editor, graphic artist, and text processor, there is only an indirect connection between funding and their sense of job security, as they do not participate actively in the funded research.

Participant: "Someone who becomes the object of observation in a natural setting” (Spradley, 1979, p. 32). All participants come from unique communities, or discipline cultures-managers, scientists, engineers, and the technical publications staffeditors, graphic artists, and text processors. Each community has a unique culture, language, perspective, and goal orientation-making proposal writing a strategic learning process with many dynamics.

Place: The focus of the study is a research and applied engineering laboratory. Topic-oriented ethnography: This facet of the ethnographic tradition focuses on "one or more aspects of life known to exist in the community" (Spradley, 1980, p. 31). 
For this study, the aspect of organizational life described is a community of practice involved in the proposal process.

Meta-inquiry: An addition to grounded theory methodology that involves the coding, interpretation, and valuing of the responses garnered through initial interviews from a small homogeneous informant group. The grand tour question is articulated following meta-inquiry (Carlson \& McCaslin, in press).

\section{Significance of the Research}

Organizational culture is learned. "The organization establishes routines for conducting work that reflects organizational style, safety culture, and communication" (Blackman \& Gertman, 2002, p. 3). Thus, theory for enhancing the effectiveness of a cross-disciplinary proposal team in developing competitive proposals discovered from this research could be incorporated into the laboratory's organizational culture. "People everywhere learn their culture by observing other people, listening to them, and then making inferences" (Spradley, 1979, p. 8). Just as the organization learns to use an ineffective process, observing a successful process should result in a desire to participate in or imitate the process.

For the proposal process to succeed, different disciplines must work synergistically to create an artifact of value, a winning proposal. By observing and discovering the cultural dynamics of the process, the contribution of each participant's discipline-linked culture is more clearly understood and valued. Because of the crossdisciplinary aspects of the proposal process, the research provides insight into an organizational process occurring at cultural boundaries of scientists, engineers, managers, and the proposal team practice. Discovering boundary activity dynamics and establishing the value of those dynamics in the creation of strategic knowledge provides insight to 
move a competency-limited organization to a capability-driven organization through the process of building absorptive capacity (Cunningham, 1994; Robey, Khoo, \& Powers, 2000). Rather than providing catch phrases or team building ideas, the research initially provides a substantive theory grounded in the proposal process that explains what enables the team to produce artifacts that measurably benefit the future of the organization.

Emergent formal theory about cross-disciplinary teams working on timeconstrained tasks is not limited to organizations developing proposals. Similar processes occur in a hospital emergency room when treating a trauma patient, during a relief effort following a disaster, in the control room of a nuclear reactor during an off-normal event, or during a factory shutdown to replace a critical component. In all these situations, participants with different disciplines must work together to achieve a critical, timeconstrained goal. The research provides a theoretical approach to establish the attributes of team members well suited for joint enterprise in a strategic community of practice as well as the tools each member needs to participate at their full potential within the community. 


\section{Chapter 2}

\section{Initial Literature Review}

The initial literature review focused on fields peripherally related to the problem statement following the methodological approach to grounded theory explained by Glaser (1992).

When the theory seems sufficiently grounded in a core variable and in an emerging integration of categories and properties, then the researcher may begin to review the literature in the substantive field and relate the literature to his own work in many ways. (p. 32)

Strauss and Corbin (1990) provide additional insight relating to the need for an initial visit of the literature to establish theoretical conditioning through gaining insight into research work pertinent to the study. Their approach stresses awareness of the activities within the human ecology as it relates to the anticipated informant data, and shows less concern than Glaser does about exploring existing hypotheses.

Initially, I explored several bodies of knowledge as part of my theoretical conditioning. Using the extant literature, the major areas reviewed included qualitative methodologies (Creswell, 1994, 1998), ethnography (Denzin, 1997; Spradley, 1979, 1980), grounded theory (Glaser, 1978, 1992, 2001; Glaser \& Strauss, 1967; Strauss \& Corbin, 1990), group dynamics (Forsyth, 1999), interviewing strategies (Rubin \& Rubin, 1995), leadership (McCaslin, 1995, 1996), individual and organizational development (Argyris, 1986; Collard, 1998; Covey, 1989; Robinson \& Robinson, 1990), organizational cultures (Glaser 1964; Hofstede, 1997), group communication strategies (Dixon, 1998, 2000), communities of practice (Lave \& Wenger, 1991; Wenger, 1998, 1999, 2000a, 2000b; Wenger \& Snyder, 2002), adult learning perspectives (Palmer, 1998; 
Pratt, 1998), strategic organizational learning (Blackman \& Gertman, 2002; Cohen \& Levinthal, 1990; Cunningham, 1994; Donoghue, Harris, \& Weitzman, 1999; Pedler \& Aspinall, 1998; Pedler, Burgoyne, \& Boydell, 1997; Storck \& Hill, 2000) and effective proposal writing strategies (Friedland \& Folt, 2000; Penrose \& Katz, 1998). To minimize researcher theoretical bias to theories directly related to the research problem, I did not read any literature specifically addressing cross-disciplinary teams and time-constrained work processes thus "allowing substantive concepts and hypotheses to emerge first, on their own" (Glaser \& Strauss, 1967, p. 34). My substantive theory emerges from the voices of the informants engaged in the proposal process.

Some of the reviewed literature provided general concepts for self-improvement (Covey, 1989), approaches to organizational development (Argyris, 1986; Collard, 1998; Robinson \& Robinson, 1990), and offerings of countless seminars each year on proposal and grant writing. Individual learning can improve self-image, build team skills, and improve writing skills, but the learning occurs as unrelated packets of knowledge without an integrative strategy to connect and leverage that knowledge to the strategic benefit of an organization.

For strategic organizational learning and growth to occur, an organization must develop enabling basic social processes such as interdependence (Glaser, 1978). Interdependence, or collectivism, is the norm in eastern culture while individualism is the norm in western culture (Forsyth, 1999; Hofstede, 1997). Hofstede (1997) provides the following insight into the cultural implications of individualism and collectivism when he writes,

Individualism pertains to societies in which the ties between individuals are loose: everyone is expected to look after himself or herself and his or 
her immediate family. Collectivism as its opposite pertains to societies in which people from birth onwards are integrated into strong, cohesive ingroups, which throughout people's lifetime continue to protect them in exchange for unquestioning loyalty. (p. 51)

The proposal service positioned in a fiercely independent, western, science and engineering culture, faces unique strategic learning challenges and opportunities to function as an interdependent community of practice (Glaser, 1964; Merton, 1957; Polanyi, 1983).

\section{Forming a Strategic Learning Community}

Communities of practice literature provides insight into the basic social processes of participants engaged in common practice and promotes connected learning to integrate explicit and tacit knowledge (Dixon, 2000). A community of practice is a social rather than an individual process where content is learned in the context of joint enterprise, called practice. A community of practice results in the generation of knowledge through social participation. Learning results by engaging in social practice to negotiate meaning, by using imagination to discover spatial and temporal connections, and by aligning activities to enhance the enterprise (Wenger, 1998). In this social setting, knowledge is used, reused, negotiated, defined, shaped, connected, stewarded, and valued by community of practice members; thus, learning is a developmental interplay between "social competence and personal experience" (Wenger, 2000b, p. 225).

A community of practice is an informal social structure that has no charter, manager, or executive officer. Shared expertise and passion for practice connect members. A community of practice's purpose is to develop knowledge, not to allocate resources or manage people (Wenger, 2000a). Thus the research into the proposal team 
has the potential to provide organizations with ways to foster communities of practice linked with strategic business processes.

There are various levels of participation within a community of practice, as shown in Figure 3 (Wenger, 2000a). The core group is a small, energizing group called masters who establish ideals, enhance practice standards, and are highly respected by the community. Full membership describes members possessing the explicit and tacit knowledge needed to practice within the community. They are often called upon to function in the role of mentors to those new to the practice. Peripheral membership belongs to newcomers, the apprentices, with casual practice in the community's body of knowledge. Transactional participation describes outsiders who occasionally interact with the community or provide a needed service. Passive access includes people who value artifacts produced by the community such as websites, publications, standards, recordings, or art objects. The trajectory to the core of the community involves moving from low- to high-risk procedures and from simple to complex understanding as the learner develops higher-order thinking and problem solving skills (Carnoy, 2000; Dirkx \& Prenger, 1997; McDaniel, Schmidt, \& Hunter, 1988; Pratt, 1998; Wenger, 1999).

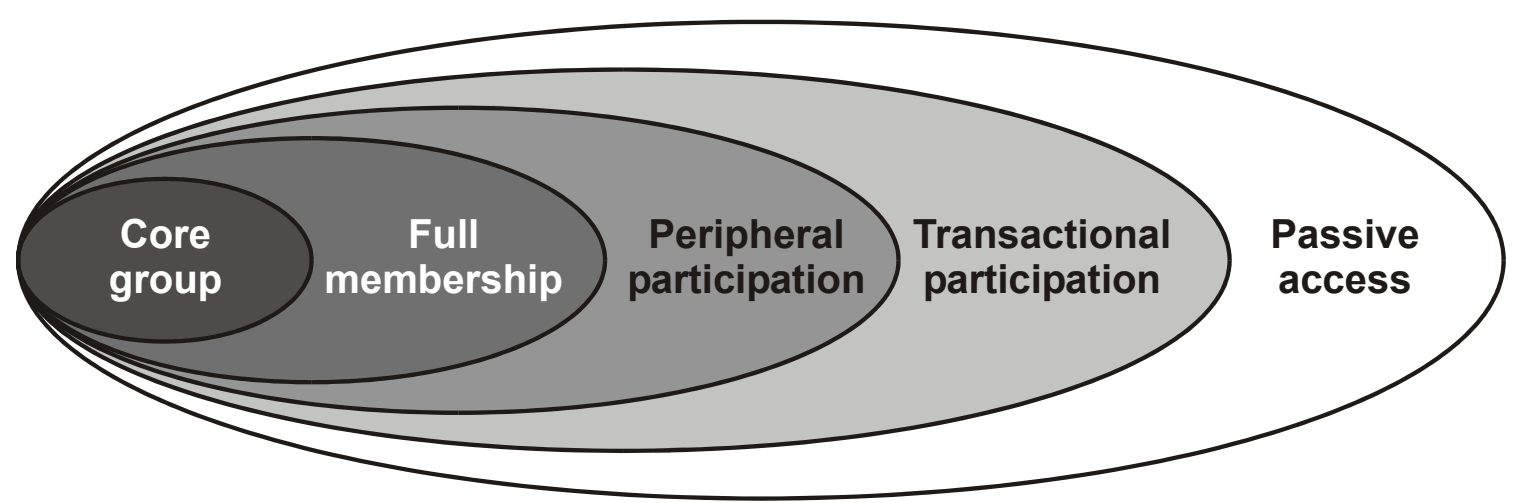

Figure 3. Levels of participation in a community of practice (Wenger, 2000a). 
The knowledge and expertise of a community of practice reside within the group rather than within any one individual. The community's ideals are established collectively by the masters and transferred to apprentices by mentors. The apprentice learns through practice with the guidance of a mentor who facilitates the apprentice's enculturation process by guiding their understanding of their role and identity while mastering skills in the workplace (Pratt, 1998). The learner acquires both explicit and tacit knowledge framed in the context of practice resulting in a learning experience actively linked with the application of transferred knowledge. A community of practice strives to ensure the success of its members (Wenger, 2000a). Failure to succeed at a job is most often linked to a lack of understanding of the community of practice rather than a lack of knowledge of the profession.

Traditional apprenticeships, based in the skilled trades, assume the newcomer has little formal academic training. By exposing the learner to the potential of the profession, the mentor assists the learner to expand their active participation in their community of practice (Bennett, Fadil, \& Greenwood, 1994; Dirkx \& Prenger, 1997; Parks Daloz, 1990; Wenger, 2000b). A modern version of the Middle Age apprenticeship concept is the internship. The intern comes to the community of practice with academic knowledge, and the mentors provide a scaffolded process to connect current academic content in the context of a work process (Premont, 1990). The proposal practice aligns with the concept of an internship, as each participant comes to the community with knowledge in a specific discipline.

In the proposal process, the technical publication team members represent the core group. The team negotiates and renegotiates the knowledge and tools needed to assist authors to successfully navigate the time-constrained proposal preparation process. 
Although the core group does not possess the unique scientific or engineering concepts that form the backbone of a proposal, they possess knowledge about how to assist in crafting a persuasive document through the use of graphics and text. Thus, the core members assist extremely bright scientists and engineers to navigate the process steps of proposal writing and production. The scientists and engineers though extremely capable in their chosen discipline may be newcomers to the proposal process. Depending on their level of proposal writing knowledge, authors may span an author-skill gradient from peripheral participant to core member. The technical publications staff must discern the level of assistance required and counsel, coach, or mentor based on the author's knowledge of the proposal preparation process (National Press Publications, 2001). 


\section{Chapter 3}

\section{Assumptions and Rationale for the Use of Ethnography and Grounded}

\section{Theory}

Because several discipline cultures combine to prepare a proposal, the interview process springs from the ethnographic tradition. Cultural awareness of informants helps me frame their responses during dialogue and weave their voices through grounded theory analysis and interpretation. To prepare for dialoguing with informants, I needed to discover an effective interview strategy and develop culturally meaningful questions because "if the questions one asks are not crucial, then differences in responses are not crucial either" (Wolcott, 1998, p. 325). Anfara, Brown and Mangione (2002) echo the importance of the interview strategy when they say, "Keeping in mind that research questions provide the scaffolding for the investigation and the cornerstone for the analysis of the data, researchers should form interview questions on the basis of what truly needs to be known" (p. 31).

To frame my qualitative research approach in the culture of the study, I used an initial interview protocol with a homogeneous group of informants, the proposal service team, followed by a preanalysis process, called meta-inquiry, before developing a formal interview protocol and the grand tour question. Meta-inquiry helps make the grounded theory discovery process explicit with a culturally focused grand tour question (Anfara, Brown, \& Mangione, 2002). The interview approach is shown in Figure 4. Two new grounded theory dimensions, theoretical conditioning and selective questioning, precede meta-inquiry (Carlson \& McCaslin, in press). 
During theoretical conditioning, the researcher explores key literature and spends time understanding the human ecology of the study. My initial academic training focused on the area of field ecology. The biological concept of ecology is well suited for

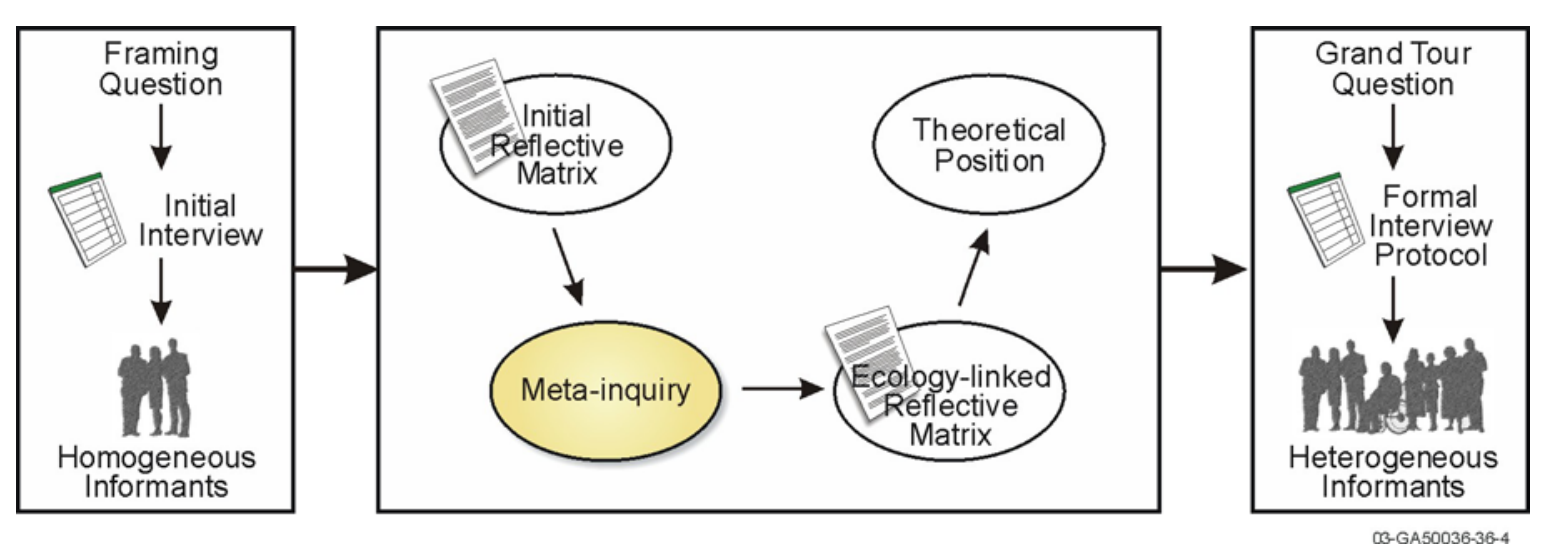

Figure 4. The position of meta-inquiry in the interview process.

qualitative research in human interactions, a human ecology. Odum (1959) addressees the relationship of animals to their biotic community and explains

Organisms do not live alone, nor do they ever have an entirely private autoecology. Organisms living in any given area, whether large or small, are associated in what is known as biotic communities. The biotic community is rather loosely but definitely held together as a unit by the interdependence of its members. (p. 145)

Also, the human ecology, a unique biotic community, is composed of smaller groups. "The members of which are more intimately associated with each other, and hence these groups form a cohesive unit. Such units within the community are called populations" (Odum, 1959, p. 145). 
The relationship of the biotic communities, called communities of practice in this research, and the populations, the informants' unique discipline cultures, parallels a human ecology. This parallel stresses

That the population and community are real entities, even though one cannot usually pick them up and put them in a collecting kit as one would collect an organism. They are real things, because these group units have characteristics additional to the characteristics of the individuals composing them. (Odum, 1959, p. 146)

Odum (1959) also advocates that to gain a better understanding of an ecosystem, one should study a rare species to more clearly appreciate the interdependencies in the biotic community. The proposal process community of practice weaves and interconnects the characteristics of unique populations composed of editors, graphic artists, text processors, production staff, scientists, engineers, and managers interacting in a research and applied engineering laboratory-a human ecology.

McIsaac and Morey (1998) situate the human ecology from the engineer and scientist's perspective when they discuss the role of deduction and induction in scientific discovery.

Within science, there have been two divergent approaches to discovery: deductive and inductive. The deductive method, on the one hand, seems to fit well in physics, mathematics, and the traditional culture of engineering science, because deduction follows a logical, systematic, and orderly progression. Induction, on the other hand, depends on creative insight into observed phenomena. Although intuitive insight plays a role in the physical sciences, induction seems to be relatively more important in the 
study and management of highly complex and interactive systems, such as in ecosystems and social systems. (p. 113)

With the heightened awareness of the human ecology gained through theoretical conditioning, I developed culturally linked interview questions using selective questioning. Selective questioning examines areas to explore in the initial interviews based on knowledge and reflective sorting. The researcher uses knowledge sorting to connect literature findings about place, participants, and activities to generate cultural understanding and prepare the researcher to examine key concepts, phenomena, and events that relate to the research. The reflective sorting process, which follows knowledge sorting, provides a triangulation and verification framework for use when constructing meaning from emerging substantive or formal theory (McCaslin, Scott, \& Carlson, 2002).

\section{$\underline{\text { Homogeneous Informant Interview Process }}$}

To start the research journey, I developed a framing question and main questions for the initial interviews with the core team-the editors, graphic artists, and text processors engaged in the proposal process. Using theoretical conditioning and selective questioning, I developed a framing question for the initial interview process with the homogeneous informant pool. The framing question recognizes and values the interrelatedness of the ecology and the interdependence of informants.

These questions were discovered and framed after nearly three years of negotiating knowledge with the core team. Some members interviewed have been with the proposal team for the entire three years, two moved to other positions based on their capabilities in handling complex processes, and three had interacted with the core team for less than six months at the time of their interviews. Thus the data collected from the 
core team, the homogeneous informant pool, provide a range of temporal and spatial perspectives on the proposal process.

The framing question is as follows:

What impact can cross-disciplinary team processes have on strategic organizational learning?

Using five initial interview questions, I collected data from each core team member.

- What attributes are important for a member to possess to contribute to a proposal effort and why?

- What proposal service developed tools do you most frequently use and why?

- What features of the tools are most helpful? Which are least helpful?

- $\quad$ Based on your interactions with proposal service customers, what do they value most about the service? What do they find least beneficial?

- What personal value do you place on working with the proposal service?

Although these were the initial main questions, the question-answer process followed the ethnographic tradition. Spradley (1980) provides insight into the process when he writes:

Ethnography begins with a different assumption: that the question-answer sequence is a single element in human thinking. Questions always imply answers. Statements of any kind always imply questions. This is true even when the questions or answers remain unstated. In doing participant observation for ethnographic purposes, as far as possible, both questions and answers must be discovered in the social situation being studied. (p. 32) 
The interview data from the core team members were recorded and transcribed along with my journal entries. Meta-inquiry, the coding, interpretation, and valuing of the initial responses garnered from a small homogeneous informant group through initial interviews, followed each interview. Meta-inquiry adds an essential step to grounded theory by reconciling initial interview data acquired from a homogenous group of informants and deepening my understanding of the human ecology (Carlson \& McCaslin, in press).

The human ecology is discovered through the use of topic-oriented ethnography. Topic-oriented ethnography is an essential and necessary component of meta-inquiry allowing the researcher to gain a rich understanding of the interdependencies and dynamics of the human ecology. Meta-inquiry of initial interview data allowed me to assess, modify, enhance, and focus the formal interview protocol based on data from a homogeneous pool of informants. Using topic-oriented ethnography, I gained a greater appreciation of informants' discipline cultures in the proposal ecology thus preparing me for meta-inquiry. This grounding in the discipline cultures scaffolded the acquisition of data from which concepts, categories, properties, processes, dimensions, and theory emerge during grounded theory analysis and interpretation of informant data.

The result of meta-inquiry is a theoretical position from which the grand tour question emerges; a grand tour question grounded in the research culture. Figure 5 shows the positioning of meta-inquiry and the grand tour question in the grounded theory methodology.

To illustrate the efficacy of understanding the human ecology when framing the grand tour question, Table 1 shows the result of meta-inquiry's application in the analysis of data from a small research study involving a homogeneous informant pool of adult 
education graduate students actively involved in graduate course work. The framing question used during initial interviews was - "How are dissertation topics discovered, developed, and chosen?" Following coding and meta-inquiry, insight was gained into a more appropriate grand tour question: "What guides and signposts direct a graduate 


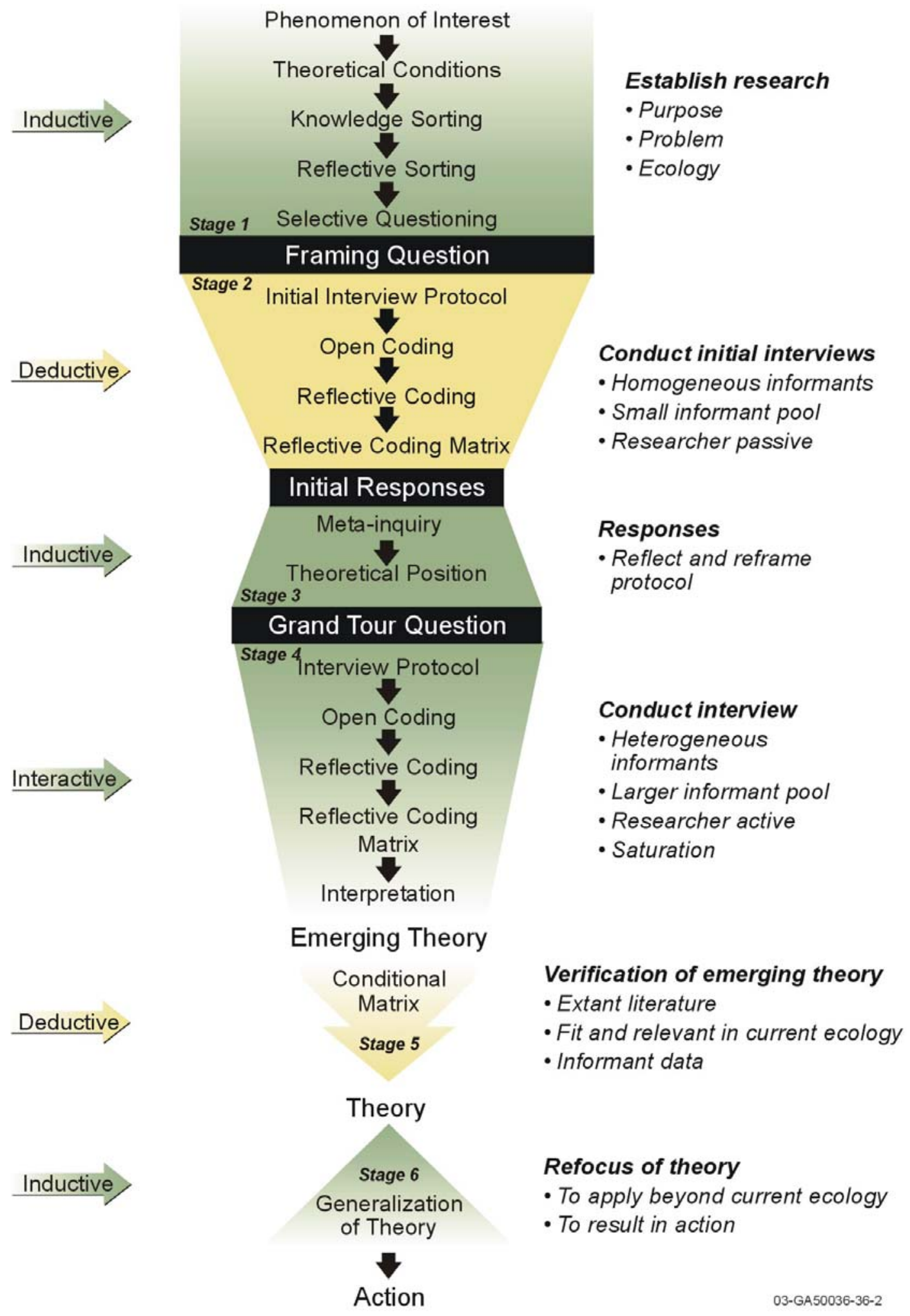

Figure 5. The six stages of grounded theory including meta-inquiry. 
Community of Practice 29 
Table 1

Grounded theory reflective coding matrix incorporating meta-inquiry

\begin{tabular}{|c|c|c|}
\hline \multicolumn{3}{|c|}{ Reflective Coding Matrix } \\
\hline Category Scaffolding & Motivat & nal Influences \\
\hline Properties & Le & el of Risk \\
\hline Process & Challenge - High Risk & Comfort - Low Risk \\
\hline$\underline{\text { imensions }}$ & $\begin{array}{l}\text { No topic selected yet; Challenging (mentioned by } 3 \text { informants); } \\
\text { level of expertise-novice; uncharted territory (mentioned by } 2 \\
\text { informants); Wants to teach in higher education; Teach nurses what } \\
\text { makes or identifies caring behavior; qualitative product that can be } \\
\text { beneficial to anyone or service providers; Formulating a theoretical } \\
\text { approach; Write a book (mentioned by } 2 \text { informants); Formulating } \\
\text { research goals; Theory leads to accreditation model; Catalyst for } \\
\text { further research; Spawns further research on subject of couples; } \\
\text { Justify funding; *Topic needs direction; Concerned that the } \\
\text { discipline isn't refined (wants more in-depth knowledge and } \\
\text { enlightenment); Discipline needs direction; Work on improving } \\
\text { integrity of discipline; Developing a hypothesis; Has not taken } \\
\text { preliminary exams; Exploration phase-several topics in mind; } \\
\text { Discipline is greatly needed in society }\end{array}$ & $\begin{array}{l}\text { Passion for topic; Insider connection to topic; Topic gestational based on } \\
\text { experience with labor and delivery; Challenge of the topic-Close to } \\
\text { information; Wants to be involved; Within comfort zone (mentioned by } 3 \\
\text { informants); Coaching level of expertise; Personal Connections (mentioned } \\
\text { by } 2 \text { informants); Related to subject; Personal experience in labor and } \\
\text { delivery; Life experiences are linked to topic; Topic is biographical; } \\
\text { Empathy for those in the study group; Cares for people in the study group } \\
\text { (mentioned by } 2 \text { informants); Study of marital relationships; Mother as } \\
\text { mentor; Professor as mentor; Caring mentor; Applied/previous hands on } \\
\text { experience (mentioned by } 2 \text { informants); Case studies; Expertise due to } \\
\text { exposure to people; Prior quantitative experience (mentioned by } 3 \\
\text { informants); Work experience; Studied other books on the topic; Examine } \\
\text { existing programs; Relates to thesis work; Undergraduate classes; Graduate } \\
\text { classes/degrees (mentioned by } 4 \text { informants); Past formal or higher }\end{array}$ \\
\hline
\end{tabular}




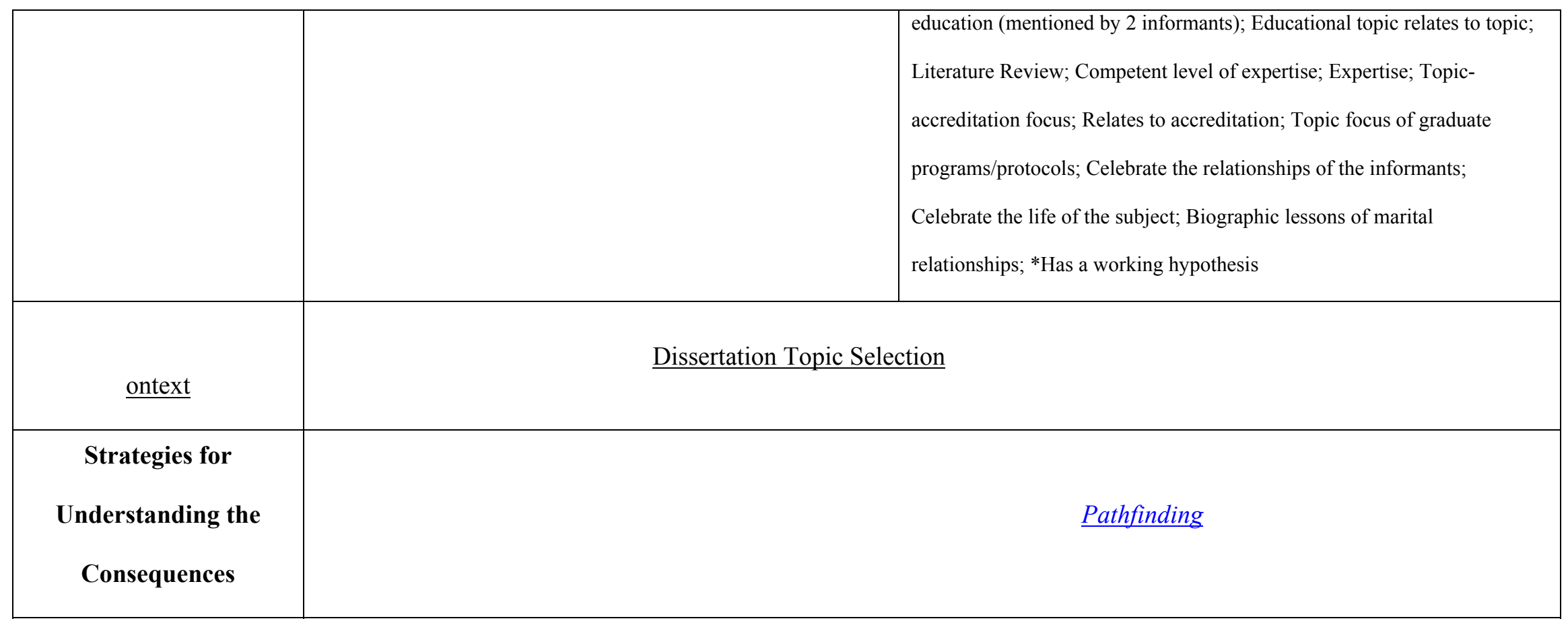


research journey?" (Carlson \& McCaslin, in press). This grand tour question can be posed to informants actively engaged in the doctoral process as well as informants currently or permanently withdrawn from the pursuit of a degree.

\section{$\underline{\text { Role of the Researcher }}$}

My research uses the traditions of ethnography and grounded theory to guide me in developing a greater understanding of the phenomenon of interest. Because I led the proposal service before I started the dissertation research, I have engaged in an informal meta-inquiry process for the past three years. The informal inquiry arose out of a need to actively dialogue with all team members and customers as part of the ongoing proposal process. The research provided me the opportunity to reflect on the current proposal process and to value each member's unique contribution.

Prior to establishing the proposal service, I had acquired over 30 years of knowledge in biology, chemistry, mathematics, and secondary and adult education and had spent 18 of those years as an active participant in scientific research focusing on the sensing and control of welding processes. During my time as a researcher, I was recognized as capable with an acceptable publication, presentation, and patent portfolio.

My dissertation research focuses on a substantive research area, the proposal process, in which I had limited academic discipline training. I explored the proposal process from the perspective of a scientist rather than a technical writer. Glaser (1978) suggests that

Switching substantive fields is vital. It generalizes the perspective of the analyst, which is exciting. It satisfies his curiosity for more knowledge of new areas. Most importantly, it derails the tracked thinking that normally occurs for an analyst when staying in the same substantive area. (p. 27) 
I am familiar with the discipline culture of the research scientist and the scientist's role in proposal writing. Since establishing the proposal service, I have interacted with scientists in disciplines different from mine, engineers working on applied engineering projects, managers of research, engineering, construction, and business processes, and a host of disciplines involved in technical publications. I came to realize that although we worked in the same laboratory, the cultures of these groups varied significantly. Additionally, the technical publication staff was not fully aware of the cultural diversity of their customer base and sometimes this lack of awareness resulted in document disasters and low customer satisfaction.

During my years as a research scientist, I observed processes that assisted me, hindered me, or did not exist. Thus, the proposal process resulted from a personally observed need to assist the scientific and engineering staff to bring new funds to the laboratory as well as an assessed need based on needs assessments previously described in Chapter 1. As an active participant in the proposal process in the role of the proposal service team leader, I have the unique opportunity to observe all participants, both as a provider of a service and as a performer of unique process steps.

Spradley (1980) states, “the highest level of involvement for ethnographers probably comes when they study a situation in which they are already ordinary participants" (p. 61). He offers the following caution to the researcher, who also assumes the role of an ordinary participant,

The more you know about a situation as an ordinary participant, the more difficult it is to study it as an ethnographer. It is no accident that ethnography was born and developed in the study of non-Western 
cultures. The less familiar you are with a social situation, the more you are able to see the tacit cultural rules at work. (p. 61-62)

To address Spradley's cautions, I maintained a daily log to capture my thoughts, reflections, observations, and feelings about daily proposal activities so that both explicit and tacit cultural knowledge could be captured and made explicit. The log captured my insights into informant data, provided a personal dialogue medium to enhance interview questions, assisted me during analysis, and provided a "parking lot" for emerging concepts and theory. In addition to the daily log, I involved another qualitative researcher, familiar with the human ecology of the study, to "provide an 'audit' trail of the key decisions made during the research process and validate that they were good decisions" (Creswell, 1994, p. 158).

Partial funding by the laboratory of my tuition, books, and fees introduced no researcher bias into my community of practice inquiry. The laboratory president and research director proactively linked me to other external proposal resources allowing me to investigate potential approaches for enhancing the laboratory's proposal process, but they made no requests or demands regarding a preferred proposal process. I engaged in active dialogue with the senior management team to validate that the proposal service aligned with the research direction of the laboratory. Additionally, I met with the director of research at least twice a year to update him on proposal service practices and successes. During these discussions the director provided insight into future research directions and upcoming funding opportunities, but did not advocate changes to the proposal practice. 


\section{Approach for Developing the Grand Tour Question}

Due to my heightened awareness of homogeneous informant culture achieved through meta-inquiry, I worked to increase my understanding by encouraging informants to describe their practice in their own terms and to provide in-depth details of their successes and concerns about the proposal process (Rubin \& Rubin, 1995). Thus, the grand tour question emerging from meta-inquiry is more culturally significant and moves my grounded theory research beyond the creation of a theoretical position, the result of meta-inquiry, towards a workable and testable theory truly grounded within a human ecology (McCaslin, 1996). Additionally, including meta-inquiry in grounded theory provides the reflective pause necessary to see the connections between initial informant data and the research environment and prepares me for the formal interview process with the heterogeneous informant pool. 


\section{Chapter 4}

\section{Meta-inquiry Data Analysis and Interpretation}

A qualitative research journey recognizes the interrelatedness of the human ecology, the informants, the problem statement, and the purpose of the research (McCaslin, 2002). Theoretical conditioning, knowledge and reflective sorting, and selective questioning allowed me to prepare an initial interview protocol based on a framing question and accompanying main questions focused on the proposal practice (Denzin, 1997; Rubin \& Rubin, 1995).

A theoretical position emerged from the analysis and interpretation of the core team interview data using two qualitative methodologies, ethnography and ground theory. Meta-inquiry provided a reflective pause before establishing the formal interview protocol used with a heterogeneous group of informants and resulted in the emergence of a theoretical position.

\section{$\underline{\text { Researcher Reflections }}$}

Informants freely shared their insights and reflections on their proposal practice and revealed the value of dialogue in a cross-disciplinary team. I discovered that many of my interactions with the team occurred on the transactional level and focused on document crafting and presentation (Bycio, Hackett, \& Allen, 1995; Forsyth, 1999; Howell \& Avolio, 1993). These transactional interactions, in part, result from the time critical nature of the proposal process and from my undervaluing the perspectives and insights of the team members about approaches to enhance the proposal practice.

The interviews provided the team with an opportunity to reflect, share, critique, and vision about the past, present, and future directions of the proposal practice. The interviews also provided me with an opportunity for self-reflection on strategies to 
re-craft my role in the proposal process. I need to enable team members by mentoring at the transformational level rather than managing at the transactional level (McCaslin, 1996; Rost, 1991). The team needs to be engaged in the evolution of their practice and proactively invest in their future proposal practice.

My current advocacy role with senior managers is critical for strategic alignment of all our document efforts, but that advocacy also needs to be more closely aligned with proactive team mentoring so members can collaboratively improve their practice. Members need to know the strategic direction of the company, actively negotiate their practice to align with that direction, and validate their evolving practice with customers. For team members to evolve their practice they need full knowledge of the direction of the laboratory and the areas targeted for future funding activities (Capezio, 1996).

\section{$\underline{\text { Initial Interviews }}$}

My fourteen initial informants are the cross-disciplinary, team members of the proposal service. The informants include seven editors, two graphic artists, two text processors, secretary, production coordinator, and team leader. Some interviewed members have been with the proposal team since it started three years ago; two have moved to other positions based on their capabilities in handling complex processes, and three had just started interacting with the team. Thus the data collected from the homogeneous informant pool provides both discipline and time perspectives on proposal practice.

The framing question, "What impact can cross-disciplinary team processes have on strategic organizational learning?" scaffolds the interview process. Interviews were conducted in a walled office for privacy and to promote candid dialogue. Test interviews involved three members of the core team, two editors and the secretary, to confirm the 
interview protocol evoked in-depth informant responses and covered proposal practice topics of importance to the informants. The initial questions used were

- What attributes are important for a member to possess to contribute to a proposal effort and why?

- What proposal service developed tools do you most frequently use and why?

- What features of the tools are most helpful? Which are least helpful?

- $\quad$ Based on your interactions with proposal service customers, what do they value most about the service? What do they find least beneficial?

- What personal value do you place on working with the proposal service?

Although these are the initial main questions, the interview process followed the ethnographic tradition. At the start of these three interviews, I invited each informant to provide feedback on the interview questions and suggest modifications and additions to the interview protocol. The initial informants indicated the questions adequately covered their contributions to the proposal service and provided them with opportunities to reflect on their practice. As a result of these interviews, I did add a final question inviting informant input on any topics or concerns not covered with the interview questions by asking

- Is there anything else you want to share with me about the proposal service that you have been thinking about and we haven't covered?

Although the question is not open-ended, I was comfortable posing it based on the homogeneous informants' level of candor during all interviews. Also, once the final interview questions were discussed, I turned off the tape recorder so informants would have an opportunity to share other concerns or comments in private. During this private sharing time, informants focused on their visions about the future of the proposal service, 
engaged in cordial personal conversation, and asked probing questions about upcoming proposal opportunities. I noticed no change in the informant's candor or engagement in the unrecorded interview process; thus, increasing my comfort with the honesty and candor of all data acquired during the taped portions of the interviews. The interview transcripts, transcript memos, and my personal journal provide the meta-inquiry data for both the topic-oriented ethnography and grounded theory analysis and interpretation.

\section{$\underline{\text { Topic-oriented Ethnography }}$}

My research uses a facet of the ethnographic tradition, topic-oriented ethnography, to focus on an aspect of organizational life, proposal preparation, existing in the work community (Spradley, 1980). Topic-oriented ethnography captures three primary elements of the proposal community-place, participants, and activities. To value informant interview data and tell the informants' stories, I first looked at the cultures of the participants by discipline-graphic artists, editors, and text processors. Separate topicoriented ethnography studies were not performed on the production coordinator, secretary, and the team leader, as their data are included in the editor, text processor, and scientist cultures, respectively. Following heterogeneous informant interviews, topicoriented ethnography was expanded to include the cultures of scientists, engineers, and managers.

All proposal team members work in a research and engineering laboratory where science and engineering knowledge is valued more highly than communications, English, technical writing, art, text processing, or document production knowledge (Bennett, Fadil, \& Greenwood, 1994; Boyer, 2000). Although the scientist and engineer culture is critical for the laboratory, Ford, Voyer, and Wilkinson (2000) suggests, "that organizations with a balance among cultures may be more likely to succeed than those 
dominated by a single culture" (p. 72). Additionally, undervalued team members come to the proposal service from a technical services organization where each is viewed as an interchangeable, individual performer doing their piece in an unknown, bigger document preparation process. Thus, proposal service core members came to the service as ordinary employees with good talents, academic underpinnings aligned with their discipline, and a latent ability to move beyond the status quo. One graphic artist shared that before working with the proposal service they had never been called upon to work nights and weekends or to work on critical company funding document. For many years the artist worked a normal workday on routine work assignments of average difficulty.

The artist's work routine typifies the company's work culture-perform at an average level. Exceptional achievement or living on an adrenaline high is not expected or, in many cases, rewarded, so average performance is the norm (Bennett, Fadil, \& Greenwood, 1994). From this company culture of "the average," the proposal team was assembled and asked to work interdependently to move beyond the status quo and excelthe artist contributing from the unknown viewpoint, the editor from the constructing viewpoint, and the text processor and document production staff from the presentation of the whole viewpoint (Brown, 1996).

\section{Graphic Artist}

The graphic artist is a "visual person" a who is generally introverted and a bit self-doubting. There is a tendency to convey, both verbally and nonverbally, that the job of illustrating a concept is initially overwhelming to them causing a customer to feel uneasy about the artist's ability to produce the needed illustration. As the artist takes the

\footnotetext{
${ }^{a}$ Quotation marks are used when presenting comments directly from transcribed informant interviews, researcher journal, or proposal team debriefing minutes.
} 
first steps in their creative process, they appear to hit a mental erase button and visualize on a blank screen. Self-doubt results from the fact that the artist is mentally creating an image. One graphic artist describes the concept visualization by stating,

"Processes are hard. Intellectual material, intellectual resources, those are hard. Logos, logos are a lot more difficult than they appear because there is usually a concept. There is not usually a physical product. It is people and it is processes, and to try to grab something out of that, that to me at least says everything that you want to say, is real difficult. So those things are tough.”

The artist places great value on dialogue and sketches to visualize a concept before committing to a path forward. The interplay between artists is important to their success as one states, “he has some talents that I don't have and he doesn't try to take over parts and I hope I don't take over from him." Fellow artists dialogue to visualize the concept as this graphic artist explains,

"If I bounce them [ideas] off of him [artist] or he with me, then I start seeing what I want to do with it. Where he may never lay down a single line, having had him there is something that has increased my confidence and I feel inclined to go ahead."

The artist values the editor's interpretative abilities to clearly explain a complex concept the author is trying to convey. One artist explains, "I usually work more directly with the editor, because I think the editor has partly distilled down what the guy [author] wants to say without me getting involved."

As with dialogue, "a rough sketch with stick figures or a few boxes" can also move the artist forward. A text description can be helpful if it is "a concise little synopsis, 
that is not a problem. It is when I have a stack of papers; that just overwhelms me." Once the artist establishes the approach to a complex illustration, the value of dialogue diminishes and the artist wants "to be just left alone...because it's a complicated piece and I am thinking my way through it."

Perfection is not the artist's goal in their first rendering; rather the rendering stimulates further dialogue. It is "something to talk about and know this isn't it, then okay, why isn't that it and you start to get something. I try not to polish it too much at that point."

As many graphics are rendered using computer packages, the graphic artists feel their contribution is small because "half the time the computer will do most of the work for you." Software packages also cause the artist to undervalue their gifts. Both interviewed artists expressed the thought that "I am not sure that talent has a whole lot to do with [it]...I don't really see that I have any particular gifts."

But not all artist contributions involve creating an original illustration of a concept; sometimes they need to enhance the look of a simple graphic, such as a flow diagram. Although there is little creativity in this process, the artist places pride in their ability to "make it look a little better than it might otherwise normally, but it is really not a creative process."

Illustrating concrete objects is a true delight for the graphic artist because, "I could visualize it. I could pull it together from blueprints and it was real as if we had actually built it." On one occasion, an artist team worked on "Virtual Ville... it was fun and we were building buildings and we were putting up signs and putting up railroad tracks and it was like building a little toy town." The same artist team worked closely with a laboratory engineering team to illustrate over $603-\mathrm{D}$ graphics in a conceptual 
design document. The graphics clarified and amplified the design text. An engineer working with the artists indicated, "it was easy to figure out what you were going to write next because you were just explaining the figures." The synergy and excitement in this creative process was obvious as the artists enthusiastically put in long hours collaborating closely with the engineers to render accurate illustrations aligned with engineering drawings. The result of the effort was spectacular. One illustration has been used both nationally and internationally in journal articles and press releases. Additionally, the manager on the engineering project indicated that the regulators clearly understood the engineering concepts because of the graphical presentation. That understanding accelerated approval of the concept and receipt of funds.

Graphic artists enjoy the freedom to think and create and make tacit ideas or concepts, the "unknown," explicit through their art. The artist is the right-brained thinker on the proposal team and complements the left-brained editor contribution. The artist synthesizes proposal graphics from an intuitive and holistic perspective while the editor rationally and analytically assembles each part of the proposal (Dwyer, 1998; Funderstanding, 2003).

$\underline{\text { Editor }}$

The editor functions in the role of liaison between various contributors (Mead, 1998). Dialoguing with the artist, the editor interprets graphics that illustrate and amplify the author's text. As the author dialogues about their concept, the editor mentally crafts the document and sales pitch using the proposal requirements as the scaffolding. The proposal editors must construct a document linking text and graphics, leading the reader through the proposal. Because the editor is constructing the document on a known scaffolding, confidence is conveyed to the author. One editor explains, "Proposals are a 
challenging change...I also get to work with different people, who are exceptionally smart, challenging, and interesting. This change makes me step out of my comfort zone and makes my job much more interesting."

Senior editors actively mentor junior editors. Mentor/intern pairs work synergistically on major document efforts allowing junior editors to quickly enhance their value to the team. One intern provides insight into this unique aspect of the editor culture.

"I think it was so smart when you paired us up. At least for me, being new. Being able to work with [the senior editor] has been really great for me because if I have even some minor question that I don't know what the company standard is, you know, I can go to [the senior editor] and he can generally just know right off the top of his head. He knows what it is and then he knows where he can find it to show me for sure. So that has been really helpful. I have really appreciated having him right there. It is really nice."

Proposal service editors are the exception to the image of an introverted, solitary wordsmith who is mildly anti-social, strongly opinionated, and inflexible and assumes they are the keeper of the keys to the English language (Henry, 1998; Hughes, 2002). The proposal service editors work together to improve their practice, and they are very customer oriented. This synergy results in improved practice because

"Working with other VPS [Virtual Proposal Service] editors helps me become a better editor... when supporting VPS, I get to interface with the best [editors], which in turn pushes me to also be the best. The other VPS editors have been very helpful. Typically, editors are not willing to help 
each other or share information. This has not been the case with VPS editors."

Because each proposal editor works closely with an author or authors to construct a proposal, the editor is sensitive to the fact that "proposal authors have a strong stake and interest in producing the best document possible because their career depends on it. This gives me incentive to be the best editor I can." Editors must work closely with the author to understand the scientific and engineering concepts, to tailor their assistance to complement the writing skills of the author, and to sell the author's concepts by crafting powerful text amplified by graphics. Proposal team editors must "enjoy very much learning about the different fields" and have the intellect to be comfortable working in variety of areas "totally out of reach of anything that [they] have worked with before."

The editor must have the ability to dialogue with the graphic artist about a visual concept rather than presenting a visual person with pages of text and, at the same time, craft compelling text for the author. Some authors are not skilled in leading the reader through their proposal concept so the editor focuses on "things that need to be reorganized so that the reader can follow the story. If that is all I have time to do is get a story out of it...I am just happy if I have a story at all.”

Besides functioning as the liaison with all contributors to a proposal, the editor must pay careful attention to all the agency solicitation requirements to produce conformal, responsive proposal text. Thus, there are "times the editors like to be more left alone to have their time to read it [the proposal] through themselves without having all the input."

Document coordination and text preparation fall squarely on proposal editors who are competent, cheerful, easy going, and customer oriented. They enjoy the fact that they 
are "working on documents that actually have an impact on the company. So, personally that makes me feel good." The team leader provides each editor with the opportunity to participate in all aspects of the document production process. Production is full of timeconstrained steps so editors need learn to how to balance their passion for crafting perfect text with the time constraints of production.

The editors work closely with the team leader to develop tools to save authors and editors time by speeding up and enhancing document preparation, technical review, and production. Editors prefer working on documents with proposal team generated and tailored guidance, template, and checklists. The core team editors "really like getting into the call and analyzing it and focusing it down to, now given all these overblown wordy calls, what do we really need to focus on here."

In addition to document tools, the editors identify ways to automate repetitive processes such as references and acronym lists, develop desk reference guides to clarify common questions, prepare electronic resources for authors, and enhance current proposal tools. Editors proactively work to improve their practice and work collectively to push the status quo. Because editors work in the world of text, they predominately use left-brain thinking skills to make the proposal process as explicit as possible. Thus, with the combination of the graphic artist and the editor culture, the proposal service team "thinks" using the entire brain to produce a quality, well-crafted document that resonates with both the textual or graphical comprehension of reviewer.

\section{$\underline{\text { Text Processor }}$}

The text processor integrates the text and graphics into the "presentation of the whole." Besides the responsibility for document layout, the text processor is faced with having to perform under extreme time constraints. On large proposal efforts, the text 
processors must integrate hundreds of pages of text and dozens of graphics using company standard software that is ill suited for complex document layout. In spite of these incredible time and technical challenges, proposal team text processors are easygoing, friendly, and a calming force in crisis. One text processor explains, "I really enjoy the text processing part of it because I actually feel like I am doing something for the document."

On the team, the text processors hold a revered spot by all team members. Because of their demeanor and poise in crisis, all team members honor the processors need to have adequate time to produce a quality layout. Team members will work weekends and late into the night to provide the text processors with completed text and graphics.

The processor's job requires either solitude or dialogue. One text processor describes,

"If it is just the little, you know, change a space here or formatting, it is better to just be left alone, because if you get interrupted then it is ' $\mathrm{Did}$ I do this part? How far did I get?' But if it is rewording and making sure that you have it correctly worded and you are getting the right meaning across, it is better to have you sitting with me."

Even with the editors and artists working to have text and graphics complete, the text processors work long hours and are generally the last team member working on the document before it goes to the print shop. One text processor mentioned the fact that team members are hesitant to leave while the processor is still working on a document. The hesitation springs from proposal team member's commitment to the document, their 
willingness to assist in anyway possible to produce a quality document, and their sense of caring about other team members (Liedtka, 1999).

\section{Topic-oriented Ethnography Summary of Homogeneous Informant Data}

The graphic artist, editor, and text processor cultures blend as team members appreciate each other's contributions and enjoy working together on demanding documents. They are proud of their contribution to the future of the company because they assist in securing new research and engineering funds. This pride is not based on being mentioned by name in press releases, but rather personal pride in knowing they helped when proposal wins are announced.

Looking at the culture of the proposal team members reveals that informants view themselves as participants in the process of crafting a holistic artifact. The proposal service weaves a proposal fabric prescribed by proposal guidelines and requirements with the author and team members collaborating to add unique texture and design to that fabric. The result is an aesthetic whole that integrates concept, structure, form, and unity (Lawrence-Lightfoot \& Davis, 1997). Figure 6 illustrates the creation of an integrated whole, the proposal document.

The author provides the concept, the basis for the proposal process, with their innovative and compelling research idea or engineering approach. The editor provides a structure for the author's concept. Part of the structure is dictated by the funding agency's proposal requirements, but the editor, in collaboration with the author, crafts a 


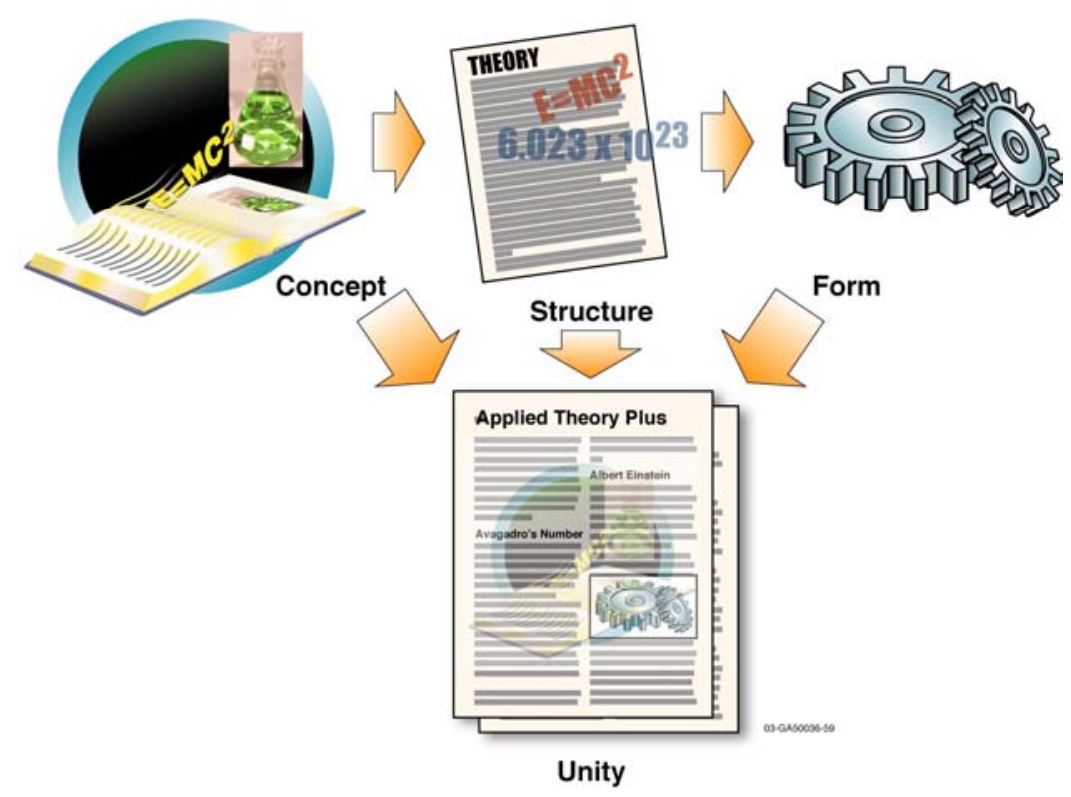

Figure 6 . The author provides a concept; the editor provides structure; the artist provides form; the text processor provides unity.

unique proposal fabric to clearly and concisely portray the author's concept. One editor explains their collaboration with an extremely bright author.

“The technical ideas were there; he [author] just couldn't get them from his head on to paper so that it made sense. So he appreciated being able to sit down and talk with me and have me go off and write it and then give it back to him and say, 'Is this what you said?' Because he couldn't transfer his thoughts into words on paper."

The graphic artist provides a pleasing, artistic form to complete and amplify the textural structure. Although the text tells a story in sentences, a well-drawn graphic brings those words to life. One of the graphic artists puts it this way 
"That was so much fun because I could see what it was we were doing. I could visualize it. I could pull it together from blueprints and it was real as if we had actually built it. But it was also the fact that, I mean, we are talking about a $75 \%$ reduction in wastewaters that contaminate the world and I thought that was worthwhile. I am thinking we can sell this to guys if we could just tell it to them [graphically] the way it is."

A proposal is the product of a cross-disciplinary team including the author, editor, graphics artist, text processing, technical reviewer, technical manager, and the proposal service technical leader (Mead, 1998). Through the layout process text processing integrates the text and graphics into the final fabric with its unique texture and design giving unity to the proposal. One editor explains the value of layout assistance by stating, "I would say the mechanical assistance they [authors] appreciate most." The artistic form provided by graphics and layout adds branding to the document and provides visual intrigue to compel a reviewer to read the document.

The final production step, printing, completes the concept, structure, form, and unity by revealing the clarity, responsiveness, and power of the text; portraying the richness of the graphics; and presenting a quality hardcopy volume or electronic submittal file for critical evaluation by reviewers and funding by the soliciting agency.

From the interviews with proposal team members, each member is extremely proud of their contributions to our final product, a compelling document requesting funds for the laboratory! A team member explains, "They [authors] have a product that they are proud of when it is out the door. Quite often they also have very appreciative customers, which is nice." 
One source of that pride is the creation of a coherent, holistic suite of documents that communicate the proposal authors' scientific logic and creativity. All documents are conformal and responsive because authors can think and rethink, write and rewrite, then, read and reread, as does the editor who also coordinates the document process. One informant stated that

"The most valuable is the editor, in the sense of the editor taking all the burden off of their [authors] shoulders of the coordination, of lining up the text processor, writing even, other than their technical part, which is their area of most interest and their area of expertise. And so they [authors] of course reviewed it and passed it on, but didn't have to worry about it and sit down and mess around with the bios, for example, and the introductory stuff and the sales pitch.”

Reviews also ensure documents are coherent. Technical reviews conducted by company senior scientists and engineers provide new perspectives on the concept, structure, and form to enhance the presentation. The review process is an anxious time for the author and editor team as one member explains,

"The value on the reviews, if it works, if it gives them [author and editor] a buffer and they get good reviews without hurting their egos, or something. That is probably one of the things that does them the most good and yet they resist the most, is to use a systematized technical review process."

Another aspect of coherence results from the team leader looking across all critical documents to ensure there is consistent textural, graphical, and format quality; 
thus, providing a uniform company brand to promote recognition of company documents by funding agencies. My journal entry captures this thought.

"The product [all critical funding documents] is the future of our effort-not only the wins. The wins will come as we portray the quality of our research and engineering ideas. I believe the same quality ideas existed before our proposal service, but now proposal reviewers 'look forward' to reviewing our proposals because they are well written, conformal, responsive, and visually appealing."

\section{Meta-inquiry}

Meta-inquiry coupled with topic-oriented ethnography increases the researcher's theoretical sensitivity to the human ecology by initial efforts to frame context, dialogue with informants, evaluate the questioning process, and explore related topics in the extant literature (Glaser, 1978). Enhanced researcher sensitivity is essential for the emergence of a theoretical position of true significance to the researched human ecology following meta-inquiry (Carlson \& McCaslin, in press).

Meta-inquiry uses the analysis and interpretation approaches of the grounded theory tradition. Analysis blends open and reflective coding, moving the research between deductive and inductive thought. The product of the open and reflective coding portions of meta-inquiry forms an analytical and interpretive complex constructed from initial interview protocol data. The complex becomes critical for moving a grounded theory study beyond the creation of a theoretical position towards a workable, generalizable, and testable theory truly grounded within the human ecology (McCaslin, 1996). Meta-inquiry adds an essential step to grounded theory by reconciling initial interview data acquired from a homogenous group of informants and deepening 
our understanding of the human ecology through constant comparison (Glaser \& Strauss, 1967). This human ecology-based awareness is best achieved by paying attention to the culture, habits, and traditions of the environment in which we find ourselves (McCaslin, 2002). Additionally, meta-inquiry provides the reflective pause necessary to see the connections between initial informant data and the environment. The result of meta-inquiry is a theoretical position. The theoretical position coupled with theoretical conditioning and selective questioning, results in the development of an ecology-based grand tour question that frames the phenomenon of interest and elicits rich data from a heterogeneous informant pool (Carlson \& McCaslin, in press).

Meta-inquiry compared and connected the homogeneous informant interviews, researcher interview memos, and researcher journal entries using an open and reflective coding process linked by constant comparison. The deductive, open coding process is "the analytic process through which concepts are identified and their properties and dimensions are discovered in data" (Strauss \& Corbin, 1990, p. 101). I chose to do lineby-line analysis to identify all concepts valued by the informants. Glaser (2001) relates that

The true and only source of the core category is the rigorous use of the GT [grounded theory] method starting with open coding using line by line constant comparisons. False core categories can lead to a confusion between open and selective coding. (pp. 200-201)

As part of the open coding analysis, I added researcher memos to interview data as process concepts emerged, added researcher insight on a recounted incident, or simply reflected on the interview. Glaser \& Strauss (1967) share the value of memoing when they explain, 
The second rule of the constant comparative method is: stop coding and record a memo on your ideas. This rule is designed to tap the initial freshness of the analyst's theoretical notions and to relieve the conflict in his thoughts. (p. 107)

Reflective coding, defined as "the process of putting the data back together in meaningful ways by making connections between a category, its dimensions, and the context in which it is found" (McCaslin, 1995, p. 63), reassembles the data fractured during open coding. Open and reflective coding occur concurrently as the data are fractured and reassembled, respectively. Constant comparisons help clarify concepts and reveal relationships between concepts resulting in the emergence of categories, properties, processes, and dimensions. As reflective coding reveals these relationships they are logically organized using reflective coding steps shown schematically in Figure 7.

Discovering process concepts was the focus of open coding as the category scaffolding-proposal team core group values, and the properties-proposal team attributes and tools, quickly emerged from the initial three interviews. The scaffolding category and the properties remained constant throughout meta-inquiry open and reflective coding of the remaining homogeneous informant interviews. 

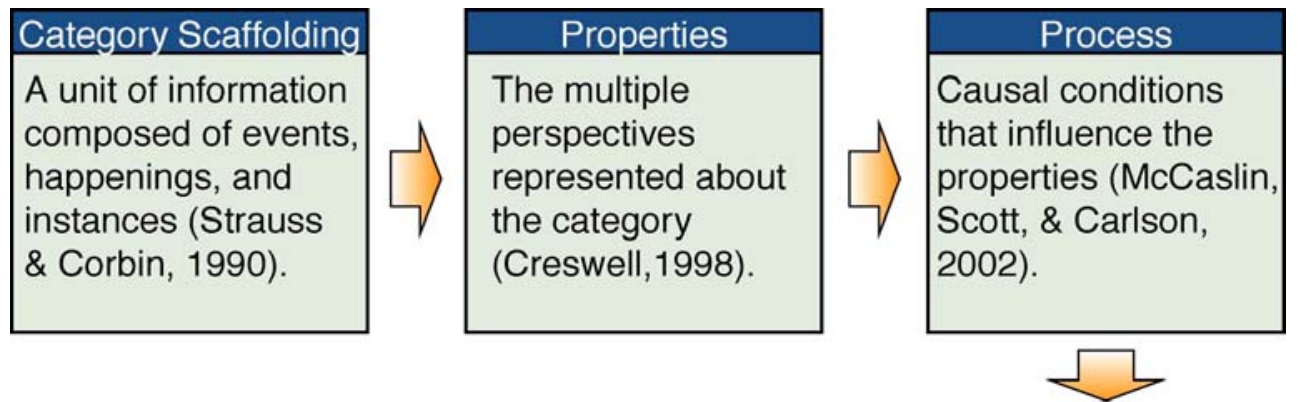

Consequences

A range of conditions influencing and delineating the outcomes of strategies (Creswell, 1998).

\section{Context}

The setting for data analysis and interpretation (Boyatzis, 1998).
Dimensions

The range of all possible aspects of a process (Creswell, 1998).

Figure 7. Reflective coding matrix logic flow.

I used QSR NUD*IST Vivo, a qualitative software package, during open coding to allow for maximum flexibility in establishing, renaming, separating, and merging process concepts during reflective coding (Fraser, 1999). Identifying informant-valued, in vivo phrases assisted me in establishing the names of seven process concepts

- $\quad$ Service oriented

- $\quad$ Contribute to the future of the laboratory

- $\quad$ Dedicated team member

- $\quad$ Exciting challenge

- $\quad$ Speed up process

- $\quad$ Barriers

- $\quad$ Experience

Before proceeding further with the inductive process of reflective coding, I needed to establish the robustness of these seven process concepts. Developing a conditional relationship coding matrix clarified the robustness of an initial process 
concept as I focused on the what, when, where, why, and how questions related to the initial concepts, as well as the consequence of each concept (Scott, 2002). Using the conditional relationship matrix allowed me to determine if an identified process is truly a process, i.e. all the relationship questions can be answered uniquely, or if the initial process is rather a dimension of an overarching, yet to be discovered, process. The matrix also provides an opportunity to determine the richness of a process and its dimensions. If the dimensions of a process are few, making the process thin, additional interview data are considered necessary to add richness to the process. Table 2 provides the conditional relationship matrix for the initial seven process concepts identified using the category scaffolding of the proposal team core group values. 
Table 2

Conditional relationship coding matrix

\begin{tabular}{|c|c|c|c|c|c|c|}
\hline Category & What & When & Where & Why & How & Consequence \\
\hline Service Oriented & $\begin{array}{l}\text { Gain buy-in from } \\
\text { customers, } \\
\text { dialogue with } \\
\text { customers, be } \\
\text { flexible, cheerful, } \\
\text { easy going, } \\
\text { professional } \\
\text { demeanor, do } \\
\text { whatever is } \\
\text { needed, have } \\
\text { depth of coverage } \\
\text { so no work flow } \\
\text { stops }\end{array}$ & $\begin{array}{l}\text { From the first } \\
\text { meeting with } \\
\text { authors/managers } \\
\text { through project } \\
\text { completion, in } \\
\text { VPS team } \\
\text { meetings both } \\
\text { general topic and } \\
\text { document specific }\end{array}$ & $\begin{array}{l}\text { One senior } \\
\text { manager } \\
\text { identified and } \\
\text { sanctioned } \\
\text { documents }\end{array}$ & $\begin{array}{l}\text { Reduce stress on } \\
\text { author so they } \\
\text { can concentrate } \\
\text { of the technical } \\
\text { content, increase } \\
\text { quality of } \\
\text { product, value } \\
\text { author's unique } \\
\text { ideas, to build a } \\
\text { teaming between } \\
\text { VPS and authors, } \\
\text { need all on team } \\
\text { to put in the time } \\
\text { needed to product } \\
\text { a quality product }\end{array}$ & $\begin{array}{l}\text { Asking probing } \\
\text { questions of author, } \\
\text { gain trust of } \\
\text { customers by } \\
\text { producing quality } \\
\text { document, being } \\
\text { responsive and } \\
\text { listening to their } \\
\text { concerns }\end{array}$ & $\begin{array}{l}\text { Customer } \\
\text { confirmation of } \\
\text { quality product }\end{array}$ \\
\hline $\begin{array}{l}\text { Contribute to the } \\
\text { Future of the } \\
\text { Laboratory }\end{array}$ & $\begin{array}{l}\text { Assist in obtaining } \\
\text { future funds by } \\
\text { using our service, } \\
\text { working critical } \\
\text { documents, we are } \\
\text { on the "inside" of } \\
\text { the future of the } \\
\text { lab, raising quality } \\
\text { of critical funding } \\
\text { document }\end{array}$ & $\begin{array}{l}\text { All our documents } \\
\text { are adding value } \\
\text { as someone reads } \\
\text { them and makes } \\
\text { funding decisions } \\
\text { that impact the } \\
\text { company's future }\end{array}$ & $\begin{array}{l}\text { With all agencies } \\
\text { that use } \\
\text { documents to } \\
\text { make laboratory } \\
\text { funding } \\
\text { decisions }\end{array}$ & $\begin{array}{l}\text { To secure lab } \\
\text { funds, to have } \\
\text { our documents } \\
\text { read, understood, } \\
\text { and reviewed } \\
\text { favorably, to } \\
\text { establish a } \\
\text { laboratory quality } \\
\text { level for critical } \\
\text { documents, to } \\
\text { secure our jobs, } \\
\text { to have high-end }\end{array}$ & $\begin{array}{l}\text { We bring funds to } \\
\text { the laboratory! } \\
\text { agency } \\
\text { acknowledgement of } \\
\text { the quality } \\
\text { documents we } \\
\text { produce, agency } \\
\text { acknowledgement of } \\
\text { the clarity of the text } \\
\text { and presentation, } \\
\text { customers verbalize } \\
\text { their appreciation for }\end{array}$ & $\begin{array}{l}\text { Confirmation of } \\
\text { increased } \\
\text { document } \\
\text { quality and } \\
\text { effectiveness }\end{array}$ \\
\hline
\end{tabular}




\begin{tabular}{|c|l|l|l|l|l|l|}
\hline Category & What & When & Where & Why & How & Consequence \\
\hline & & & & work & the assistance & \\
\hline
\end{tabular}

Table 2

Conditional relationship coding matrix (continued)

\begin{tabular}{|c|c|c|c|c|c|c|}
\hline Category & What & When & Where & Why & How & Consequence \\
\hline $\begin{array}{l}\text { Dedicated Team } \\
\text { Members }\end{array}$ & $\begin{array}{l}\text { Part of a strategic } \\
\text { group, camaraderie, } \\
\text { members with } \\
\text { diverse skills }\end{array}$ & $\begin{array}{l}\text { Working on } \\
\text { critical } \\
\text { documents, } \\
\text { solving process } \\
\text { issues together }\end{array}$ & VPS team & $\begin{array}{l}\text { "I" am } \\
\text { contributing to } \\
\text { the team's } \\
\text { success, all } \\
\text { members' skills } \\
\text { blend to create a } \\
\text { quality whole, } \\
\text { willing to do the } \\
\text { extras needed to } \\
\text { get the job done }\end{array}$ & $\begin{array}{l}\text { Doing my part well, } \\
\text { helping others when } \\
\text { needed, using others } \\
\text { as a sounding or } \\
\text { spring board to do my } \\
\text { part better }\end{array}$ & $\begin{array}{l}\text { Ownership and } \\
\text { pride in } \\
\text { resulting } \\
\text { document }\end{array}$ \\
\hline $\begin{array}{l}\text { Exciting } \\
\text { Challenge }\end{array}$ & $\begin{array}{l}\text { Stretches the team } \\
\text { members' talents, } \\
\text { focuses their } \\
\text { efforts, challenges } \\
\text { their intellect }\end{array}$ & $\begin{array}{l}\text { Take the abstract } \\
\text { and make it } \\
\text { concrete with } \\
\text { graphic/text, } \\
\text { learning about } \\
\text { new science and } \\
\text { engineering } \\
\text { technologies, } \\
\text { work needed } \\
\text { hours }\end{array}$ & $\begin{array}{l}\text { Throughout the } \\
\text { document process }\end{array}$ & $\begin{array}{l}\text { Like the } \\
\text { excitement, use } \\
\text { creative license, } \\
\text { creates something } \\
\text { different, given } \\
\text { responsibility, } \\
\text { job satisfaction, } \\
\text { enthusiasm, have } \\
\text { fun }\end{array}$ & $\begin{array}{l}\text { Get recognition for } \\
\text { customers, } \\
\text { recognition better } \\
\text { than money, have a } \\
\text { stake in the } \\
\text { document's success, } \\
\text { gives incentive to do } \\
\text { a good job, working } \\
\text { on something that } \\
\text { matters to the } \\
\text { laboratory }\end{array}$ & $\begin{array}{l}\text { Enhanced job } \\
\text { satisfaction }\end{array}$ \\
\hline
\end{tabular}




\begin{tabular}{|l|l|l|l|l|l|l|}
\hline Category & What & When & Where & Why & How & \\
\hline & & & & & & \\
\hline
\end{tabular}


Table 2

Conditional relationship coding matrix (continued)

\begin{tabular}{|c|c|c|c|c|c|c|}
\hline Category & What & When & Where & Why & How & Consequence \\
\hline $\begin{array}{l}\text { Speed up } \\
\text { Process }\end{array}$ & $\begin{array}{l}\text { Provide tools as } \\
\text { well as a process } \\
\text { with experts in all } \\
\text { steps, give authors } \\
\text { a writing strategy, } \\
\text { can provide } \\
\text { examples, } \\
\text { proactively monitor } \\
\text { document process } \\
\text { to avoid crisis, } \\
\text { identify needed } \\
\text { tools and develop } \\
\text { those needed with } \\
\text { senior management } \\
\text { guidance }\end{array}$ & $\begin{array}{l}\text { From the kick- } \\
\text { off meeting } \\
\text { through } \\
\text { production, as } \\
\text { document } \\
\text { evolves we } \\
\text { know the } \\
\text { content so we } \\
\text { can restructure } \\
\text { when needed to } \\
\text { provide a better } \\
\text { story }\end{array}$ & $\begin{array}{l}\text { Co-location } \\
\text { of the VPS } \\
\text { team in } \\
\text { October } 2002\end{array}$ & $\begin{array}{l}\text { Organize the proposal } \\
\text { or document to meet } \\
\text { the agency } \\
\text { expectations, achieve } \\
\text { required timeline, } \\
\text { give all members } \\
\text { time to do their job, } \\
\text { "control" the process, } \\
\text { clarify team } \\
\text { members' roles, get } \\
\text { responsible manager } \\
\text { as an active partner to } \\
\text { guarantee success }\end{array}$ & $\begin{array}{l}\text { Take the burden off the } \\
\text { authors by preparing } \\
\text { the template, guidance, } \\
\text { checklist, timeline, } \\
\text { team list, all tools on a } \\
\text { public shared server, } \\
\text { help authors to clarify } \\
\text { thoughts, provide the } \\
\text { "mechanical" } \\
\text { assistance, coordinate } \\
\text { the process }\end{array}$ & $\begin{array}{l}\text { Quality product } \\
\text { handled by } \\
\text { experts }\end{array}$ \\
\hline
\end{tabular}




\begin{tabular}{|l|l|l|l|l|l|l|}
\hline Category & What & When & Where & Why & How & Consequence \\
\hline & & & & & & \\
\hline
\end{tabular}


Table 2

Conditional relationship coding matrix (continued)

\begin{tabular}{|c|c|c|c|c|c|c|}
\hline Category & What & When & Where & Why & How & Consequence \\
\hline Barriers & $\begin{array}{l}\text { Impede the } \\
\text { production of a } \\
\text { quality document, } \\
\text { some customers } \\
\text { have a } \\
\text { misconception of } \\
\text { the VPS process } \\
\text { and level of } \\
\text { customer service, } \\
\text { stress on team } \\
\text { members, some } \\
\text { work is } \\
\text { "production" } \\
\text { level and does } \\
\text { not require } \\
\text { creativity, some } \\
\text { authors do not } \\
\text { see their blind } \\
\text { spots so it's hard } \\
\text { to work assist } \\
\text { them, current } \\
\text { production } \\
\text { software is awful, } \\
\text { time to } \\
\text { concentrate on } \\
\text { your job }\end{array}$ & $\begin{array}{l}\text { At the production } \\
\text { phase of the } \\
\text { process } \\
\text { especially, if } \\
\text { authors are } \\
\text { "forced" by } \\
\text { managers to use } \\
\text { VPS it is hard on } \\
\text { the editors, } \\
\text { customer can't } \\
\text { articulate concept } \\
\text { so must pull } \\
\text { information from } \\
\text { them, adverse } \\
\text { feelings about } \\
\text { technical reviews } \\
\text { (author egos are } \\
\text { fragile) and } \\
\text { literature } \\
\text { searches, authors } \\
\text { who do not know } \\
\text { how to write a } \\
\text { persuasive } \\
\text { document }\end{array}$ & $\begin{array}{l}\text { Throughout the } \\
\text { process }\end{array}$ & $\begin{array}{l}\text { Time constrained } \\
\text { process in a } \\
\text { laboratory culture } \\
\text { that is not used to } \\
\text { meeting firm } \\
\text { deadlines, need more } \\
\text { backup resources, } \\
\text { Word is not } \\
\text { production friendly- } \\
\text { wastes our time, extra } \\
\text { hours needed beyond } \\
\text { the normal work day } \\
\text { can be draining, VPS } \\
\text { team leader lacks the } \\
\text { "power" to direct the } \\
\text { process as authors } \\
\text { can whine to } \\
\text { managers and have } \\
\text { internal deadlines } \\
\text { moved! }\end{array}$ & $\begin{array}{l}\text { Editors need to } \\
\text { customize author } \\
\text { interactions and } \\
\text { assistance based on } \\
\text { the writing skills of } \\
\text { the authors, managers } \\
\text { need to work } \\
\text { collaboratively with } \\
\text { the VPS team leader } \\
\text { to achieve a common } \\
\text { goal }\end{array}$ & $\begin{array}{l}\text { Currently work } \\
\text { "too" hard to } \\
\text { produce a quality } \\
\text { product }\end{array}$ \\
\hline
\end{tabular}


Table 2

Conditional relationship coding matrix (continued)

\begin{tabular}{|c|c|c|c|c|c|c|}
\hline Category & What & When & Where & Why & How & Consequence \\
\hline Experience & $\begin{array}{l}\text { Performers must } \\
\text { be expert in their } \\
\text { unique area, } \\
\text { competent, be } \\
\text { viewed by } \\
\text { customers as } \\
\text { capable, must } \\
\text { have appropriate } \\
\text { education, be } \\
\text { bright, have } \\
\text { experience }\end{array}$ & $\begin{array}{l}\text { Must be able and } \\
\text { have the } \\
\text { knowledge to } \\
\text { complete their } \\
\text { part, work } \\
\text { effectively with } \\
\text { customers }\end{array}$ & $\begin{array}{l}\text { Throughout the } \\
\text { process }\end{array}$ & $\begin{array}{l}\text { Free technical } \\
\text { contributors to focus } \\
\text { on their part of the } \\
\text { knowledge } \\
\text { generation process, } \\
\text { free author from the } \\
\text { mechanics, get } \\
\text { experienced people to } \\
\text { do a good job on their } \\
\text { portion }\end{array}$ & $\begin{array}{l}\text { Work with the } \\
\text { customer to develop } \\
\text { graphics and text to } \\
\text { support and sell their } \\
\text { idea, allow all on team } \\
\text { the freedom to think } \\
\text { and create, each expert } \\
\text { focuses their efforts to } \\
\text { improve their part and } \\
\text { allows all the freedom } \\
\text { to concentrate their } \\
\text { talents }\end{array}$ & $\begin{array}{l}\text { Cross- } \\
\text { disciplinary } \\
\text { experts blend } \\
\text { talents to create } \\
\text { the whole }\end{array}$ \\
\hline
\end{tabular}


Community of Practice 64 
Grouping the consequences of the conditional relationship matrix revealed the casual condition themes of customer orientation, pride in practice, and focus on process enhancements recurred. By regrouping the original seven processes around these causal conditions, the original seven concepts collapsed into three processes with unique dimensions shown in Figure 8. All dimensions fit under one of these three processes. Service oriented and contribute to the future of the laboratory combined to form the process of customer focus and confirmation; dedicated team members, exciting challenge, and experience combined to form the process of whole created by experts; speed up process and barriers combined to form quality proposal product.

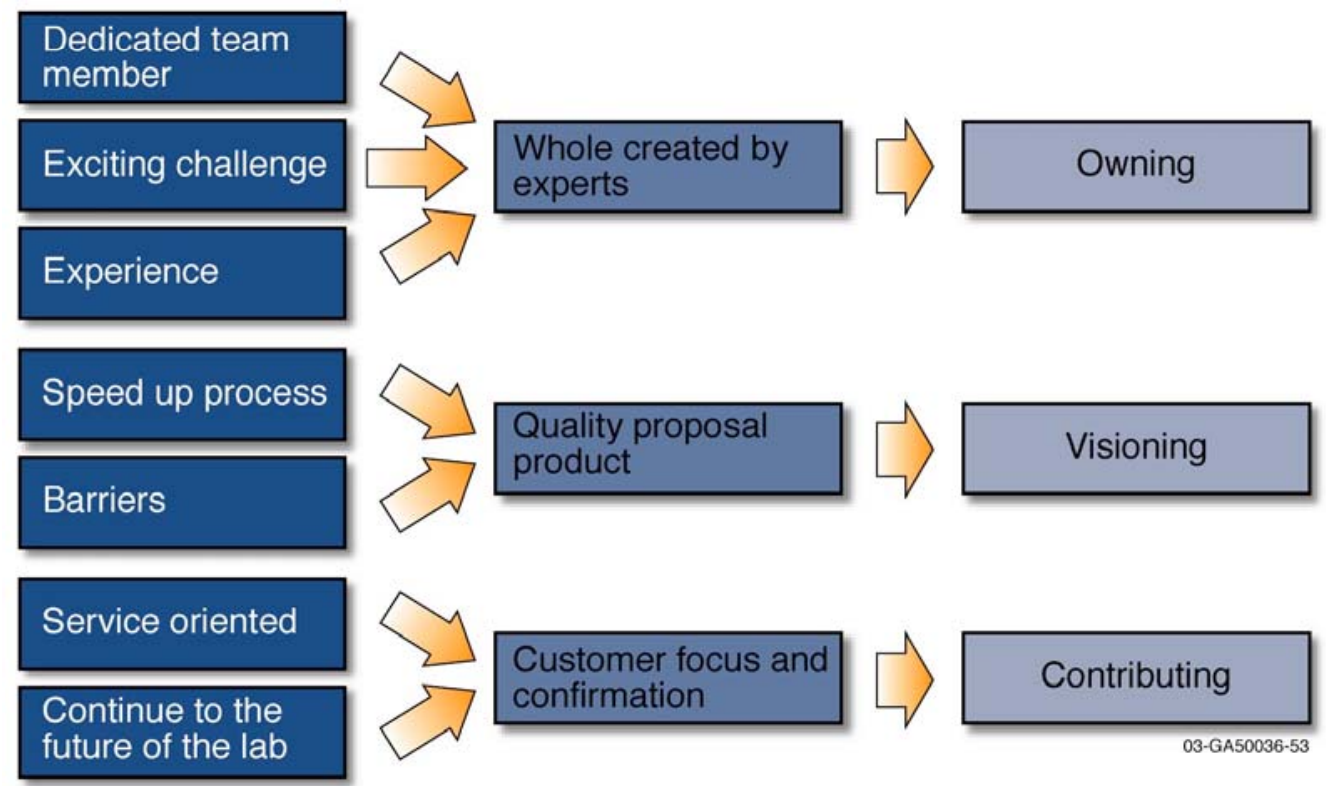

Figure 8 . Emergence of meta-inquiry process concepts using the conditional relationship and reflective coding analysis.

NVivo allowed processes to be easily merged and renamed. And merging provided another opportunity to reflect on informant data, recode some data to better 
align with the new process headings, establish the richness of all process dimensions, and confirm the fit of all informant, memo, and journal data. Glaser (1992) states, In grounded theory, since all coding, analyzing and constant comparing is emergent, everything fits somewhere as categories and their properties are discovered. Fit is automatic or a concept would not emerge. Grounded theory is very economical on strain and time. (p. 88) As part of the fitting process, process titles of whole created by experts, quality proposal product, and customer focus and confirmation resulting from merging the initial seven concepts did not fully represent dimensions of each process. The dimensions reflected actions, enthusiasm, and passion rather than lifeless words and phrases. Because the dimensions describe the three actionable processes, the gerunds-owning, visioning, and contributing-better represented the processes. The emergence of the gerund process concept names resulting from the conditional relationship coding matrix and the metainquiry reflective coding matrix are shown in Figure 8.

The use of gerunds to describe the three process concepts-owning, visioning, and contributing-captures the personal, evolutionary, and dynamic nature of the proposal process as explained by the team members. These gerunds imply action and reflect the voice of the informants. The dimensions of each process, shown in the meta-inquiry reflective coding matrix in Table 3, provide additional insight into the process.

Owning

The dimensions of owning

- Working on a Team

- $\quad$ Personally satisfying

- $\quad$ Challenging learning 
- $\quad$ Being flexible 
Table 3

Reflective coding matrix for homogeneous informant data

\begin{tabular}{|c|c|c|c|}
\hline Category Scaffolding & \multicolumn{3}{|c|}{ Proposal Team Core Group Values } \\
\hline Properties & \multicolumn{3}{|c|}{ Proposal Team Attributes and Tools } \\
\hline Dimensions & $\begin{array}{l}\text { Working on a Team } \\
\text { Personally satisfying } \\
\text { Challenging learning } \\
\text { Being Flexible } \\
\text { Exciting } \\
\text { Capably doing the job } \\
\text { Willing to do extra } \\
\text { Having Fun }\end{array}$ & $\begin{array}{l}\text { Actively controlling the process } \\
\text { Selling the idea } \\
\text { Creating process tools } \\
\text { Valuing process tools } \\
\text { Allowing author to focus } \\
\text { Working with management } \\
\text { Enhancing practice }\end{array}$ & $\begin{array}{l}\text { Focusing on customer } \\
\text { Impacting the company's future } \\
\text { Validating customer feelings }\end{array}$ \\
\hline Context & \multicolumn{3}{|c|}{$\begin{array}{c}\text { Proposal/Critical Funding Document Process Described by Core Members of the Research and Applied } \\
\text { Engineering Laboratory's Proposal Service Community of Practice }\end{array}$} \\
\hline $\begin{array}{l}\text { Strategies for } \\
\text { Understanding the } \\
\text { Consequences }\end{array}$ & \multicolumn{3}{|c|}{ Success-Linked Team Dynamics for Proposal Team Community of } \\
\hline
\end{tabular}


Community of Practice 69 
- $\quad$ Exciting

- $\quad$ Capably doing the job

- Willing to do extra

- $\quad$ Having fun

capture the personal value team members place on their owning practice (McDonough, 2000). The team is dedicated to their practice which is demanding, challenging, time intensive, but fun. All members verbalized that the proposal process provides them with self-satisfaction because they use their high-end skills on documents.

They like "working in a team" because there is "a lot of really healthy camaraderie, support." To promote the value placed on working as a team, proposal editors, graphic artists, and one text processor were co-located and mentor/intern relationships established between senior and junior editors after the first three interviews. The mentor/intern relationships are designed to speed enculturation of the junior editors into the proposal practice (Carlson, May, Loertscher, \& Cobia, 2003). A junior editor explains the personal benefits realized. "I think it was so smart when you paired us up. At least for me, being new...we can learn together....It is really nice. It is easy to work with him and I don't feel like I can't put my two bits in."

Team members have "more visibility and people obviously see you in a different light if you work on that level of document. They just assume that you are capable...I like the responsibility of working a proposal." An artist describes "[I] feel like I am a part of it and that what I contributed is important. I enjoy that much more than monetary whatever." And the challenge of the proposal practice brings out the talents of the team as they like "the complex jobs." Also, they know the "proposal authors have a strong stake and interest in producing the best document possible because their [authors] career 
depends on it. This gives me incentive to be the best editor I can." Part of owning is working with customers who may not fully value team talents even though they each bring "a specific expertise to the team." Team members come to the team with "a really bright mind, a really good education and experience, or some real natural bent for what they do." They must "be flexible to some degree and willing to listen to others." Members "are not doing something that anybody could just step in and do."

Their practice requires a "willingness to work nights and weekends" as well as the "ability to hit the ground running, do whatever is needed, and not stop until it's done." Also there is "a lot of crunch time, so you have to be able to work with that." Even with the demands of the practice, "it is fun. Even though it is hard, and even though it is demanding, I think it is fun. That is a rarity."

\section{$\underline{\text { Visioning }}$}

Team members not only own their practice but they actively engage in visioning about that practice. The dimensions of visioning voiced by the informants are

- $\quad$ Selling the idea

- Actively controlling the process

- $\quad$ Creating process tools

- Valuing process tools

- $\quad$ Allowing author to focus

- $\quad$ Working with management

- $\quad$ Enhancing practice.

The tools developed by the team arise from the visioning process. The team works only on documents valued and sanctioned by senior management so the team engages in visioning to "add value to a business enterprise" (Mead, 1998, p. 353), the laboratory. 
The defined proposal process "is straightforward and fairly simple, so people get a good handle on where they are going." The basic tools of template, guidance, checklist, and timeline have been part of the practice since the proposal service started, but the evolution of these tools is the result of team members collaborating and listening to customer input (Hughes, 2002). All tools are developed by "begin [ning] with the end in mind" to save the customer and team time and to guarantee a conformal, responsive document (Covey, 1989; Mead, 1998; Redish, 1995). Team members responsible for developing the tools enjoy "getting into the call and analyzing it and focusing it down."

The focus of their visioning "generate[s] synergy to create a high-quality product." This synergy evolves by clearly defining the roles of all contributors to a proposal document, by "actively control [ling] the process as an accepted part of the team," by analyzing and preparing needed tools "from day one," by providing “just in time training" to head "them [customers] off a lot from doing the wrong thing," by "taking all the burden off of their [authors'] shoulders of the coordination" of resources, and by allowing the author to focus on the "technical part, which is their area of most interest and their area of expertise."

The job of selling the idea is taken very seriously. Text must "capture requirements, audience, [and] purpose up front," "look consistent throughout," be "easy to follow," and provide "clarity of the visual presentation linked with the text."

\section{Contributing}

The final process, contributing, speaks of the value of the practice. Redish (1995) indicates that contributing must be measurable-save time, increase end-user trust, reduce redo rate, or increase revenue-to add value to an enterprise. Service to the customer and assisting in bringing funds to the laboratory validate the team's efforts. Each team 
member verbalized a similar sentiment to this editor. "I like the satisfaction of having pleased a customer." Research by Carter-Scott (2000) discovered that there is a direct "relationship between employee satisfaction and customer satisfaction. Believe it or not, they are parallel. My research indicates that the way managers treat their employees is directly reflected in the way those same employees treat their customers" (pp. 87-88). Although Carter-Scott's research looks at a manager's impact on customer satisfaction, interviews with heterogeneous informants, customers, revealed they value the enthusiasm, capabilities, and dedication of the team.

All members "have to be personable because we are a service organization" and "able to talk with and get along with a variety of personalities... within our group and within the different customer groups." Non-core service providers that do not have these customer or team focus attributes are not involved in future proposal efforts as the "customer is first and foremost."

The team understands and embraces the importance of their practice as "the future of this company is riding on this (proposal service)." The team is "doing something that is contributing to the lab, not just showing up every day." A benefit of working on critical documents involving the future funds for the laboratory is "I really feel like I am on the inside, knowing what is going on." We receive confirmation from customers that our assistance is making a difference through emails, phone calls, hugs, awards, and inclusion in customer celebration parties, but more importantly, through a significant, measurable increase in proposal wins. Also, some funding agencies have told our senior managers "that the INEEL proposal quality is much improved and the increased quality is being recognized by funding agencies and in the recent awards. He [senior manager] has received specific comments from agency folks on the document quality." 


\section{Process Concepts Summary}

The proposal team is actively involved in owning, visioning, and contributing as part of their practice. Figure 9 relates the three processes. All members came to the team as ordinary employees, but collectively they have developed absorptive capacity and capability to recognize and value new knowledge. The team has moved beyond average and excels in their practice. Also, the proposal team has moved beyond a collection of competent individuals working independently to an integrated, interdependent team possessing absorptive capacity (Cohen \& Levinthal, 1990; Cunningham, 1994).

Team members are cognizant of the goal, or end, their work serves. They challenge and reflect on their practice by engaging in dialogue with other team members, customers, and managers (Dixon, 1998). And they actively evaluate their practice. The proposal team is proactively norming and performing as described in Tuckman's theory of group development.

In the forming phase, the group members become oriented toward one another. In the storming phase, conflicts surface in the group as members vie for status and the group sets its goals. These conflicts subside when the

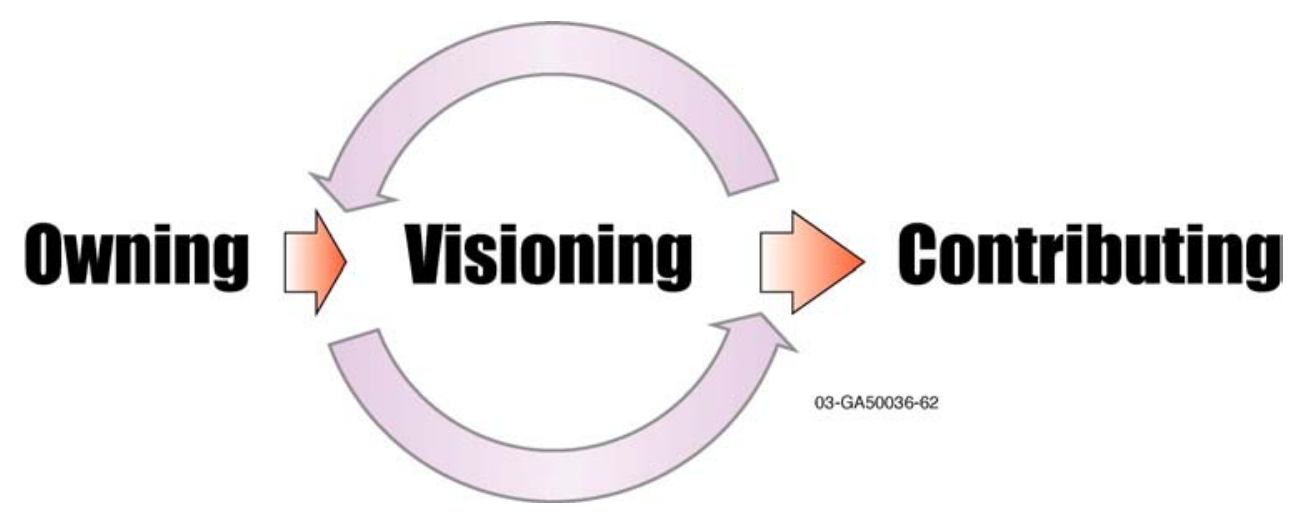

Figure 9. Homogeneous informants view of their proposal practice. 
group becomes more structured and standards emerge in the norming phase. In the performing phase, the group moves beyond disagreement and organizational matters to concentrate on the work to be done. The group continues to function at this stage until it reaches the adjourning stage, when it disbands. (Forsyth, 1999, p. 15)

Tuckman's final stage of adjourning does not occur at the end of each proposal process as the team moves directly to a new proposal preparation activity. The only adjourning activity that occurs is a team debrief with the managers and authors to establish ways to improve the process (Anfara, Brown, \& Mangione, 2002; Dixon, 2000; Capezio, 1996). The debrief process is valued by team members as they have an opportunity to dialogue with managers and authors about all steps of the proposal process. The outcome of the debrief is a norming activity to enhance their proposal practice. The norming activity could involve improving a guidance document, modifying the format of the author/technical reviewer checklist, or adding a running footer with the proposal title on each page. Each debrief builds team understanding of customer needs and deepens the team's absorptive capacity. Additionally, the debrief helps to move convergent knowledge to the core of the practice. Literature on team performance indicates that, "Project recaps are simple but powerful team motivators. It's vitally important for team members to see results. Seeing is motivating; keeping something visible keeps it in the forefront of thinking" (National Press Publications, 2001, p. 247).

The continual norming and performing steps allow the team members to become proficient and confident in the proposal process and to develop their capabilities to function in new proposal situations. Each new proposal cycle shortens the team's forming-storming phase, which is now a brainstorming process, allowing team members 
to spend more time norming and performing. Since the group is norming and performing aligned with a strategic business process, they are functioning as a community engaged in common practice working to make tacit practice knowledge explicit. Thus the team continually increases their capabilities both in the proposal practice and interfacing with customers, the team produces quality products more cost effectively and builds its future (Liedtka, 1999).

\section{$\underline{\text { Core Team Attributes of a Community of Practice }}$}

The proposal team's practice aligns with many of the attributes of a community of practice. A community of practice is social rather than an individual process where content is learned in the context of joint enterprise, called practice. A community of practice results in the generation of knowledge through active group participation. Learning results by engaging in social practice to negotiate meaning, by using imagination to see connections through time and space, and by aligning activities to broaden the enterprise (Wenger, 1998).

Because a community of practice is a living repository of community learning, knowledge is created, accumulated, stewarded, and diffused in the organization. Mutual engagement in the practice by community members allows negotiation of the explicit and tacit components of community knowledge. Communities of practice can vary in the extent to which they explicitly undertake the stewarding of knowledge for themselves and for the organization. Strategic learning communities of practice take responsibility for establishing and developing their practice and their community (Wenger, 2000a). The editors, graphic artists, and text processors work collaboratively to allow the author to focus on the scientific and technical content of the proposal. The proposal process establishes a new level of textural and graphical quality in proposals so reviewers connect 
with the author's concept and transmit a favorable recommendation to the funding agency. The benefits to the funding agency are articulated and aligned with the stated needs in the solicitation compelling the agency to fund the proposed scientific or engineering concepts.

To increase the win rate of proposals assisted by the proposal team, the members meet regularly to reflect on their practice and establish tools and practice enhancements to allow them to be more efficient. Debriefs with customers provide new insights into practice improvements. These reflective activities align with the community of practice approach to ensure that the community members are continually learning. For a community of practice to flourish, members must have a strong sense of belonging and engage in new learning initiatives to ensure the community's knowledge does not become stagnant. Members regularly reflect on their practice to keep from becoming captive to past practices. The negotiation of the meaning of knowledge in a community of practice results in members learning and transforming; thus, the current practice, the status quo, needs as much explanation as the need for change (Lave \& Wenger, 1991). Communities of practice engage in the "generative process of producing their own future" (Lave \& Wenger, 1991, pp. 57-58). The reflective coding matrix process concepts of owning, visioning, and contributing shown in Table 3 manifest the team's passion about their future.

To speed enculturation of new editors into the proposal service, mentor/intern relationships have been established. Interns work collaboratively with an assigned senior editor who mentors the junior editor by providing guidance, answering questions, and presenting opportunities for new learning aligned with the proposal practice. The mentor/intern interactions are fluid, as an intern usually must interact with several senior 
editors to complete a wide range of job assignments. Thus, the knowledge and expertise of a proposal community of practice resides within the group rather than within any one individual. The core group, the masters, establishes community's ideals, which are transferred to the newcomers by mentors. The newcomer, or the intern, learns through practice with an experienced craftsman, or mentor. Mentors facilitate the intern's enculturation process guiding their understanding of their role and identity while mastering skills in the workplace (Pratt, 1998). The learner acquires both explicit and tacit knowledge framed in the context of practice resulting in a learning experience actively linked with the application of transferred knowledge.

The core team constantly leverages knowledge from past proposal efforts to improve proposal tools. Collaboratively the team negotiates enhancements to proposal guidance, templates, checklists, and timelines so that proposal tools provide authors with explicit insights into the request for proposal document (Kent-Drury, 2000).

Redish (1995) validates this portion of the visioning process when she states, "Sometimes, in fact, the technical communicator adds value by realizing that the best solution to a communications problem is to not develop a document or to develop an entirely different type of document” (p. 33).

By developing and using the tools the editors become more competent at the proposal process. Team members continually reflect on their practice to increase the quality and speed of team services and to increase their technical knowledge when working with authors with science and engineering backgrounds. This interplay of competence and capability within the community results in incremental learning. This convergent learning flows to the core of the community building and refining practice knowledge (Wenger, 2000b). Also the joint enterprise nature of a community of practice 
creates a sense of accountability to the community's body of common knowledge (Dixon, 2000). Community members engage in dynamic knowledge exchanges and actively negotiate all aspects of the community's explicit and tacit knowledge. The community members interact to jointly solve problems, to assist members in expanding their practice knowledge, to create new knowledge through joint activities, to bind the members of the community with a common passion for a body of knowledge, and to develop a shared repertoire of resources.

Radically new insights are needed to create new opportunities. Breakthrough learning occurs at the boundaries between communities of practice. Wenger (2000b) provides insight into the concept of boundaries as they connect communities and offer learning opportunities. Boundary learning opportunities are different than those offered by a single discipline-focused community of practice.

Inside a community, learning takes place because competence and experience converge....At the boundaries, competence and experience tend to diverge: a boundary interaction is usually an experience of being exposed to a foreign competence....If competence and experience are too close, if they always match, not much learning is likely to take place. (p. 234)

The proposal process occurs at the boundary of many communities of practice-the soliciting agency, organization managers, scientists, and engineers. Proposal team members participate in boundary activities with each document effort as they collaboratively work with authors to produce a document. Wenger (1998) explains the cautions and values of boundary activities when he states, "Becoming a community of 
practice in its own right is a risk of boundary practices that may thwart their roles in creating connections-but this risk is also their potential" (p. 115).

At the boundaries, competence and experience tend to diverge as members are exposed to a foreign competence. In communities of practice, the value of the core and the boundaries are complementary. Deep technical expertise depends on the convergence between experience and competence, but innovative learning requires the divergence of experience and competence. However, balance between core and boundary-learning processes allows core practices to enable deep, discipline-linked learning while boundary practices result in knowledge production, exchange, and transformation. The divergence of competence and capabilities at boundaries results in innovation as team members are forced to reflect on assumptions. Boundary processes are a source of transformational learning, resulting in step changes in the community's body of knowledge. Thus, absorptive capacity is built at boundaries (Cohen \& Levinthal, 1990; Wenger, 1999, 2000b).

The evolution of the proposal guidance document exemplifies the potential of boundary processes. The first guidance document focused on format and simple text instructions. Debriefs revealed the need for more focused guidance instructions. Current guidance documents provide in-depth coverage of the call by blending

- $\quad$ proposal requirements taken directly from the call,

- $\quad$ insights about approaches to sell your idea, including examples of graphics that will focus section text, and

- writing techniques to achieve clarity, concision, relevance, and consistency, and by framing the guidance in a call conformal, visually appealing page layout. 
The proposal team uses input from participants gained through dialogue and debrief meetings to ensure the evolution of their practice. Based on this input, the team may enhance a process step, prepare an additional tool, or eliminate a step that adds little value to the process. The value of strategically enhancing the proposal practice allows the team to obtain an intellectual advantage. The balance between core and boundarylearning processes allows core practices to enable deep, discipline-linked learning while boundary practices result in knowledge production, exchange, and transformation. For an organization to develop a knowledge economy, informal interactions such as conversations, brainstorming, and pursuit of novel ideas are important. If communities of practice flourish, the informal community can drive strategy, generate new business lines, solve problems, promote new practices, capture lessons learned, develop member's professional skills, and assist in recruiting and retaining desired talent.

The proposal service informants' have affirmed their community of practice by owning, visioning, and contributing to enhance their practice. The reflective matrix (Table 3) provides the attributes of their community of practice. Their feelings of contributing reflect their perspective on their boundary practice as they enable others to achieve without personally receiving monetary benefit from a proposal win. This feeling is consistent with the way the team values their contributions because "the top two things that consistently motivate people are achievement and recognition" (National Press Publications, 2001). The process concepts of owning, visioning, and contributing reveal the team's dedication and passion about the future of their practice. Thus, the theoretical position emerging from meta-inquiry explores the evolution of the proposal team's community core and boundary practices. 


\section{Theoretical Position}

For organizations to obtain an intellectual advantage over their competition, there must be a learning culture that promotes regular interaction, mutual interest, recognition of the intrinsic value of learning, and incentives for sharing among members of the same community of practice and between communities of practice (Storck \& Hill, 2000). The consequence of team members' passion about owning, visioning, and contributing to their core practice is the basis for my theoretical position.

The theoretical position states that cross-disciplinary team members participating in a well-defined, business-critical process can make measurable contributions to the healthy future of an organization when participants have autonomy to own, vision, and contribute to their process practice. The adjective, cross disciplinary, is essential for the proposal practice as each core team member's discipline knowledge adds a unique facet to the structure, form, and unity of the final proposal product. The grand tour question that evolves from this theoretical position isCan a community of practice evolve and be cultivated within an organization to the strategic benefit of that organization?

To confirm that the grand tour question aligned with the core team's proposal culture, I used a member check process and shared the theoretical position and proposed grand tour question with the meta-inquiry informants to confirm the theoretical position and grand tour question capture their perceptions about the proposal practice (Anfara, Brown, \& Manigone, 2002; Creswell, 1994; Glaser, 1978; Guba \& Lincoln, 1981). Guba and Lincoln (1981) name the process phenomenon recognition for involving informants in obtaining confirmation that their shared experiences are captured. I dialogued with several team members about the theoretical position and grand tour question and the 
accuracy of reflecting their practice. The range of affirmation comments struck me. One team member provided an email confirming the theoretical position aligned with their perspective of the proposal practice. The team member wrote

"It's true that people care more about something that they've been able to put their own creative touch into. Also, it's a compliment to know that you're trusted enough to do what is requested without having someone constantly staring over your shoulder. It adds a sense of pride to your work."

Another team member recounted concern that the theoretical position was too obvious. This observation provided confirmation that meta-inquiry does increase the researcher's theoretical sensitivity to the human ecology under study.

The grand tour question resonated with core team members, and the meta-inquiry analysis and interpretation accurately framed their voice. Using the grand tour question validated by core team members, I developed a formal interview protocol and phrased questions to provide heterogeneous informants with the opportunity to affirm or refute the team's perception of their practice. 


\section{Chapter 5}

\section{$\underline{\text { Formal Interviews }}$}

The theoretical position and grand tour question emerged during meta-inquiry as I reflected on the core team data and developed interview questions for dialogue with heterogeneous informants. Because an effective interview protocol looks for similar as well as dissimilar data to foster concept exploration, the formal interview protocol builds from the grand tour question-Can a community of practice evolve and be cultivated within an organization to the strategic benefit of that organization?

Main questions posed to the heterogeneous informants probe customer's views of the proposal process. Norming questions are added to better interpret answers based on each informant's perceived knowledge of and comfort with proposal preparation (Glaser, 1992, 2001; Strauss \& Corbin, 1990).

The main questions for the formal interviews are

- What value did the proposal service add to your proposal?

- What value do you place on proposal service prepared tools like the guidance, template, and checklist?

- How could the service be improved to better assist you in the future?

- $\quad$ Based on your interactions with proposal team members, what skills and knowledge do you feel they contributed to your proposal? What skills and knowledge were lacking?

- What personal value do you place on working with the proposal service?

The norming questions are

- What is your usual approach when responding to a proposal opportunity?

- How comfortable are you with your normal proposal approach? 
These main and norming questions framed the first few interviews, but I modified questions as categories emerged. Glaser (2001) advocates this interview approach when he states:

GT [grounded theory] requires the freedom to interview in whatever style works at the moment or time in sampling for incidents to compare. The questions are content guided based on the emerging theory's categories as the research generates properties of them. Thus emergent questions are emergent interview guides to use on one or a few participants available at the time. Emergent interview questions are NOT to be used with all participants. The analysis of a few interviews will usually change the subsequent questions as the researcher samples for data in different aspects or directions. Much of the time the researcher is just listening in a kind of open-ended conversational interview. As analysis proceeds questions are formulated to help saturate categories. (pp. 174-175)

The grand tour question and formal interview protocol provide additional data for emergence of actionable theory. Constant comparison during open and reflective coding allows valuing of both the heterogeneous and homogeneous informant data to determine the categories and properties of the emergent theory.

Before starting formal interviews with the heterogeneous informants, I coded the minutes from twenty-six proposal debriefings. Using artifacts as my initial excursion into the heterogeneous informants culture aligns with the qualitative traditions of both ethnography and grounded theory (Creswell, 1998; Spradley, 1980; Wenger, 1998, 2000a). Eisner (2000) explains the value of using artifacts to gain understanding into the learning capacity of cultures. 
If culture is, as I have suggested, the most telling repository of human capacity, then I suggest that we inspect the culture to discover what might be called "cognitive artifacts" (the products of thought), that we use these products of thought to understand what we can of the forms of thinking that led to each, and that we try in the process to grasp the kind of meaning that each provides. (p. 249)

Debriefs occur after each major proposal effort resulting in the submittal of multiple proposals or a funding document involving multiple authors. The proposal team members, authors, and managers meet to critique the process. The debrief process is very interactive as authors, managers, and team members freely share their insights. Any informants unable to attend the debrief are invited to provide their insights via email. The meeting and email comments are combined and blend the voices of the homogeneous and heterogeneous informants as they review a unique proposal process. When the proposal effort involves only one author, either the proposal team leader or an editor, not involved with the proposal, contacts the author by phone to critique the process. All debrief minutes are stored in electronic and hard copy form in a working folder for each proposal. The proposal team uses debrief lessons learned to improve their practice so mistakes are not repeated (Cunningham, 1994; Dixon, 2000).

$\underline{\text { Researcher Reflections }}$

Before coding the debrief comments, the formal interview protocol contained no norming questions. Following the coding of the debrief comments, I reflected on the main questions and added norming questions. The additional questions probe the informant's usual proposal strategy. From the debrief process, I noticed a wide variation in authors' approaches to proposal preparation based on the complexity of their debrief 
comments. Some are quite capable when responding to a proposal call; others are bewildered and had no effective, logical strategy for proposal preparation. The data describing their strategy provides me with insights into their usual proposal approach so I can frame their responses based on proposal process knowledge. Dialogue with experienced proposal authors provides an opportunity to capture process enhancement ideas for the team to consider adding to their practice.

One recurring source of author concern relates to senior management providing timely funding support for proposal writing. There is a great deal of author frustration about the need for an organized management funding strategy to identify, endorse, and quickly fund the author's proposal preparation time on critical proposal opportunities.

Because the proposal team has established a proposal process and proactively works that process, we are the focus of praise as well as criticism. I welcome the criticism as it causes the team to reflect, but unfocused, general criticism received after a demanding proposal effort is difficult to capture and use to improve practice.

At debrief meetings I actively dialogue with authors and managers to obtain the details about their concerns. If they cannot explicitly explain their concerns by illustrating their concern with a specific example, I assume it is just an author venting their frustrations. It is critical that the research or engineering manager attend the debrief so they can assist in defusing a situation when the comments are especially hurtful so the proposal team receives useful, explicit input to improve their practice.

I used the meta-inquiry reflective coding matrix (shown in Table 3) for the open coding of debrief minutes. Prior to analysis of heterogeneous informant data, I had no basis to establish if the homogeneous reflective coding matrix was appropriate or inappropriate for coding heterogeneous data. 
Also, in discussion with the qualitative researcher providing an audit trail for research findings with potential political impact in regards to the laboratory, we decided to limit managers' interviews to only the research and engineering managers actively involved in proposal efforts (Guba \& Lincoln, 1981). Initially, I had anticipated interviewing vice presidents and associate laboratory directors, but we established they were too removed from the proposal process to provide data directly linked to proposal practice. The merit of this decision is confirmed by Senge (1990) when he says, "Top management teams are often removed from key operational policies and have less influence in creating change than widely assumed" (p. xix).

\section{Debrief Data Analysis and Interpretation}

The result of open and reflective coding of debrief minutes added the reviewing dimension to the visioning process and an additional, totally new concept, producing, to the meta-inquiry reflective coding matrix. Also, the strategy for understanding the consequences was modified to align with the debrief and homogeneous informant data. The modified strategy describes the strategic evolution of practice. The enhanced reflective coding matrix is shown in Table 4.

The reviewing dimension of visioning focuses on the internal technical review process, a required part of all proposal service led efforts. Although a review process is a facet of the editor culture discovered during topic-oriented ethnography, the proposal team is constantly reviewing its practice by working collaboratively with other team members and the author (Liedtka, 1999). Hughes (2002) states,

A knowledge-centered approach argues that technical communicators need to be brought in as soon as possible so they can facilitate the complex 
Table 4

Reflective coding matrix - homogeneous informant and debrief data

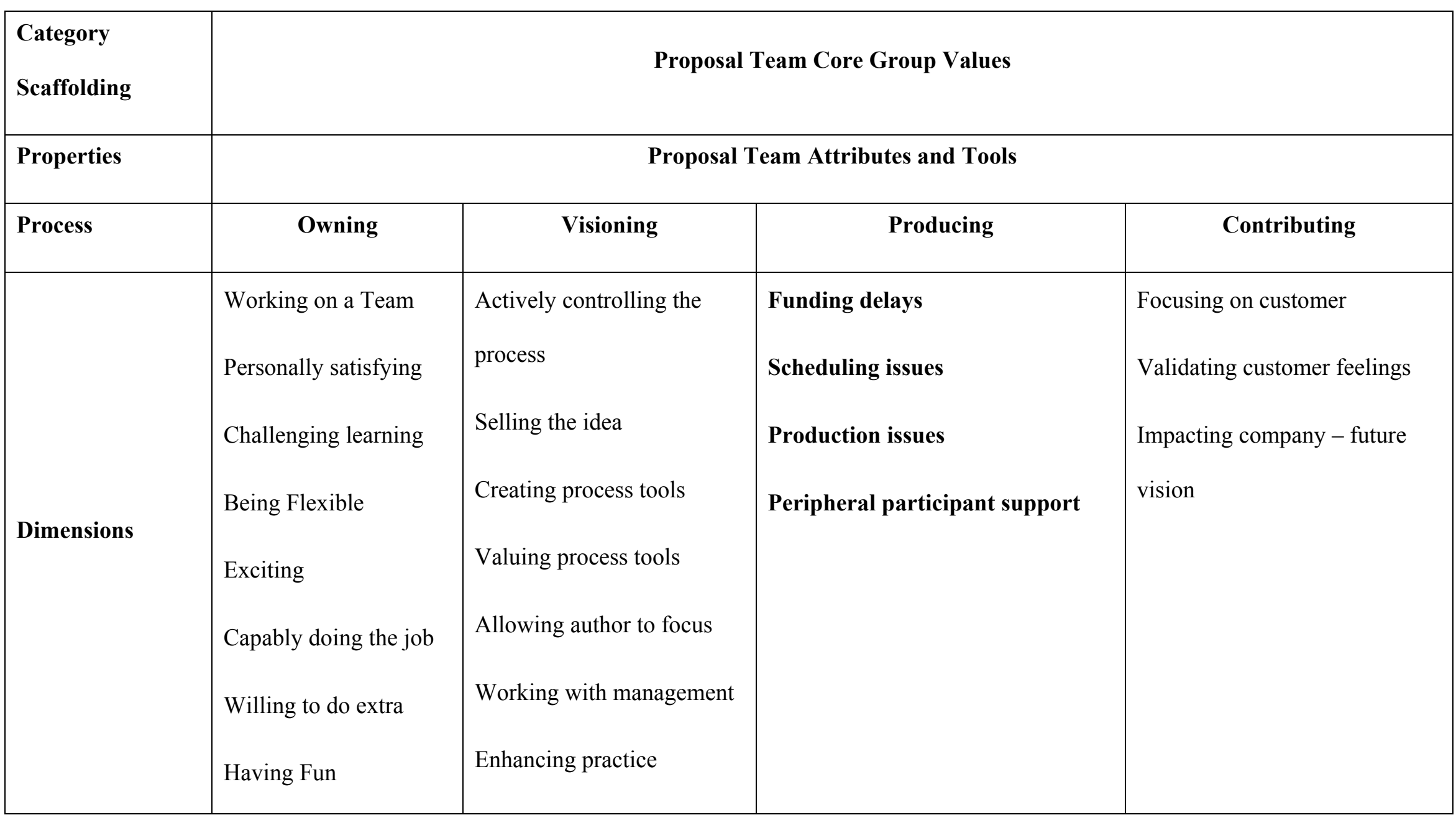




\begin{tabular}{|c|c|c|c|c|}
\hline $\begin{array}{l}\text { Category } \\
\text { Scaffolding }\end{array}$ & \multicolumn{4}{|c|}{ Proposal Team Core Group Values } \\
\hline Process & Owning & Visioning & Producing & Contributing \\
\hline $\begin{array}{l}\text { Strategies for } \\
\text { Understanding } \\
\text { the Consequences }\end{array}$ & \multicolumn{4}{|c|}{ Strategic Evolution of Practice } \\
\hline
\end{tabular}


process of knowledge creation and promotion. Rewrites are seen as the natural emergence of knowledge and, therefore, a source of value rather than inefficiency. (p. 284)

Thus, the activity of reviewing the proposal process did not emerge as a unique dimension during meta-inquiry using only core team member interview data. For team members, reviewing is linked with all the dimensions of the visioning process. They value the technical review process as a step that "technically strengthen[s] the proposal" and a way to enhance "solid science logic" in proposal rhetoric. Thus, the review provides the editor with knowledge to more capably do their job.

Authors value reviewing as a unique step in the proposal process. Reviewing steers the practice by enhancing the document and guides the author and editor to produce a technically sound, quality proposal. The review is revealing to the author at the personal level, not at the rhetoric level. The review validates the merit of their proposed science or engineering concept. Thus, the author has a professional stake in the outcome of the review process. Authors indicated the review process is a necessary step, but "the reviewer should want the proposal to succeed. Their comments should help it to succeed." Two authors shared their reflections on the review process. "I expected my paper to be butchered and wanted that type of review but did not receive it." And we want to "have our proposals come back to us loaded with red ink." Authors value reviewer comments that allow them to strengthen the proposal.

For the review process to enhance the quality of the proposal "a face-to-face debrief with the reviewer, PIs [authors], and editors follows the written [reviewer] comments." When the review process works, it results "in a strong proposal with solid 
science logic." Thus, the authors' input from debrief minutes added the dimension of reviewing to the process of visioning in the reflective coding matrix.

Authors also value review comments from the agency reviewers as a source of input to strengthen a future proposal. One author discussed their agency comments following an unsuccessful proposal. "We need to resubmit it. I think it is a great idea and I think we should clean it up. Well, if you look at the reviews, none of the reviews were bad. There were no comments in there saying, 'You should have changed this.' It was good. This next year might be a little bit better."

Three of the scientists interviewed have served as agency reviewers for proposal solicitations. Reviewers value clarity and logical presentation in proposals. One scientist stated,

"You get it arranged so that it looks good to a proposal reader, which is very important. I know it is because I review proposals all the time. And when things are just slapped together, they are hard to read. You kind of want to just put it down and say, 'Eh. Let's not fund this one. I don't care how good this idea is. This is way hard to read."

Team members view the proposal product as part of their visioning process because the goal of their practice IS to produce a quality document. Team members know that goal will be achieved. But the authors view the production of the document as a unique process in the proposal evolution. Coding debrief minutes added an additional process, producing, with the dimensions of

- $\quad$ Funding delays

- $\quad$ Scheduling issues

- $\quad$ Production issues 
- $\quad$ Peripheral participant support.

When funding approval delays the start of a proposal effort, one author explains the impact. "This isn't the fault of VPS (the proposal team), but the timeline on these proposals was overly short...Management needs to improve the process so that both the scientists and VPS have more time." Another states "senior managers make the decision of when to support a call; they need to know the importance of announcing it immediately." The result of a funding approval delay put another author and the proposal team in a "the time crunch... a result of his organization's slowness in getting mobilized."

Scheduling issues focus on all the demands placed on the author's time. Proposal writing can coincide with the "year-end...many people had other demands," "some of the team was working on a work plan...the work plan was the first priority," or "a time constraint... because people were also working on a critical operational deadline." Thus, the proposal team leader needs to schedule the "kick-off meeting... as soon as possible [after] the white paper down select was complete and the list of proposers was established." The proposal team process appears to ease schedule demands (Kent-Drury, 2000). One author states, the "schedule would have been problematic without the VPS [proposal] team. Not sure that the proposals would have gone out without their efforts."

Production issues are the most problematic step for an author doing a proposal as an individual without the proposal team assistance. While production is part of the editor, artist, and text processor's culture, it is a foreign competence to many authors. One author remarked on a particularly text processing intensive proposal effort that "not everyone realized that each financial page was a separate PDF [electronic file] that had to be pulled into one main document." When an author collaborates with different institutions, communication of proposal document needs to collaborators may not be 
clear. This lack of clear understanding can result in an author receiving forms of unacceptable quality, unresponsive text, poor quality graphics, incorrect budget pages, or incomplete references. The proposal team then faces production issues. For example, when "financial pages were not high quality" the text processors "enhanced the quality the best they could, but only so much could be done."

Also, authors have a hard time putting down their pens and allowing the proposal team to unite the document and give it final structure and form. As one proposal team member explains,

"At the end, there must be some time when the authors can no longer make changes to their sections and they hand it over to the editors. The editors, VPS team leader, graphic artists, and text processors must have time to do a final check before the document goes to printing."

To address the time constrained dimension of the proposal practice and the impact of any delay on final document production, the team works on approaches to use time more wisely. One strategy is a designated time each day-one hour-when all team members focus on documents exclusively. Limiting distractions allows the entire team to increase its productivity.

The cry of "never enough time" is always heard during production. The team always generates a completed document by the established deadline because they have "the knowledge of getting all that work done in that short period of time." To speed production of the final document, the team pushes to have a well-formatted draft for review. One manager states, "this proposal process pushed for such a well-done draft that it saved time in the final document." 
The value of peripheral participants' support surfaced in the debrief minutes. The team relies on the talents of the financial analysts to prepare the budget sheets, the print shop to produce a quality color document, managers to actively support author's and proposal team's needs, and administrative personnel from the author's work group to handle mailing issues. In the final proposal process steps, these peripheral participants are essential for completing production and transmitting the proposal documents to the funding agency. The financial analyst some times has a "hard time getting information from collaborators. The information often came in different [budget amount] than the PI [author] had assumed it would" so the analyst must work with the author and collaborator to reconcile the differences. The print shop produces the final hardcopy of the proposal. One author was "very happy with the quality of the final proposals...the print shop printed beautiful color copies." The manager and his support staff must transmit the final document to the funding agency. One secretary "picked up the copies at the print shop [in a different building] and mailed them. It went great!" In another effort the team "received excellent support from the technical manager and his administrative support in the mailing process."

The debrief data provided a clear indication that the homogeneous reflective coding matrix lacked the necessary robustness to capture the voices of heterogeneous informants. The minutes provided additional insight into the process dimensions of owning, visioning, and contributing and added the process of producing to the core team data. Coding the debrief minutes prior to conducting interviews with heterogeneous informants also increased my theoretical sensitivity to the human ecology of the different cultural disciplines of the scientist, engineer, and technical manager. 
Glaser (1978) clarifies the value of looking at varied informant data when he states, "The fullest possible development of formal categories, is achieved by comparing incidents or ideas from any group, irrespective of differences or similarities, as long as the data indicates one similar category or property" (p. 152). This approach moves the analysis focus from the individual informants to the basic social processes of the proposal practice. Substantive theory emergence depends on switching the researcher's 'focus from studying the unit to studying the process" (Glaser, 1978, p. 107).

I discovered that some of the processes that result in convergence of core team knowledge differed from the processes valued by communities of practice engaging in boundary activities with the proposal practice. For the team their proposal practice includes tacit knowledge about reviewing and producing while the same knowledge is viewed as explicit steps of the proposal process by the authors. The team's tacit knowledge must be made explicit and valued by the proposal team to allow all team members to appreciate customer feelings and interactions relating to the reviewing and producing aspects of proposal practice. Debrief minutes reveal the evolution of the proposal ecology by adding a new process to the proposal ecosystem.

\section{Topic-oriented Ethnography}

Formal heterogeneous informant interviews provided insights about other cultures' views of the proposal community of practice. Topic-oriented ethnography studies of the culture of scientists, engineers, research managers, and engineering managers completed before the grounded theory analysis and interpretation expanded my understanding of other communities of practice and the dynamics of boundary activities on the proposal practice. Wenger (1998) cautions about the value and danger of boundary activities to a community of practice when creating connections. 
As the proposal team has interacted with over 250 scientists, engineers, and managers, I initially interviewed research and engineering managers to determine the value they placed on the proposal practice. The managers' data directly link with the impact the proposal practice has both on company business goals and on the personal value of the practice to the managers (Bennett, Fadil, \& Greenwood, 1994). The manager data explore facets of the proposal practice that require enhancements to increase the proposal win rate as the managers dialogue with and receive feedback from the funding agencies or the federal field office on proposal documents.

\section{$\underline{\text { Technical Managers }}$}

Data from research and engineering managers were combined as their interview data revealed no differences in their perspectives of the proposal practice. Their interview data are presented as the topic-oriented ethnography of technical managers.

All the interviewed technical managers valued their interactions with the proposal team. Each manager had led a minimum of one proposal effort prior to working with our team. Most of the managers provided a clear insight into the complex process of preparing a proposal.

"Sit down, understand what the requirements are for the call itself... I am talking formatting and pages... all the administrative stuff....But my role has always been one of facilitating the proposals and...the job has been one of understanding what the needs areas are, what the call wants, and looking across the lab to bring together the technical teams that could be responsive to that."

Additionally, one of the managers had led a large multi-million dollar engineering proposal effort. In the engineering proposal the use of "theme sentences and themes... are 
the main things that you need to stress." Thus, technical managers' perspectives on the proposal practice are framed by personal knowledge of the complexity of the practice.

Managers focused on the need for quality proposal documents to support their group's business targets. In the past "success used to mean 'meet technical specifications on time and within cost', success now means 'do this in a way to satisfy all stakeholders"” (Kurstedt, Mallak, Howard, \& Kurstedt, 1990, p. 162). Interviewed managers are aware that the proposal must meet the stakeholders' requirements and must sell because

"If I say, okay, I am writing a proposal and there is a section on engineering and construction... Who is the customer when you are working on a proposal....They don't really care what your problems are, but here let me tell you about engineering."

Each manager is responsible for providing funding to the laboratory either from research funding agencies or from the federal field office that manages the laboratory. This responsibility is taken seriously, and the managers strive to leverage their limited proposal development funds to prepare winning proposals. One manager placed great value on the assistance of the proposal team in helping them to achieve a high measure of leverage with their preparation funds.

"I was very pleased to learn that we won four proposals with [the laboratory] as a lead and four proposals with other organizations as the lead. We won $\$ 5 \mathrm{M}$ over a three-year period! This is a factor of nearly $50[\%]$ return on investment! This was an outstanding effort with limited funding. [The proposal team] performed a lot of hard work supporting 
these proposals. I am sure that the [laboratory] received more funding than any other [proposing] organization."

Clearly understanding the exact needs of funding sources and regulatory agencies adds to the concerns and complexity of each manager's job. They strive to reduce the amount of rework by paying attention to completing the documents correctly and conformally the first time. One manager states,

"Yeah, but the formal submittal with printing and everything was like the 15 th of January. It was really about as fast as we could expect. And the other thing it did is it added the confidence of [the federal field office] that we really [could meet]...critical decisions. From what I gather from talking to other people, that isn't the way it always worked out for all the projects."

Also, regulatory agencies reviewed the same documents as the federal field office. Earlier funding documents to these agencies, prepared without proposal service assistance, were poorly received, but with proposal team assisting the engineering team, there was an incredible turnaround in acceptance of these complex documents. The agencies made few substantive comments on the documents. Other documents prepared since have been approved in a few days by regulators because the initial two documents helped to establish a level of credibility with the regulatory agencies.

Technical managers, who work with the proposal service, have many time demands in addition to managing proposal efforts. Managing a proposal process by themselves, without proposal service assistance, impacts their ability to devote time to other critical tasks. The proposal team provides the managers with a structured process and actively manages the process affording the manager time. On one proposal effort, an 
editor, using resource materials provided by the manager, prepared approximately $50 \%$ of the required proposal text. The manager confirmed the editor had prepared solid text that speeded the process.

Technical managers are generally culturally extraverted and very personable. They strive to support their employees with processes that enable them to successfully complete tasks and meet customer expectations. Using the proposal team requires "the knowledge...[of] when to use the service and what to do if you can't." Two managers have modeled parts of the proposal team process in their current business practices. One manager actively controls work group document processes through implementation of a customer expectation process by indicating the level of editing required on each document. He explains, "So now we have an expectations checklist that we fill out." Another manager requires his employees with weak writing skills to participate in the laboratory technical writing courses managed by the proposal team leader and taught by one of the team's editors.

Managing the entire proposal process and crafting text to sell a concept is sometimes a challenge for the managers. They are in their management positions based on their deep scientific, engineering, or program management knowledge and their ability to manage cross-disciplinary science or engineering teams, not their writing and artistic capabilities. They value the practice of the proposal team to sell the idea using text and graphics. All managers can appreciate the power of a clear, concise, textual message, but the proposal practice brings the power of graphics to the proposal. One manager shared his impressions of document graphics. "In the graphics area, that was just top notch." Additionally, the team coordinates the resources of the production staff that includes text processing and printing. "The knowledge of printing and what it would take to" do a 
quality printing job is the final step in producing an artifact managers are proud to submit for funding.

Submitted proposals, whether funded or not, articulate the technical capabilities of the laboratory. The documents establish the quality and credibility of the engineering or scientific concepts (Meade, 1998). These documented capabilities can result in additional funds for the laboratory as one manager explains,

"And we won, we won more than our fair share each time. So I think we have started to establish ourselves there. It certainly helps, it helps me, when I go out to essentially market this laboratory's capabilities, if I go to one of the closure sites or if I go back to headquarters... it is quite easy to state and defend our ability to adapt and deploy advanced technological approaches for clean-ups and that we have a successful track record of doing it."

Another manager credits the proposal practice with assisting him to be successful on a highly visible document series that was critical to his project, his employees, and the laboratory.

Over the last three years, the team has worked repeatedly with some of the same managers so the managers have developed an appreciation and understanding of the proposal practice. Each manager is passionate about their desire to enable their employees to achieve. The power of this leadership and concern for their employees is appreciated by most of their employees. Research has shown that managers like these who lead by example enable their employees to achieve success and job satisfaction (Becker, Billings, Eveleth, \& Gilbert, 1996; Lencioni, 2000). Also, the managers interviewed value the proposal practice's ability to enable their employees to successfully 
compete for funds. They value the editing, graphics, and production coordination as well as the finished product. One manager recalled a proposal cycle before our service.

"When we did the first round of [proposals]-it was before you were invented, which was unfortunate, we actually did set up a war room and functioned and performed in many of the same ways that technically you guys function now."

The managers freely offer suggestions for enhancements to the proposal practice and proactively work with the proposal service alerting us of upcoming proposal opportunities. This allows the team to develop a resource strategy and prepare proposal tools in a timely manner to support the proposal effort.

They empathize with the proposal team members and value the team practice even when some authors undervalue our proposal practice. Henry (1998) explains the undervaluing when he states,

I believe that models of singular authorship are part of the problem in adequately representing the value that technical communicators add to an organization's products: when seen as wordsmiths who package other people's content rather than as professionals who tap many kinds of expertise to yield better content, communicators' 'added value' is typically underestimated. (p. 207)

\section{$\underline{\text { Engineers }}$}

Kurstedt, Mallak, Howard, and Kurstedt (1990) indicated that, "Traditionally, many engineers chose their profession because they wanted to deal more with structured machines, materials, and processes, logically related through drawings, scientific laws, and mathematical formulae" (p. 159). Attributes of an engineering culture include a 
short-term, practical focus, manipulation of objects, deference to managerial and social authority, use of quantitative rigor and rational-deductive logic, selection of useful, practical approaches, efficient use of resources, compliance with the status quo, and a tendency towards standardization and uniformity (McIsaac \& Morey, 1998). The engineer informants demonstrated many of these cultural attributes.

Engineers interviewed have a broad knowledge of many allied engineering disciplines, and they realize the value of other disciplines to complete an assignment even though extant literature indicates that different specialties in engineering normally do not value the contributions of others (McIsaac \& Morey, 1998). A single engineer does not have the detailed knowledge to complete all phases of the project. For example,

"An engineer, you know, mechanicals are working with electricals to make sure everything gets installed and hooked up right. They are working with civils to make sure they get the foundation poured right. And they know they are turning it over to the construction team, and it might go out on the street and get bid. And so we know that there is a whole slew of people involved with using our product to get something built and turned over to the customer."

They are product-focused and must provide a customer with a product on a set timeline. To produce the product, they explore known technologies and design components only if they cannot be procured. The customer is their focus.

"An engineer is, in my world, probably broader. And they are aware of, they are in tune with, they are very aware of the fact that they have to get something built and turned over to a customer in short order. Their knowledge base is based on knowing a lot of stuff and knowing what 
commercial products are available to meet the need and then getting those installed into a system to keep the customer happy, in short order."

To achieve success on an engineered product, they need to work collaboratively with other engineers, designers, procurement agents, and drafters; thus, engineers are oriented towards teaming with others to achieve success. The teaming is formalized in their work processes as one engineer explains,

"You know, it is easy in the engineering directorate because we know we are going to have a project engineer on every job. We are kind of formalized. We have, there is a project manager working with the project engineer [PE], and then we'll have discipline-specific engineers working underneath them, but the PE is responsible for the technical coordination of stuff. So, we kind of have a focal point."

There is a wide variation in their comfort and proficiencies with writing, and writing is not a passion with most engineers. They are responsible for, and many prefer doing, hands-on projects. One design engineer explains it this way,

"So, they are not asking us to do new and innovative things. They are asking us to create a product to meet their needs. Granted, you might have to do some new and innovative things in it, but generally, a design engineer is more attuned to realizing that that product has got to get out and get built by others."

Because they must design engineered systems, most engineers can visualize components and either draw the component themselves or provide an artist with a sketch or solid guidance to render a drawing of the component. One engineer explained how the graphical approach to a document worked for him. 
"Well, I think the way, what we did there is we did drawings and then, sketches, and then we patterned the whole [document] around a set of drawings instead of starting out writing and having a few drawings, you know. So it pretty much turned us around. It was graphics-oriented. Because once you got the figures there, all you had to do was go through and explain the figures, which, it was easy to figure out what you were going to write next because you were just explaining the figures. If you had the figures in the right order."

The same engineer shared the challenges he faces when he is not writing to graphics in a similar document. "And when you just start out normally to write one, you kind of, it's hard; you are hard pressed to know exactly where to start and exactly where to go." So he saw real value in working with the proposal team because we simplified the writing task for him.

To complete their tasks, engineers appear to be comfortable talking with a wide range of support people with various educational levels. They also value using an established work process; providing the process makes sense to them. One engineer provides his feelings on the proposal practice process.

"Well, I guess the first thing, if I already knew the process like I do now, I wouldn't say to change anything, but starting out to work with the process without knowing what it was, I guess I would emphasize that more. Just say right up front that we're going to get a bunch of figures that explain the whole."

The engineer also liked the approach of storyboarding his section prior to discussing graphics with the artist and text with the editor. The storyboard allowed the section to be 
developed in outline and focused the effort because, "Well, just, like I said, I think the storyboards...all we had to do was throw a few words with the figures."

Several engineering documents for construction activities follow a preparation process similar to the proposal team's template and guidance approach. Explaining the value of organized document processes with writing tools to aid preparation of a range of documents, one engineer who writes construction documents put it this way,

"The majority of the work I see is construction specs that follow the ISC or the standard for construction, so those are all different. Yeah, we follow the format. We have, I can't remember, IS or ICS standards that we pattern our guide specs after. And so those are all pretty established. We have boilerplates for them."

Engineers work well with the proposal team and appreciate the process nature of the proposal practice. Several engineers who have worked with the proposal service have strongly recommended to their team leaders and managers that the proposal service should be used on their document because we add value and make the document reader-friendly. Based on proposal team interactions with engineering managers and engineers, our service is working with engineering to prepare guidance and template documents for recursive procurement documents, conceptual design reports, and project execution plans. As we prepare these writing tools, the engineers work collaboratively with the team to ensure the tools meet engineering needs.

\section{$\underline{\text { Scientists }}$}

The passion for science and research is evident in top performing scientists at the laboratory. They are devoted to the science knowledge in their discipline. Their singular focus can result in what appears to be uncaring behavior as they dismiss comments from 
people educated in fields other than theirs. They strongly advocate their research and stand their ground when others try to derail their research approach. As one scientist recounts, "I said, 'No, it is not a subsurface proposal with a little bit of corrosion. It is a corrosion study that tests subsurface environments."”

Because research funds are generally obtained by a proposal process, there is a wide range of scientist comfort with the proposal process. One scientist, who had little confidence in their usual proposal writing approach, stated, "Until I had worked with the Virtual Proposal Service [proposal service], I would say [I was] very, very uncomfortable" writing proposals. While other scientists place less value on the proposal service as they are, or feel they are, excellent proposal writers already.

Generally, research scientists undervalue the talents of anyone outside of their discipline focus because "of course it will always be nicer if they had more of a scientific background in your area." This is evident in author-team interactions as editors find some scientists unwilling to listen to suggestions about potential approaches to enhance their proposal's readability and sales orientation. Forrester (1965) points to a potential reason for this undervaluing when he states,

Engineers [and scientists] early see the importance of science but they may be well launched on their professional careers before they see reason to understand psychology, the dynamics of industrial systems, law, or even effective writing (p. 16).

But other scientists, who have developed an awareness of the contributions of other disciplines to the science enterprise, see value in the proposal practice and state, "you're underrated. You are a triangle team, if you will. You have got a lot of peripherals...you have got all the ones in the background that you don't really see." Another scientist who 
has worked with the proposal team on three separate proposals indicated that each proposal involved a different editor. Although each editor had a different approach to dialogue about the proposal concept and setting up the story line, the result on all three documents was always a good proposal.

Some interviewed scientists valued the proposal service tools because "that rigor is already done for you and you can work on getting the words right." Others value the process because "it is set up. You step through the process. I think part of our success is we don't have to worry about what the format was or who is going to do the graphics." A scientist indicated that the guidance tool saved time because when a question arose all the information was organized by sections for easy reference rather than trying to find information by searching the call language. Another scientist explained the value they place on the time saved by the proposal tools and services.

"Actually it was nice having someone do that for you so you didn't have to put a lot of time into it. It takes a lot of time and effort. And the other thing was the graphics and figures and all of that stuff. You know, on [the proposal], they put together the Gantt charts. It is not a trivial matter to put those things together and make them look good. I mean, it is a lot of work and that in and of itself kind of frees you up to think about more of the technical details. The help with formatting and semantics and that sort of thing is really nice."

A scientist has a deep understanding of a focused body of scientific knowledge, but may not have a breadth of knowledge in many allied disciplines. One scientist echoed this cultural attribute when they explained, 
"I think most scientists are myopic. They are so close to the work that they are doing; they forget that the audience isn't in their field of expertise most of the time. They forget how to be a layperson and talk to a layperson about whatever topic it is. We have a real bad tendency here to speak acronyms. So, we lose people. I mean, all the time, we lose people.” Working at the research and engineering laboratory, a scientist is an organizational scientist not a pure research scientist (Glaser, 1964). Thus, the scientist must advocate research deemed of value to the laboratory to achieve desired research facilities. Failure to produce products of value, generally winning new research funds, places the scientist's capabilities under scrutiny by peers and managers. To achieve the desired level of success requires the scientist to sell their idea. Some enjoy the selling process,

"I have to do it in a very gentle way, normally. And that is not how you write proposals. So, that was a real good thing that I had to learn how to strengthen my writing to a proposal form so that it would beat them over the head and get their attention. That was what the tech writer said, 'You have got to get their attention. If you don't get their attention, there is no point to your proposal."”

Scientists value peer validation. A solid technical review by their peers adds value to their proposal because if "you don't get the feedback from the technical review, you can't improve your proposal." To drive the quality of technical reviews, a group of scientists established criteria for an acceptable technical review. This group captured their criteria in a letter and the team transmits the letter to all reviewers. This letter resulting from group discussions among scientists provides insight into their expectations of an 
impactful technical review. The letter poses questions for the reviewer to reflect on and guides their review to ensure it is "objective, critical, and constructive." A few of the points in the letter are provided to illustrate the value scientists place on peer review, and the input they seek from a review.

- "Does the proposal contain compelling arguments that make the need for the proposed research clear? If not, how could the PIs enhance the impact of their proposal?

- Is the proposed research of sufficient scientific importance, and is the research likely to be of high quality?

- Is the scientific background for the proposed research presented in a clear and concise manner? Are there important gaps in the background material that might lead a reviewer to question the qualifications of the PIs? Does the presentation of the background material leave the impression that the PIs are in complete command of the relevant science?

- Is the proposal sufficiently relevant to the call? If not, how could the proposal be modified to make it relevant?

- $\quad$ Are their any 'gaffes' (budget divided unequally between the PIs, excessive travel funds, frivolous or inappropriate statements...)?

- Is the work plan too narrow or too broad?"

The review letter is included because it is a scientist artifact that exemplifies the scientist's valuing of the review process.

The proposal team, especially the editor, provides the scientist with another level of review by a layperson because "having an impartial person helping you do the writing or looking over your writing, even if they are just basically structuring it, you get that impartiality. You get that distance and that is exactly what you need to put something 
together so it makes sense to the reviewer." Another benefit of the editor as a reviewer is “to have someone step back and take a look at it who hasn't been involved in it from the very infancy or beginning of this idea." Hughes (2002) affirms the scientist's valuing of the editor as an impartial reader who uncovers areas of unclear language.

Of all the customers that the proposal team interfaces with, the scientist proves to be the most unpredictable. While most scientists at the laboratory see value in the proposal practice, some strongly voice their displeasure about having to work with non-scientists in articulating and illustrating their proposals. But this is the nature of the scientist's culture (Glaser, 1964; Merton, 1957; Polanyi, 1983). The scientist has a positivist view of one reality-theirs-and that singular view of reality pervades their interactions with others and makes working with them on proposals a challenge. This challenge provides the proposal team with opportunities to reflect on their practice and see their practice from the perspective of the scientist (Hughes, 2002).

The value of the discrepant informant voice is explained by Lawrence-Lightfoot and Davis (1997) as they reveal facets of portraiture.

In portraiture, we refer to this perspective that deviates from the norm as 'the deviant voice,' and we never stop listening for it, even as we become increasingly focused in our inquiry and certain in our analysis. The deviant voice is useful in drawing important contrasts with the norm; the divergence in perspective and the idiosyncratic stance helps us see the quality and contours of the convergent themes more clearly. The deviant voice is also useful in encouraging the skeptical, counterintuitive stance that the researcher must maintain throughout the course of the research. (p. 193) 
Teaming is not highly valued by many scientists because "well, with scientists, there usually is no team. Usually it is an individual with a few lab techs working to publish a paper or something." Because working with a team is not valued in the scientist culture, scientists can be introverted or extroverted. The most challenging team interactions involve the introverted scientist. With the introvert, the editor must develop an interview and collaborative working style to gain shared knowledge of scientist's proposal concept. Because the team must articulate the scientist's reality, dialogue is vital to understand that reality. Team members must be patient and persistent to uncover and understand the scientist's reality, as the scientist desires to articulate it in a proposal. This dialogue is part of making the tacit concept of the scientist explicit (Hughes, 2002).

During interviews, a few scientists did place value on working with an interdisciplinary team. Recently, more research grant language advocates the use of a collaborative, cross-disciplinary research team approach to solve intractable research problems. One scientist saw the need for a big team approach because the talents of many competent people are needed for proposal success, and the collaboration of several disciplines contributes to a winning concept.

A few scientists were concerned about the science knowledge of their managers, as the managers are not perceived as active scientists. One scientist said, "We need collaborative leaders not managers." Also, there is a need for managers to concentrate on mentoring a focused group of good scientists rather than the approach of trying to make everybody a scientist (Drucker, 1999). Aligned with this view of managers, one scientist shared their experience on a recent proposal process not handled by the proposal service. They indicated the manager running that process was little help to the scientist in the area of the science or the customer politics (Keller, 1992). Basically the manager is a nice guy 
but viewed as ineffective. Thus, some scientists do not easily embrace the view of a proposal process and its constraints. This scientist's reality of the manager as a leader is contrary to one article in the extant literature. Keller (1992) states, "transformational leadership would most likely appeal to employees who are well educated and who desire challenging work that can enhance professional growth and development” (p. 498).

Several of the organizational scientists feel that they are not receiving adequate science guidance from their managers. This view of management can impact the proposal service when we assist scientists who view the technical manager for the proposal effort as ineffective. In that situation, the proposal team may also be viewed as ineffective due to the association of the team with the technical manager. Theoretical scientists focusing their research on basic science proposals most often express this concern.

$\underline{\text { Researcher Reflections }}$

Writing the topic-oriented ethnography section on scientists proved to be the most difficult for me. Trying to make the scientist's culture explicit forced me to reflect on all the tacit rules in that culture-a culture of which I am a member. The fact that "scientists [have] their heads in the clouds" allows for amazing discoveries (Wolff, 1991). But the scientists at the laboratory are not pure research scientists who have unlimited budgets to explore; they are organizational scientists with yearly or bi-yearly deliverables-a science product such as a publication, a winning proposal, patent application, or invited presentation. Thus, competition is intense and rivalry to win increases the level of challenge the proposal team faces when working with some scientists. 


\section{Topic-oriented Ethnography Summary of Heterogeneous Informant}

\section{$\underline{\text { Interviews }}$}

When creating an aesthetic whole, the integration of concept, structure, form, and unity, the author provides the concept (Lawrence-Lightfoot \& Davis, 1997). The concept, an innovative and compelling research idea or engineering approach, is the basis for the proposal. To develop the concept one author explained, "What happens is I will be doing the work and then an answer will come out. And then I say, 'What if I take this and I turn it the other way'." The concept develops further because the researcher "Start[s] thinking about these things. We don't go home and have Einsteinian-type ideas shoot into my head. What it is is a culmination of a bunch of little things that come together, which I think is typical of most researchers, actually. And pretty soon you have got a bunch together. It is a culmination of a couple of ideas and saying, 'Okay. What if we combine them together and try it this way.' And we did the initial calculations because we had to show something, you know. We are not just pulling something out of the air. It looks like it works really well and you put them together... and you get a few proposals."

The manager, engineer, and scientist cultures each provide new facets to the proposal practice ecology. The interactions with these three cultures constitute the proposal team's boundary activities. The engineer and manager culture are most aligned ontologically with the proposal practice as these cultures are focused on striving to achieve a measurable outcome in a collaborative manner (Creswell, 1998; Guba \& Lincoln, 1981; Polanyi, 1983; Tashakkori \& Teddlie, 1998). Also, managers and engineers believe in multiple realities and are looking for ways to improve processes. 
They freely seek and incorporate good practices from others to improve their final product. The improvement can be energy savings, cost savings, time savings, increased functionality, or customer confidence. They view teaming as critical for building a shared reality and focusing on group success. Creation of a definable whole is a clearly understood objective by managers, engineers, and the proposal team; thus, their collective view of reality aligns with the constructivists' view and is positioned to the right on the ontological continuum shown in Figure 10. Wick (2000) confirms the constructivist approach aligns when working with others as "technical communicators are especially skilled at working with engineers and technologists" (p. 525).

Scientists are positivists striving to perfect self-defined reality; their research focus is that single reality. Those adding value to their reality are viewed as worthwhile and those perceived as drawing away resources or distracting them from their reality are viewed as worthless. There is a singular focus on their fiercely guarded research idea. Working with others in pursuit of their research goal is not viewed as a reasonable option because a level of control may be lost with such an alliance. But scientists seek confirmation from their peers that their research pursuit, their reality, has merit. Criticism is not accepted graciously from those deemed to be of lower status than the scientist or from an unfavorable review. The scientist culture is a unique culture of egocentrism, myopia, focused passion, and enthusiasm (Glaser, 1964; Merton, 1957; Polanyi, 1983). 


\section{Theoretical \\ Scientist}

Positivist

(One reality)

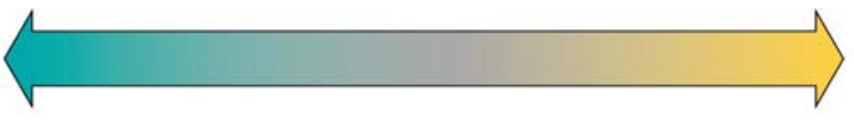

Applied

Engineer

Constructivist

(Multiple Realities)

Figure 10. Ontological continuum positioned in a science and engineering culture.

Proposal service alliances with scientists result in either complete satisfaction or dissatisfaction. When satisfied, the scientist actively advocates for inclusion of the proposal team support on their proposal efforts. Many times they specifically request an editor who has worked well with them in the past. If the editor is allocated to another proposal, there is hesitation to use a different proposal service editor; as to do so is to accept a change to their reality.

Boundary activities with scientists provide the most disconfirming insight into the proposal practice and, at the same time, provide the proposal team with opportunities to reflect on practice and implement step changes in their practice. The disconfirming information also holds great value to build grounded theory. Creswell (1998) explains, "The authors [grounded theory researchers] use rigorous procedures, such as collaboration and the search for disconfirming evidence, to verify their account" (p. 34).

\section{$\underline{\text { Coding Process }}$}

Because an effective interview protocol strives to reveal similar as well as dissimilar data to foster concept exploration, the formal interview protocol with heterogeneous informants included additional questions to explore barriers as well as the values of the proposal process (Boyatzis, 1998; Creswell, 1998; Denzin, 1997; Dixon, 1998; Glaser, 1978, 1992; Guba \& Lincoln, 1981; Patton, 1987; Rubin \& Rubin, 1995). 
During the fourteen formal interviews, I modified and enhanced the interview questions based on the dialogue with each informant.

The tradition of grounded theory as discovered by Glaser and Strauss (1967) provided my framework for open and reflective coding and constant comparison of homogeneous and heterogeneous informant data as well as for establishing core relevance. Glaser (1978) provides insight into the deductive and inductive nature of the coding process when he states,

When the grounded theory approach is used the researcher constructs his theoretical framework out of the data. Through comparing the data as it is collected, the researcher creates more abstract levels of theoretical connections. In short, theory is gradually built up inductively from the progressive stages of analysis of the data. (p. 39)

This level of theoretical abstraction allows core relevance to emerge. Krassen-Maxwell (1996) explains the importance of decoupling the data from the informants for the researcher to discover core relevance.

Shifting attention to conceptual categories and away from 'population' units, is crucial to the process of theory development, allowing the researcher to make comparisons that would otherwise not be possible. It places emphasis on the dynamic relationships between properties of the social process being studied, rather than on the static properties of norms and modal types. (p. 57)

Valuing all interview data obtained from a heterogeneous informant pool, comparative analysis provided the opportunity to explore, discover, and connect the research data and to gain increased theoretical sensitivity to the intricate relationships 
within the human ecology of the proposal practice (Glaser, 1978). During comparative analysis all informant data are correct and valued. Palmer (1998) discusses the subject of correctness when he states, “The subject itself corrects us, resisting our false framings with the strength of its own identity, refusing to be reduced to our self-certain ways of naming its otherness" (p. 106). Concepts are now developed using the homogeneous informant interviews, debrief minutes, and the heterogeneous informant interviews. These multiple sources of data allow the use of triangulation to blend the voices of the informants and neutralize bias adding validity and reliability to the research (Anfara, Brown, \& Mangione, 2002; Ford, Voyer, \& Wilkinson, 2000; Lawrence-Lightfoot \& Davis, 1997; Patton, 1987).

Strauss and Corbin (1990) motivate the act of concept ordering as necessary to build rather than test theory; provide researchers with analytic tools for handling masses of raw data; help analysts to consider alternative phenomenological meanings; cultivate simultaneous systematic and creative thinking; and identify, develop, and relate concepts, the building blocks of theory. Coding starts with all the informants' words that convey an experience, sensation, emotion, or mental image related to the studied social process. Grounding concepts in the data ensure fit, relevance, and workability (Glaser, 2001).

Early in open coding of the heterogeneous informant data, it became clear that the reflective coding matrix used with the homogenous informants needed to be enhanced to include the voices of the new informants as well as the debrief data. With the addition of the heterogeneous informants, the dynamics of the boundary interactions between human ecosystems involved in the proposal practice came into focus. The initial scaffolding category gave way to a more comprehensive, dynamic category, evolution of the proposal practice. The properties now explore the attributes of the proposal practice 
ecosystem. The processes of owning, visioning, producing, and contributing are expanded to include reviewing as a discrete process with the dimensions of importance, accuracy, qualifications, presentation, and benefit. The context expands to include the cross-disciplinary team developing a conformal, responsive proposal or critical funding document in a research and development and applied engineering laboratory. The strategies for understanding the consequences link to the ability of the proposal practice to flourish and adapt. Table 5 shows the reflective matrix used to value both the homogeneous and heterogeneous informant data.

The heterogeneous informant data provides richness to the research data tapestry based on the discovered threads of the scientist, engineer, and manager culture. Proposal service customers own, vision, and review their concepts to contribute to the future of the laboratory. One author, who freely admitted they love to write proposals because of the intellectual challenge of orchestrating the science logic and the ability to gather and explain the team's collective thoughts, reveals the excitement of owning the concept development process. The producing process is viewed as a separate mechanical process that is not cognitive.

Owning

The scientist's owning process is different from the proposal team's perception of owning, as the author owns a tacit idea that must be made explicit (Dixon, 2000; Hughes, 2002; Polanyi, 1983; Storck \& Hill, 2000). Working to make a theoretical scientist's concept explicit is a challenge. One scientist explained the abstraction of a theoretical concept using the word sphere. In that scientist's paradigm the word sphere brings forth a mathematical equation, not a graphical image of the shape. For the editor to convey the 
scientist's concept of sphere, they must take a mathematical concept and give it structure and form. 
Table 5

Reflective coding matrix of all informant data

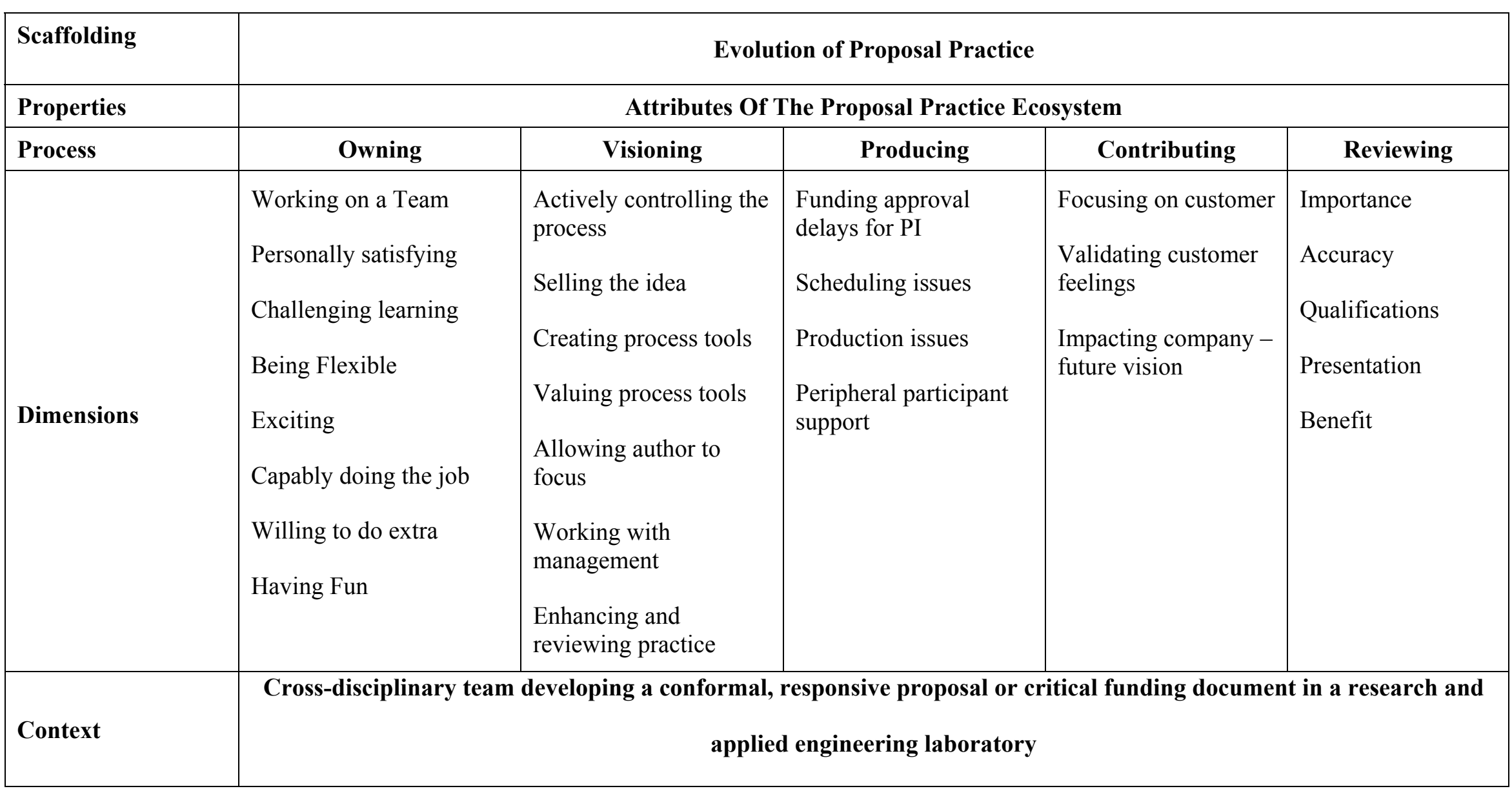


Another facet of owning discovered in the heterogeneous data is the maturation time for a concept to develop. The concept may mature slowly as this scientist explains, "How did I pull that off?... It was mine to begin with-whole heart and soul. I had been trying for six years." The same author was willing to do what it takes to put the concept on paper.

"And there were some mistakes made along the way. Looking back there are things that I would change in a heartbeat. Just because you see it now that you didn't see when you were, at two o'clock in the morning, trying to figure out why this doesn't sound right. So, there is a lot of effort there and I hope nobody has to go through that again."

For scientists challenging learning was related more to the process of understanding the audience for the proposal concept than the scientific process of discovering the proposal concept. One author carefully researched the need for the science concept.

"I did my homework and found out what they needed. I mean, I read their needs document from front to back cover in the proposal. The proposal is basically, many of the sections are a reiteration of their own words. Okay, what is stronger than your own words back at you? Okay, so that helped. And so I understood what their needs were. I understood the problem." Owning of a concept differs for the engineer and the scientist as one engineer explained,

"They are focused on such a different end product. Because a design engineer, a good design engineer, is excited if he finds a piece of equipment that will do what the customer wants and all he's got to do is 
install it. Because that makes his job easier, quicker, and he can move on to the next job... and a scientist, he wants to design that whole thing....He wants that ownership of saying they created it."

\section{Visioning}

Part of the visioning process for the scientist and the engineer involves selling their idea. One scientist stated, "That was the difference between getting the thing and not getting the thing and I'm convinced...It was all sales. I never realized. I never even thought about it really, but that is what you've got to do."

To sell the concept, the proposal tools suggest a strategy to present a solid abstract. One author explains,

"The abstract really sets the tone. You [proposal service] said it in the documents, 'You guys have got to make this abstract look good and make it so that people understand right at the beginning what you are trying to do.' You don't want to reviewer looking at it and saying, 'Boring or I don't understand a thing they are saying in here. This proposal is just excruciating to go through."

A scientist shares how he dialogued with municipal utility collaborator about selling a research grant idea. The scientist had previously worked with us on a winning proposal and learned the magic of the rhetoric process.

"We're trying to write a proposal without a request for proposal. This is something that you put together to the city-you turn it into the [federal agency] and you get a grant back. No format. No directions. No guidance. Nothing on what to do. It's like a shot in the dark. And they are writing this huge 10-page document, 20-page document-like a full-blown 
proposal. And I said, hey. Let's just put together a budget and say how much it will cost to do two years worth of work and like an abstract. Nothing but sales pitch high and go out and see these guys. If they want a full proposal, if they like what you're selling in those few words, for those dollar amounts, they'll come back and tell you what kind of format they want. They may not want anymore. They may just bring you a satchel of money and say here. Go forth. But, instead of trying to write this huge detailed proposal, it is easier just to write a few page sales pitch and all the essence is in the abstract. Look at it from the standpoint of an abstract." Not all critical funding documents are prepared by the proposal service due to timing and staffing issues. One engineer indicated that the proposal service tools and process are "real important to learn, if you're going to do it that way, we had to have your knowledge to learn what we were doing....I am sure there are still a lot [documents] around here that aren't written that way."

Reviewing

Two aspects of reviewing emerge. The homogeneous informants incorporate reviewing into enhancing practice as part of their visioning process. For scientists, reviewing is a distinct process. The importance of the review is captured in the introductory paragraph of the letter to reviewers developed by several laboratory senior scientists.

"The primary objective of the review is to help the [author] prepare a more competitive proposal. The review will also be used to help the laboratory focus its resources on those proposals that are most likely to succeed. To achieve these objective your review should be objective, critical and 
constructive. The internal review of proposals that seek to capture external funding is an important contribution to the success of the laboratory, and every member of our scientific staff is expected to carryout this task in a conscientious and thorough manner."

\section{Contributing}

The informants also talked about their approach to contributing. One scientist explained there are two perspectives to use when reading a potential call to ensure that the laboratory gains recognition from responding with a proposal.

- $\quad$ "Is the research opportunity one that will enable a cohesive research thrust in the next three to five years?

- $\quad$ Does the call fit within the science mission and vision of the group and will the research leverage interdependence among a multi-disciplinary team?"

A scientist with the example of an unallowable word illustrated the contributing dimension of focusing on the customer. For a specific basic science proposal the program sponsor does not tolerate the unallowable word-process. The sponsor had indicated in the past if you propose a process then you must have done the basic research or you would not have a process; therefore, he will not fund a process.

The proposal must contribute to the needs of the program sponsor to receive the opportunity of being technically reviewed. "Anything to help with that setup is really important to me. That stuff is like half the battle and knowing who your audience is and who the gatekeepers are and what they want."

\section{$\underline{\text { Ontological Considerations }}$}

Preliminary conceptual ordering of the data resulted in the interpretation that there is a textual and graphical continuum from the theoretical scientist to the applied engineer. 
This continuum is linked to the ontological views of the informants. At the theoretical end of the continuum, the concept is best represented in text, as there is only one reality, the abstract scientific concept. Thus, the theoretical scientist is a positivist with a tacit reality that must be made texturally explicit. While at the applied engineering end of the continuum, there are multiple realities and these realities can be represented graphically. The applied engineer is a constructivist who is comfortable with a graphical representation of their reality. Figure 11 shows the relationship of reality and textural and graphical representation of that reality. The proposal team faces the challenge of producing a proposal with an appropriate balance of text and graphics to effectively convey the author's concept. Thus, the proposal team must be mindful of the varying views of reality of the applied engineer and the theoretical scientist. One theoretical scientist stated they found little value in graphics, as their concepts are abstract. But another dealing with corrosion work explained,

"So you have to listen to the experienced voice of a graphic artist to tell you what you cannot and can do. And that really helps because sometimes you have this concept, and they look at you like, I can't do that. But I can do something else for you and maybe this will get the word, you know, the picture across for you. And that helps because sometimes you are grasping at straws with these proposals because if you have never done this before, 


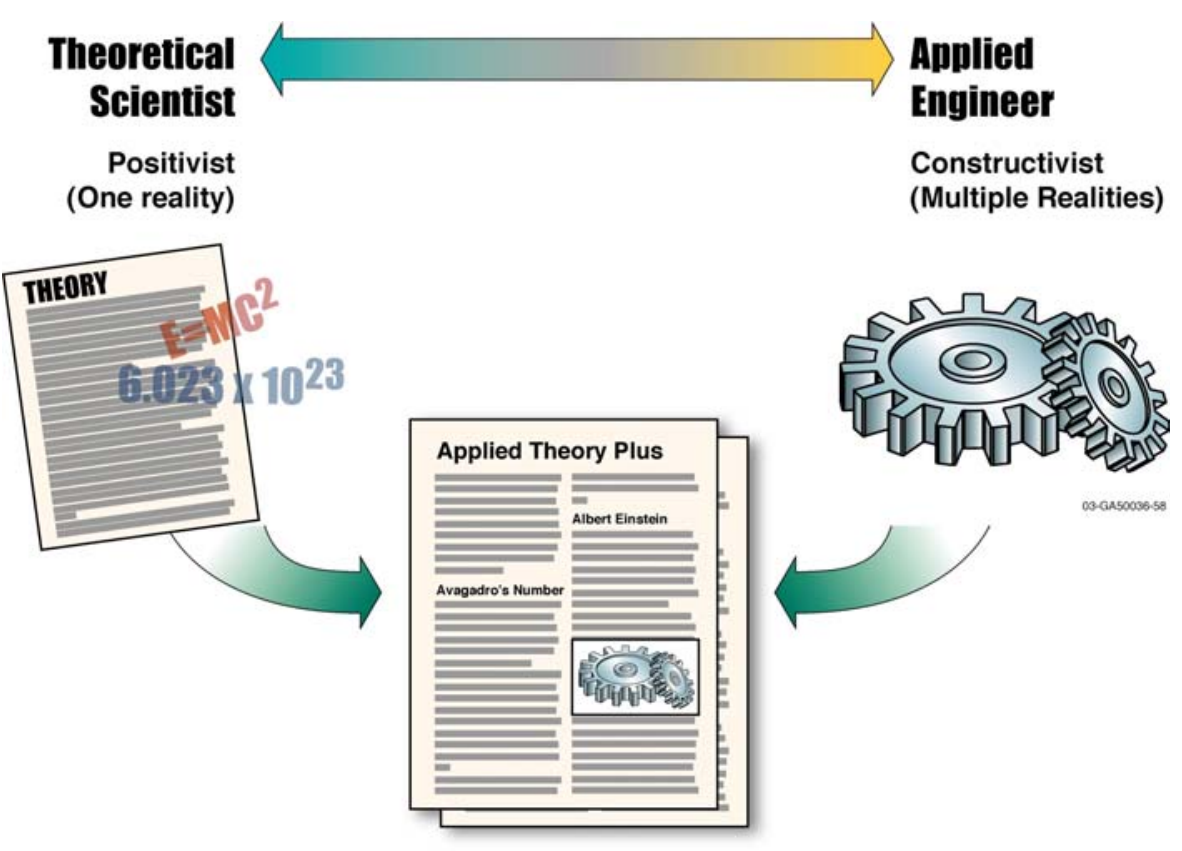

Figure 11. Textural and graphical viewpoint on the ontological continuum positioned in a science and engineering culture.

in graphics, how do you come up with something that shows you what it is that you are doing, in a nice, succinct, pretty way. And that is what customers want. They need some variety and I know, I like looking at pictures."

\section{$\underline{\text { Summary }}$}

The scientist, engineer, and manager data make the proposal processes explicit and in so doing reveal what is tacit knowledge to the proposal team. To value the explicit knowledge contribution of the heterogeneous informants, the reflective coding matrix developed using homogeneous informant data is modified and enhanced. The concept of 
the ontological continuum reveals the adaptability of the proposal team to give structure, form, and unity to both theoretical and applied concepts.

Both homogenous and heterogeneous informants are actively engaged in the human ecology of the proposal process-owning, visioning, reviewing, producing, and contributing. The heterogeneous informants added the processes of reviewing and producing to the attributes of the proposal ecology.

The presence of a proposal community of practice engaging in core and boundary practices emerges. Based on interviews with the heterogeneous informants, I received confirming data that the proposal practice is viewed as community of practice. One of the heterogeneous informants explained that they now understand more completely the proposal practice. When they worked with the service it was an emerging process with "training wheels," but some members of the proposal community of practice fully understand both the science and the proposal aspects of the practice. These proposal team members were establishing the core practices for all team members. This is truly confirmation of the existence of a proposal process community of practice that is evolving, flourishing, and adapting. 


\section{Chapter 6}

\section{Emergence of Substantive Theory}

The reflective coding matrix shown in Table 5 integrates homogeneous and heterogeneous informant data, debrief data, and the researcher's journal entries and memos to establish five evolving processes of the proposal community of practice. The processes of owning, visioning, reviewing, producing, and contributing are intimately linked to a strategic business process and strategic learning. But is the proposal team an interdependent, organizational community or simply a collection of individuals working independently on the same task? The answer to this question focuses the grounded theory interpretation to determine if the proposal team is a work group, a team with independent disciplines completing a task, or a community of practice, an interdependent team pursuing a shared enterprise (Boyatzis, 1998; Forsyth, 1999; Wenger, 1998). The second focus is to determine if the team is an ecosystem in a human ecology. To be an ecosystem there must be interdependence among members, evidence of group cohesion, and evidence that group characteristics move beyond the individual characteristics of the members (Capezio, 1996; Odum, 1959; Wolff, 1991). To start the interpretation process, one needs to reflect on the strategic learning processes necessary for the evolution of a proposal community of practice.

\section{$\underline{\text { Researcher Reflections }}$}

The power of grounded theory is the fit of data as theory emerges. The tacit is made explicit enabling the theory to be actionable. Traditional organizational learning literature provides sweeping statements of how to improve processes divorced from context, the organizational culture of the process, and fails to explicitly state the strategic benefit of the processes and resulting product. Only Argyris (1993), Glaser (1964), 
McCaslin (1996), Robey, Khoo, and Powers (2000), and Wenger (1998) provide explicit detailed accounts of the content and context of their research. Additionally, organizational learning tends to focus on increases in production of product or sales goals rather than the highly academic pursuit of proposal writing. As I develop the substantive theory of a proposal community of practice, there are instances when the voice of the informants are not echoed in the extant literature because of the unique content and context of our practice. There is a true need in the qualitative research community to make explicit the processes and tools used to discover theory so others can enhance their practice (Anfara, Brown, \& Mangione, 2002; Carlson \& McCaslin, in press).

The resulting substantive theory on proposal community of practice is grounded in the informant data and provides insight into a research approach that looks at the culture of the research problem as well as the purpose of the research.

\section{$\underline{\text { Interpretation }}$}

To establish if the proposal team is a community of practice, I used Wenger's (1998) indicators for the presence of a community of practice.

1. Sustained mutual relationships-harmonious or conflictual

2. Shared ways of engaging in doing things together

3. The rapid flow of information and propagation of innovation

4. Absence of introductory preambles, as if conversations and interactions were merely the continuation of an ongoing process

5. Very quick setup of a problem to be discussed

6. Substantial overlap in participants' descriptions of who belongs

7. Knowing what others know, what they can do, and how they can contribute to an enterprise

8. Mutually defining identities 
9. The ability to assess the appropriateness of actions and products

10. Specific tools, representations, and other artifacts

11. Local lore, shared stories, inside jokes, knowing laughter

12. Jargon and shortcuts to communication as well as the ease of producing new ones

13. Certain styles recognized as displaying membership

14. A shared discourse reflecting a certain perspective on the world. (pp. 125-126)

Without exception the proposal practice meets all fourteen indicators for the evolution of a community of practice. Therefore, the proposal service is a community of practice in the setting of the research and applied engineering laboratory human ecology.

Within the proposal service community of practice, I looked for the presence of organizational learning, a human ecosystem with interdependence, cohesion, group traits, and an energy source, creative tension, core and boundary activities, and a competencycapacity-capability strategic learning approach to work. Hughes (2002) explains the sustainable competitive advantage communicators' (proposal team members) practice should add to organizational learning.

Technical communicators can add to their current value proposition by showing that their value goes beyond being cost-effective providers of information to end users. An additional role, that of an agent of organizational learning, carries additional value to the organization's competitive position. (p. 283)

\section{Organization Learning}

The team truly is a community of lifelong learners. Three adult learning perspectives-apprenticeship, developmental, and nurturing-blend to explain the team's organizational learning style. The apprenticeship perspective allows the proposal team to 
establish a group learning culture to enhance and adapt the proposal practice through active negotiation of the team's practice knowledge with the strategic goal of preparing winning proposals. Palmer (1998) talks of the value of shared practice for members "to discover one another and form communities of congruence that offer mutual support and opportunities to develop a shared vision" (p. 166). Senge (1990) provides insight into the value of a shared vision of practice.

Shared vision is vital for the learning organization because it provides the focus and energy for learning. While adaptive learning is possible without vision, generative learning occurs only when people are striving to accomplish something that matters deeply to them. In fact, the whole idea of generative learning-'expanding your ability to create'-will seem abstract and meaningless until people become excited about some vision they truly want to accomplish. (p. 206)

As part of embracing the apprenticeship learning perspective, senior team members facilitate the learning of the junior members; members blend their disciplines to achieve and enhance the proposal artifacts; the proposal practice adapts based on team knowledge gained from each proposal effort; proposal practice knowledge converges to the core of the team's proposal practice through debriefs and team collaborations; and the team prepares proposal tools to make the proposal process explicit (Hughes, 2002). The team's core values are enhanced through apprenticeship as a sustainable way to share proposal practices (Liedtka, 1999). The community's practice provides a structured process for tacit knowledge as well as tacit ignorance to become explicit through mutual support and a shared focus of proposal practice (Hughes, 2002). 
The developmental perspective of learning employs the collective knowledge of the participants to generate new knowledge. The proposal team uses their shared vision, discipline knowledge, and an organizational learning strategy to improve their practice. This perspective "emphasizes a qualitative change in learners rather than a quantitative one; learning has to do with knowing differently rather than knowing more" (Arseneau \& Rodenburg, 1998, p. 117). A potential benefit of developmental learning is a shift to an internal locus of control in the learner manifested by intrinsic motivation to make meaningful contributions (Brown, 1996).

The developmental perspective values the knowledge and abilities of the group to solve problems without the use of outside specialists and to incorporate into their core practices the knowledge learned through the action of preparing proposals (Revans, 1998; Senge, 1990). For action learning to move to the core of practice, reflective and reflexive practices must occur and result in the team deepening their understanding of their practice. Reflection is the process of turning experience into learning by making tacit knowledge explicit through dialogue (Boud, 2001; Brookfield, 1995; Dixon, 1998; Liedtka, 1999; Storck \& Hill, 2000). Reflexivity occurs when there is a conscious effort to determine what forces regulate actions, to unthink the commonplace, and remove traditional distinctions to develop a new understanding based on new information (Arseneau \& Rodenburg, 1998; Denzin, 1997; Golden, 1992; Pedler, Burgoyne, \& Boydell, 1997). The power of reflection and reflexivity is the creation of the possibility for action to vary independently from the culture and the creation of new learning capacity (Golden, 1992).

The proposal team works synergistically to dialogue about practice steps and tools that work and parts of the practice requiring change. Many of the legacy processes in 
technical publications force blind conformity, increase cost, and reduce time efficiencies so the team must be diligent to reflect and reflex beyond the status quo and to take initiative to solve problems (Bennett, Fadil, \& Greenwood, 1994; McDonough, 2000; Robey, Khoo, \& Powers, 2000). Senge (1990) describes the status quo trap by stating, "When placed in the same system, people, however different, tend to produce similar results" (p. 42). McDonough (2000) names those who promote the status quo trap as the highly non-innovative, change adverse defenders. To avoid being defenders, team members collaboratively work through problems, establish a better approach, present their suggestions to the team for critique, and assign a team member or members to actively implement the agreed upon practice step or tool enhancement (Hughes, 2002; Wenger, 1998; Senge, 1990).

Hughes (2002) explains the value of an organizational focus on the developmental approach to learning as the team "escalate[s] knowledge within an organization, thereby increasing the value of that organization's knowledge assets" (p. 280). They "promote knowledge to [other] organizational levels through artifacts such as templates and through knowledge management systems" (p. 283).

The developmental approach incorporates the knowledge of the entire team to reach resolution but depends on members to be both reflective and reflexive about their practice. The power of this team learning approach flows from the fact that the team makes informed decisions based on their knowledge rather than accepting the mandates of management. The team evolves practice always mindful that they must develop knowledge that benefits the enterprise of the laboratory, winning proposals.

As the team increases their value to the laboratory, their reflective and reflexive processes generate a creative tension adding a sense of urgency and importance in their 
developmental learning process as well as revealing areas of knowledge deficit. For example, the proposal team is using a task group to research software products to speed the production process. The selection of better production software involves searching the available literature, dialoguing with text processors, editors, graphic artists, and current software users to establish the merits of potential alternatives. The ultimate decision impacts the team practice so the members are carefully moving through the developmental learning process to ensure new software improves practice, saves time, and improves proposal quality.

Through interpretation of the data, a third learning facet of the proposal practicecaring for each other-was revealed. Thus, being a member of the practice introduces the nurturing perspective of learning. In this perspective there is a "sensitivity toward, and empathy with, learners' subjective experience of content and context" (T'Kenye, 1998, p. 163). Nurturing adds the very interpersonal aspects of sensitivity and empathy for others as a valued team member. Also, the team must feel safe and free to explore their practice-risk is acceptable to improve practice (Capezio, 1996; Jones \& Cooke, 1998; Liedtka, 1999; Pedler, Burgoyne, \& Boydell, 1997; Senge, 1990).

Allowing team members to develop a sense of imagination is another vital part of nurturing (Wenger, 1999). The ability to imagine increases both team and personal esteem and efficacy (T'Kenye, 1998). Nurturing adds a feminine component to the proposal practice-a collectivism dimension of caring in a laboratory culture that promotes individualism and justice (Hofstede, 1997; Liedtka, 1999). The collectivist approach to proposal practice provides an antidote to burnout because of the team's collaborative approach to overcoming and achieving (Liedtka, 1999). Liedtka (1999) shares the impact caring has on a community of practice when she writes, 
Such a community has a strong moral foundation of values that (a) respects each individual's unique capacity to grow and, in doing so, to contribute to the community's purpose, (b) recognizes each member's responsibility to help those within their reach to develop their abilities, (c) conveys an obligation to engage in honest dialogue with each other, and (d) includes an uncoerced agreement to subordinate short-term self-interest in return for the benefits of full participation in the life of the community. (p. 13)

Team members support each other. A team member facing a large workload can count on other team members to assist with parts of the work. The text processors can count on team members to assist in the final lay-up step even if the assistance is needed outside the normal hours of the workday. Team members are proud of the contributions they are making to the laboratory as the proposal win rate increases in part due to their collective communication skills.

The team learning perspectives of apprenticeship, developmental, and nurturing are important for the team to evolve and flourish in a traditional organizational culture. Strategic organizational learning is at the top of Maslow's hierarchy of human needs, but "our traditional organizations are designed to provide for the first three levels of Maslow's hierarchy of human needs: food, shelter, and belonging” (Senge, 1990, p. 347). Thus, the traditional organization fulfills only the owning process of the proposal practice. But for the team to learn strategically, the other four processes are essential. Maslow confirmed that high-performing teams share a sense of vision and work toward a meaningful goal (Senge, 1990). Each team member "considers work highly meaningful and challenging, works at complex tasks, uses a variety of skills, and is able to see 
complete units of work output through to completion" (Brown, 1996, p. 251). In the twenty-first century, there is a need to evaluate organizational learning and consider including addition needs, such as respect, harmony, caring, and duty to the hierarchy of human needs (Hostede, 1997).

\section{$\underline{\text { Human Ecosystem-Core and Boundary Practices }}$}

The apprenticeship, developmental, and nurturing perspectives of adult learning instill the team members with the essential attributes of an ecosystem-interdependence of members, group cohesion, reflective and reflexive approaches to building practice knowledge, and true caring for each other as part of their practice. This strategic human connectedness allows the proposal practice ecosystem to evolve, flourish, and adapt with each proposal effort by ensuring the practice improves. The team participates in a collaborative practice involving a "high degree of both interdependence and complexity" (Donoghue, Harris, \& Weitzman, 1999, p. 50). Interdependence involves the degree to which individuals and organizations need to collaborate and interact. Complexity involves the degree to which team members need to apply their judgment to interpret a variety of information (Donoghue, Harris, \& Weitzman, 1999).

But interdependence does not mean that the proposal practice is harmonious. An adaptive ecosystem faces function and dysfunction at its core as well as with its boundary practices as the status quo is challenged and forced to unfreeze (Argyris, 1993; Liedtka, 1999; Schein, 1993). There is tension within the team, as each proposal effort requires active negotiation of both new and established information to develop tools, work with authors, and produce artifacts of value. No matter how evolved the practice is, there is always tension as the tacit information, each author's concept, must be made explicit. The team uses this tension to establish a new reality for each proposal process. 
Senge (1990) calls the gap between the team's vision and the reality resulting from each proposal effort, creative tension. This tension is a source of energy for the ecosystem as each proposal effort challenges and renews the proposal team's practice, deepens their learning capacity, and recharges their perseverance and patience.

Another source of creative tension arises when the proposal team must make their producing and reviewing process knowledge explicit to scientists, engineers, and technical managers. The team sees this process knowledge as tacit as they "are often unaware of having learned to do these things, and simply find themselves doing them" (Johnson \& Pratt, 1998, p. 93). To facilitate and enhance their focus on customer needs, team members must actively dialogue with customers about the reviewing and producing processes. Editors work closely with authors and discuss restructuring or rewriting of proposal sections to increase readability and concision (Henning, 2003; McKenna, 1997; Wick, 2000). Editors apprise authors about the progress of their proposal as it moves through the text processing and printing phases. Added production checkpoints involve author approval of the draft and final lay out prior to printing or to preparation of an electronic submittal document.

Creative tension, the energy source for the community, is critical for a community of practice to negotiate shared meanings when participating in boundary activities. The team must dialogue with the author to capture the meaning of their concept and effectively communicate that concept. Hughes (2002) talks about the value of boundary practice in technical communications. "Technical communicators negotiate meaning within development communities and between those communities and user contexts, and they capture the resulting consensus as knowledge assets" (p. 278). 
Wenger (2000b) also talks about the tension created when cross-disciplinary teams collaborate.

People confront problems that are outside the realm of their competence but that force them to negotiate their own competence with the competences of others. Such projects provide a great way to sustain a creative tension between experience and competence when our participation in a project leverages and nourishes our participation in a community of practice. (p. 237)

The team has come to realize that the dynamics of the boundary activities mean some proposal efforts will be more successful than others, but each proposal effort is an opportunity to learn and improve practice. Thus, the team looks at failure, defined as an unfunded proposal, as "simply, a shortfall, evidence of the gap between vision and current reality. Failure is an opportunity for learning-about inaccurate pictures of current reality, about strategies that didn't work as expected, about the clarity of the vision" (Senge, 1990, p. 154).

The energy in the ecosystem, core and boundary linked creative tension, allows team members to take calculated risks and experiment to align their practice vision with the reality of a proposal effort (Ford, Voyer, \& Wilkinson, 2000; Howell \& Avolio, 1993). The ability to leverage risk, to adapt and improve practice is important for the vitality of the ecosystem because risk is inherent in changing the status quo. If the team does not constantly examine the status quo and learn from mistakes, then "past success can lead to complacency and failure to adapt in the face of environmental change" (Liedtka, 1999, p. 6). 
Wenger (1998) links the power of risk with imagination when he states, "Imagination requires the ability to disengage-to move back and look at our engagement through the eyes of an outsider. It requires the ability to explore, take risks, and create unlikely connections. It demands some degree of playfulness" (p. 185). The ability to play or enjoy the proposal process is unique to the proposal team. Looking at the dimensions of each of the five processes-owning, visioning, reviewing, producing, and contributing-there are two dimensions in the owning process unique to only the proposal team. Only team members verbalized the dimensions of having fun and exciting. Even though the team puts in long hours together, they enjoy their assignments, even the trying ones, when they work as a team. "When relationships grow and start to get solid, the people involved begin to enjoy each other. Just being together can turn even unpleasant tasks into positive experiences" (Maxwell, 2002, p. 112). Additionally, the dimensions of having fun and excitement point to the cohesion of the proposal team as they have a sense of belonging, enthusiasm for their practice, a willingness to sacrifice their individual desires for group goals, and a commitment to the task (Bennett, Fadil, \& Greenwood, 1994; Forysth, 1999; McDonough, 2000). In support of the power of group cohesion, team building literature indicates, "when your team can laugh and have fun along the way, you are setting up high-performance team results" (National Press Publications, 2001, p. 21).

The proposal practice has the essential attributes of an ecosysteminterdependence, cohesion, and group features unique from the individual members features, an energy source-creative tension, and a niche in the laboratory ecologyassisting others to produce winning proposals. 


\section{Competency-Capacity-Capability}

The five processes of owning, visioning, reviewing, producing, and contributing link directly with the competency-capacity-capability models "based on the premise that the focus should be placed on the way knowledge is used to build the critical capabilities a company needs in order to succeed-on the core processes and activities that enable it to compete" (Donoghue, Harris, \& Weitzman, 1999, p. 50). The team members come to the team with skills needed to perform effectively in their portion of the proposal practicetheir unique competency. Collectively the team has demonstrated a deep capacity for learning (Gelatt, 1998). Liedtka (1999) describes capacity framed in a community of practice as the

Ability to learn new sets of skills on an on-going basis has been argued by some to represent the only sustainable source of advantage for the future. Similar to this, collaboration allows organizations to converse, learn, and work more efficiently across the silos that have characterized organizational structures. The ability to redesign processes and continuously enhance their efficiency and quality from the customer's perspective represent...value creation across all products and technologies. (p. 6)

The capability of communities of practice results in team understanding of the possibilities for integrating competency and capacity to generate strategic learning and to gain a competitive advantage.

This possibility suggests that a hypothetical organization that reflects the qualities of a community of practice, based on an ethic of care, ought to be able to create and sustain competitive advantage in a changing 
marketplace more effectively than its competitors who rely on traditional thinking about the nature of business organizations. (Liedtka, 1999, p. 15) The proposal community of practice demonstrates capability as it continually enhances its practice based on new knowledge obtained through each proposal effort. The competency-capacity-capability facet of the community of practice, its ability to learn strategically, is further developed using a conditional matrix.

\section{Conditional Matrix}

To unite the discovery nature of the human ecosystem and strategic learning perspective of the proposal practice to the problem statement-discovering essential communication tools and processes to cultivate high functioning cross-disciplinary teams engaged in proposal preparation-I developed both a tabular and graphical conditional matrix. Strauss and Corbin (1990) and Creswell (1998) discuss the conditional matrix as an aid to organize the range of consequences related to the phenomenon of interest and to promote theory emergence. Glaser (1992) explains that saturation achieved through constant comparison is essential prior to developing the conditional matrix.

We look for patterns so that a pattern of many similar incidents can be given a conceptual name as a category, and dissimilar incidents can be given a conceptual name as a property of a category, and the compared incidents can be seen as interchangeable indices for the same concept. And when we get many interchangeable incidents we get saturation. (p. 40) Using the interview, debrief, and researcher journal data, the five processes of owning, visioning, reviewing, producing, and contributing saturated as heterogeneous and homogeneous informants voices provided richness to the five processes but added no additional dimensions. 
The conditional matrix then follows saturation and builds higher-level concepts from which theory emerges.

At the point where theoretical saturation began to occur (the point in time when no new categories or properties are observed in the data) uniformities in the set of categories were reformulated into a smaller set of higher level concepts. (Partridge, 1996, p. 81)

For the balance of this chapter, interpretation and theory emergence focus on the substantive theory relating to the ability of the proposal community of practice to evolve, flourish, and adapt to the strategic advantage of the laboratory. Because constant comparison of proposal practice data achieved conceptual saturation, "a dense, rich substantive theory" (Glaser, 2001, p. 66) emerges. The substantive theory values the core and boundary practices of the proposal community of practice as discovered in the informants' culturally linked data. Development of the substantive theory moves beyond studying informant data to "studying the [basic social] process, and proceeds to generate a substantive theory of it by constant comparisons of incidents within different comparative groups in the same substantive class" (Glaser, 1978, p. 107).

Table 6 shows the conditional matrix central concept, attributes, and actionable goals. The central concept captures the ability of the proposal community of practice to evolve, flourish, and adapt to the strategic advantage of the laboratory. The essential 
Table 6

Partial conditional matrix for proposal community of practice

\begin{tabular}{|c|c|c|c|c|c|}
\hline \multicolumn{6}{|c|}{ Community of Practice } \\
\hline & \multicolumn{5}{|c|}{ INTERDEPENDENTLY } \\
\hline $\begin{array}{l}\text { Actionable } \\
\text { Goals }\end{array}$ & Proactively participating in the practice & Creating the possibilities & $\begin{array}{l}\text { Evaluating } \\
\text { and } \\
\text { enhancing } \\
\text { the potential }\end{array}$ & $\begin{array}{l}\text { Producing a } \\
\text { valued } \\
\text { artifact }\end{array}$ & $\begin{array}{l}\text { Confirming } \\
\text { strategic } \\
\text { value }\end{array}$ \\
\hline
\end{tabular}


attributes of owning, visioning, reviewing, producing, and contributing flow from the reflective coding matrix processes. But for a robust substantive theory to explain the dynamics of the social processes of the proposal community of practice, each attribute must result in an actionable goal (Argyris, 1993; Golden, 1992). The attributes and actionable goals follow:

Owning-actively participating in the proposal practice

Visioning-creating the possibilities of the proposal practice

Reviewing-evaluating and enhancing the potential of the proposal practice

Producing-producing a valued artifact through the proposal practice

Contributing-confirming the strategic value of the proposal process

\section{Competency and Owning}

In the strategic learning and organizational learning literature, much is discussed about competency. Forty-four books or articles reviewed for this manuscript discuss competency with the most in-depth insight provided by Argyris (1993), Cohen and Levinthal (1990), Cunningham (1994), Kaplan and Norton (1993), Pedler, Burgoyne, and Boydell (1997), and Wenger (2000b). Generally, the discussion about the tacit concept of competency looks at what explicit discipline knowledge or skills a person possesses. For example, a degreed knowledge in an academic discipline such as chemical engineering or a demonstrated skill such as welding is what usually comes to mind when an employer looks at adding an employee to a work group. Cunningham (1994) captures the tacit nature of competency when he states, the "subject of competence, competences, competency and competencies. This field has become complex and confusing, including the problem of which of the four labels to use. However, most people seem to talk of 
'competences' and produce lists” (p. 86). But informant data reveal competency in the proposal community of practice is much more than academics and skills.

The proposal community of practice has made explicit those competencies that are necessary for owning the process. The author brings the concept, the editor provides structure, the artist gives form, and the text processor adds unity. Each member needs to possess discipline knowledge, but in addition each member must have interpersonal competencies to be able to work on a team, engage in challenging learning, be flexible, be willing to do extra, find the work personally satisfying, enjoy the excitement of meeting deadlines, and have fun. Senge (1990) talks of the attribute of owning as personal mastery. That mastery is "the discipline of continually clarifying and deepening our personal vision, of focusing our energies, of developing patience, and of seeing reality objectively. As such, it is an essential cornerstone of the learning organization-the learning organization's spiritual foundation" (p. 7).

Additionally, personal mastery comes from being a lifelong learner with an intrinsic motivation to live life from a creative vantage point. Wenger (1998) looks at owning as the process of belonging to a community of practice. Belonging has three distinct modes-

1. engagement-active involvement in mutual processes of negotiation of meaning

2. imagination-creating images of the world and seeing connections through time and space by extrapolating from our own experience

3. alignment-coordinating our energy and activities in order to fit within broader structures and contribute to broader enterprises. (pp. 173-174) 
Thus, the proposal team's attribute of owning aligns with the community of practice attribute of belonging and the organizational attribute of personal mastery. The actionable goal of owning is actively participating in the proposal community of practice.

\section{Visioning, Reviewing, and Producing - The Capacity Potentiator}

Visioning, reviewing, and producing are interconnected steps and are the engine of the norming and performing process in Tuckman's group development model shown in Figure 12 (Forsyth, 1999). The proposal practice evolves its practice with each proposal effort. The team requires less time to norm and perform as the team's practice evolves, adapts, and builds capacity with each proposal effort. The initial storming process (shown in Figure 12) is now replaced with brainstorming before the initial meeting with authors. Brainstorming involves both reflective and reflexive processes to create tools and enhance practice aligned with the proposal call to realize time and cost

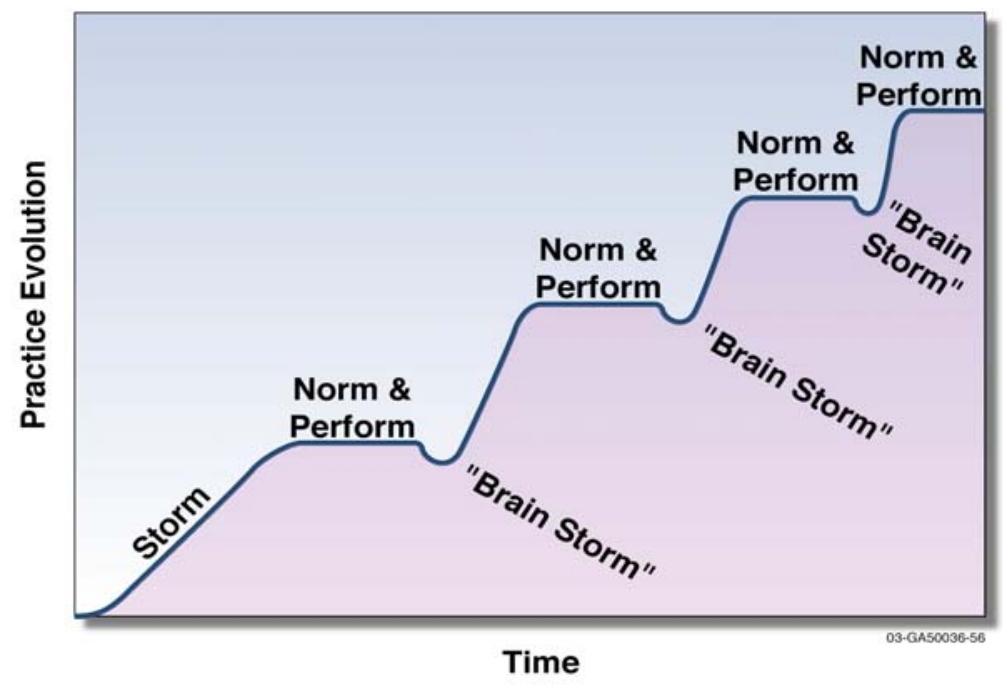

Figure 12. Proposal practice group dynamics. Note, there is no adjoining stage. 
efficiencies while producing a quality proposal document. The reason the team can achieve these levels of efficiency is because the practice process steps are explicit and actionable. Feedback and lessons learned continually amplify the process.

At the conclusion of a unique proposal activity, the debrief is part of the reviewing and visioning process rather than adjourning as explained in Tuckman's model (Forsyth, 1999). The proposal practice has no adjourning step, as the flow of work into the practice is constant. The team supports multiple efforts to bring new research and federal field office funds to the laboratory. An editor who left the proposal team for a different position within the laboratory shared in their interview that they had incorporated proposal practices into the writing processes in their new position. Evidence that group knowledge endures because the knowledge is intrinsic to each member (Drucker, 1999; Hughes, 2002).

The visioning, reviewing, and producing processes are the potentiators of the proposal practice and result in absorptive capacity creation (Johnson-Holloway, 2001; McCaslin \& Scott, 2003). Another term for the concept of potentiator is provided by McDonough (2000) when he refers to the term "reciprocal process" (p. 222) in the context of cross-functional teams. These interconnected potentiators, or reciprocal processes, simulate learning and build the capacity of the community of practice to realize the actionable goals of relating possibilities, evaluating and enhancing potentials, and producing artifacts. The cumulative effect of the three processes in combination is far greater than the effect of each process separately; thus, the cumulative impact of connecting these processes results in an exponential benefit rather than a linear, summative benefit (Cohen \& Levinthal, 1990). The potentiator builds the team's strategic learning capacity and is critical to the future of the laboratory's enterprise. Developing 
the learning capacity provides the organization with a sustainable future (Pedler \& Aspinall, 1998; Thornton, Mattocks, \& Thornton, 2000).

Dialogue is a critical part of building capacity in a community of practice because it provides an opportunity for members to share ideas, listen to each other, and value others perspectives. Dialogue provides the team with the capacity to constructively use conflict to enhance practice and create a shared vision (Liedtka, 1999; Dixon, 2000; Senge, 1990). Dialogue also creates a passion for learning. Senge (1990) relates learning and capacity when he states,

Real learning gets to the heart of what it means to be human. Through learning we re-create ourselves. Through learning we become able to do something we never were able to do. Through learning we reperceive the world and our relationship to it. Through learning we extend our capacity to create, to be part of the generative process of life. There is within each of us a deep hunger for this type of learning. (p. 14)

\section{Contributing and Capability}

The final process, contributing, is the valuing of the community's artifacts, proposals directly linked to the strategic future of the laboratory. The quantitative measure of the contribution is the new funds received by the laboratory due, in part, to the proposal practice. The quantification of contributing only provides a portion of the value of capabilities-a holistic concept that builds from competency and capacity and allows an organization to recognize the value of new knowledge and embrace change.

The proposal team works beyond the bounds of the traditional organization lines and has developed a strong customer base independent of the technical publications organization. The team supports a wide range of critical funding documents because the 
team is trusted to execute on complex, funding documents. The team's capability to thrive on change and to be successful by moving its practice outside organizational constraints enables the team to see the possibilities not the policies. This does not mean that writing standards are ignored, but a graded approach is applied to their implementation based on the type of proposal and the funding audience (Robey, Khoo, \& Powers, 2000). Senge (1990) discusses this facet of capability.

While traditional organizations require management systems that control people's behavior, learning organizations invest in improving the quality of thinking, the capacity for reflection and team learning, and the ability to develop shared visions and shared understandings of complex business issues. It is these capabilities that will allow learning organizations to be both more locally controlled and more well coordinated than their hierarchical predecessors. (p. 289)

Capabilities are the shared core practices of the proposal community that develop over time as a result of mutual engagement in the practice. Shared practices evolve from "routines, lessons learned, sensibilities, artifacts, standards, tools, stories, vocabulary, styles, etc." (Wenger, 2000a, p. 4). These shared practices are the power of the proposal community of practice as members have a scaffolding for new learning-the proposal process-that enables them "to make sense of new situations and create new knowledge" (Wenger, 2000a, p. 4). The new knowledge focuses on anticipating and responding to customer needs, analyzing ways the proposal practice can further contribute to the future of the company, and actively soliciting customer feedback to improve the practice.

But the deeper contribution of the proposal community of practice is the dynamic nature of the process. The team linked competency-capacity-capability learning approach 
uncovers opportunities to improve their practice, not because management directs the community of practice to function in that way but because the team has developed a systems approach to their practice. This systems thinking approach to work requires an understanding of how different cultural disciplines interrelate because the approach focuses on the whole.

Systems thinking finds its greatest benefits in helping us distinguish highfrom low-leverage changes in highly complex situations. In effect, the art of systems thinking lies in seeing through complexity to the underlying structures generating change. Systems thinking does not mean ignoring complexity. Rather, it means organizing complexity into a coherent story that illuminates the causes of problems and how they can be remedied in enduring ways. (Senge, 1990, p. 128)

But systems thinking can not occur until the community develops shared visions-a commitment to the future, mental models-reflective and reflexive practices to discover practice shortcomings, team learning-learning as a group and moving beyond the perspectives of any one member, and personal mastery-the motivation to continually learn how practice impacts the organization (Senge, 1990). The proposal team community of practice has capabilities in all these aspects of strategic learning.

\section{$\underline{\text { Substantive Theory }}$}

The dynamic of combining ethnography and grounded theory results in the emergence of a substantive theory grounded in the proposal practice culture. The addition of ethnography and meta-inquiry deepens the value of the research as "both the explicit and tacit knowledge familiar to most experienced members of a culture" (Spradley, 1979, 
p. 156) is discovered. Glaser (1978) links the value of ethnography to the development of substantive grounded theory, when he explains,

To generate grounded substantive theory, we need many facts for the necessary comparative analysis; ethnographic studies and direct data collection are required. Ethnographic studies, substantive theories, and direct data collection are all, in turn, necessary for building up by comparative analysis to formal theory. (pp. 147-148)

The data from homogeneous and heterogeneous informants' interviews, debrief minutes, and researcher journal entries and memos reveal the research problem of discovering essential communication tools and processes to cultivate high functioning crossdisciplinary teams engaged in proposal preparation.

The proposal practice community must be composed of interdependent, crossdisciplinary technical communicators encompassing the discipline competencies of editing, graphics arts, text processing, and production coordination. The team members must actively participate in owning and evolving the team proposal practice. The tacit proposal community of practice core practices and tools are made explicit and are valued by authors and managers; thus, confirming the grand tour question-A community of practice can evolve and be cultivated within an organization to the strategic benefit of that organization.

Once the tacit knowledge of the proposal process is made explicit, the community of practice can proactively foster strategic learning that increases the absorptive capacity of the organization. That capacity when leveraged into capability assists the organization to face change and thrive. Making tacit knowledge explicit assists the entire laboratory to 
understand the dynamics of the organizational cultures involved in a business critical proposal process.

The Proposal Team as a Strategic Community of Practice

One purpose of the research is to discover proposal tools that assist and are valued by a cross-disciplinary proposal team developing conformal, responsive proposals on a time-constrained schedule. A community of practice must produce artifacts of value to others and benefit the laboratory enterprise. For the proposal practice the valued artifacts are winning proposals. The team's essential tools for producing those artifacts include the template, guidance, author/editor checklist, technical reviewer checklist, and the timeline. The tools are valued by both the team and the authors as necessary to produce responsive, conformal proposals. These tools coupled with the proposal process provide a framework for all proposal activities. This framework allows the team to actively participate in the process while looking at the possibilities and the potentials for practice improvement and allows the team to produce artifacts of value. Thus, with each proposal the tools of the practice organize and make explicit knowledge needed to enhance and deepen absorptive capacity. This absorptive capacity is needed to appreciate the value of new knowledge and value the dynamics of change in the proposal process. Confirmation of the team's capability to holistically view their practice and think strategically results in an increased proposal win rate.

Table 6, showing the initial conditional matrix, provides a textual model of the proposal community of practice conditional matrix. Figure 13 provides a graphical representation of the same matrix. The conditional matrix provides insight into the higher-order concept-the ability of the proposal community of practice to evolve, 


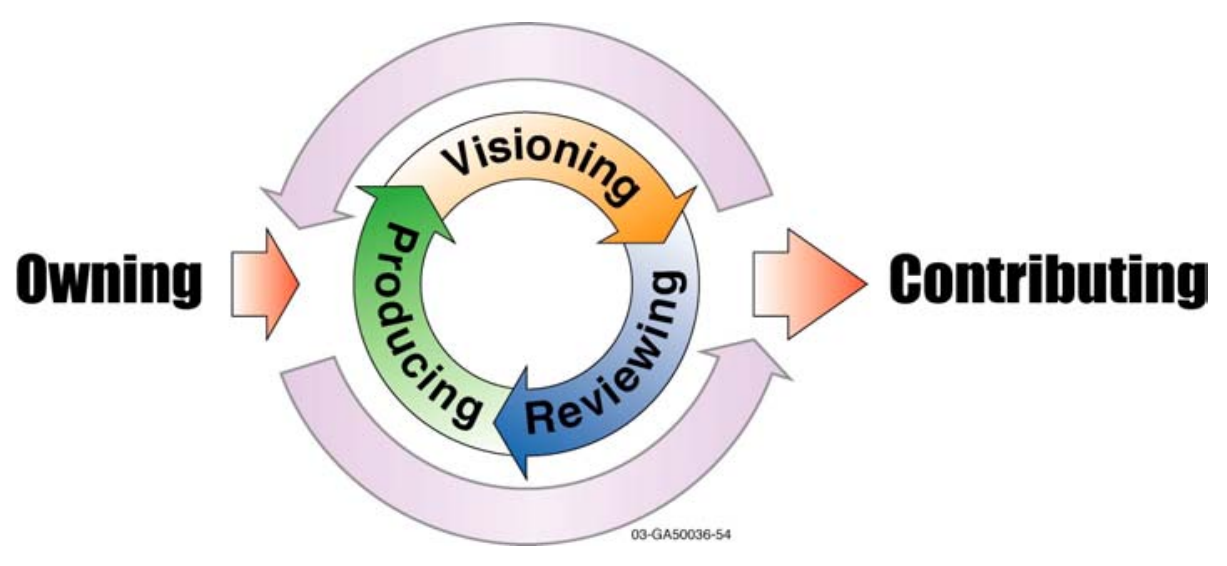

Figure 13. Proposal community of practice graphical conditional matrix.

flourish, and adapt to the strategic advantage of the laboratory. McDonough (2000), using quantitative methods, provides a model for new product development teams that incorporates the ideas of stage setters, enablers, team behavior, and performance to show team interactions. His model lacks the interconnections shown in Figure 13, but does speak to the importance of ownership, cooperation, and commitment framed in the context of work. Also McDonough (2000) affirms the efficacy of using grounded theory to discover the interconnections in organizational processes when he states, It would be appropriate to use a qualitative research approach, such as grounded theory, to generate a more detailed and in-depth understanding of the moderating effects of contextual factors, including, for example, industry and size and other elements in the model. Such research might include in-depth interviews with new product teams, as well as with individuals at multiple levels within the organization. (p. 234) 
Senge (1990) talks about the attributes of a great team as "a group of people who functioned together in an extraordinary way-who trusted one another, who complemented each others' strengths and compensated for each others' limitations, who had common goals that were larger than individual goals, and who produced extraordinary results" (p. 4). Strategic learning scaffolded by owning, visioning, reviewing, producing, and contributing in a proposal community of practice results in a great team.

The input to the proposal process is owning and the output is contributing to the funding future of the laboratory. Owning and contributing are linked as the team views completed, quality proposals as the output of their practice (Brown, 1996). Between owning and contributing, the interconnected processes of visioning, reviewing, and producing provide the potentiator of the proposal practice. The potentiator enables the power of connected visioning, reviewing, and producing to amplify practice capacity far more than any single process.

In the proposal practice-

Competency explicitly defines the attributes a proposal team member must possess to have a sense of owning. Each member must like working on a team, have good skills, find the work personally satisfying, enjoy challenging learning situations, be flexible, be willing to do extra, and enjoy having fun.

Capacity building involves actively controlling the proposal process, selling the proposal idea, creating proposal tools, valuing the proposal tools, allowing authors to focus, working with management, enhancing and reviewing the practice through lessons learned, dealing with delays in approvals, looking for scheduling opportunities, creatively dealing with 
production issues, valuing the contributions of peripheral contributors, and ensuring the reviewing process adds value.

Capability looks at the whole process by focusing on the customer, validating the feelings of customers, and creating a long-term positive impact on the company's funding future.

Figure 14 aligns competency, capacity, and capability to the proposal community of practice conditional matrix making these concepts explicit for the proposal process. Linking competency-capacity-capability with the explicit dimensions of the processes of owning, visioning, reviewing, producing, and contributing provides the potential for 


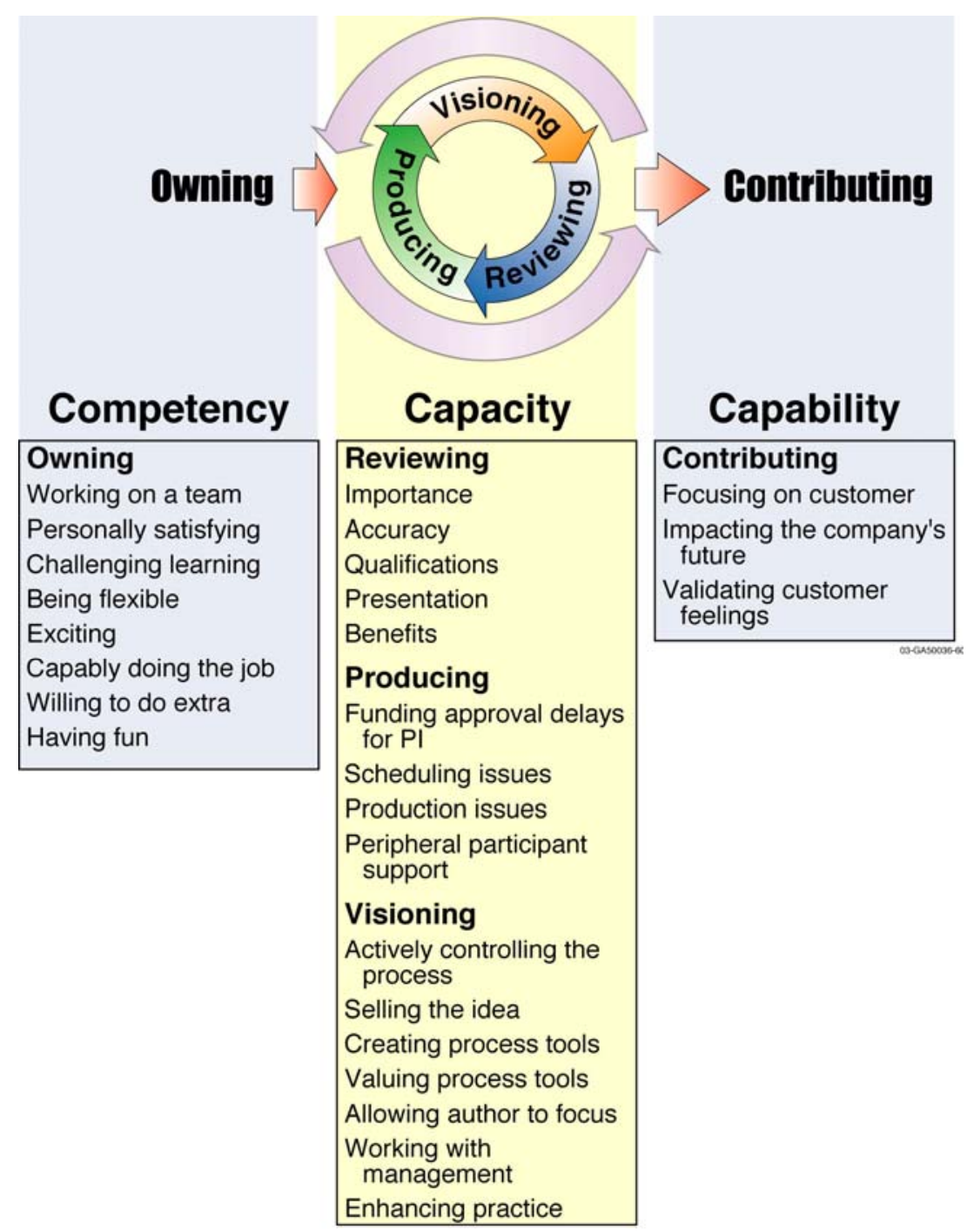

Figure 14. Explicit dimensions of competency, capacity, and capability in the proposal community of practice.

uncovering and addressing organizational learning problems. To better understand the power of the conceptual matrix to diagnose strategic organizational learning disabilities consider what happens if a process is missing or overly developed. Some simple examples are offered to make each organizational learning disability more explicit.

No owning: Members are assigned to a large proposal team to prepare a multimillion-dollar proposal. The team capably uses their skills, completes the task, and 
disbands without a sense of personal ownership of the process or product (Kent-Drury, 2000). Another manifestation of no owning is skilled incompetence, when employees actively work to keep themselves from learning (Senge, 1990).

Excessive owning: Members of a document control group develop rules and rituals that add no value to the company other than ensuring the group has work. Another term for excessive owning is stove piping or silos. Excessive owning limits or stops the flow of knowledge. (Armbecht, Chapas, Chappelow, Farris, \& et al., 2001; Senge, 1990; Wenger, 1998).

No visioning: Members work in a reactive mode, simply trying anything in an attempt to fix a problem caused by that elusive employee, the other guy (Senge, 1990) or aversion to change can result in a passion for the status quo (Woren, Ruddle, \& Moore, 1999).

Excessive visioning: This is the classic paralysis by analysis. The group that constantly plans how to do work but never executes or develops action learning (Revans, 1998).

No reviewing: Documents with poor science logic and embarrassing errors like typos, misspellings, and subject-verb disagreement are sent to a funding agency (Henning, 2003).

Excessive reviewing: The group develops a negative approach to doing work, constantly finding fault in the work of others and not appreciating the positive points (Carter-Scott, 2000). Thus, the group focuses on what is not rather than on the potential of what is.

No producing: The group that frequently misses critical deadlines and negatively impacts the organization image and the company's profitability (Dwyer, 1998). 
Excessive producing: A group that sends a constant barrage of notes, letters, procedures, standards, guidelines, and emails of marginal value to the entire organization (Benson, 1998).

No contributing: Group output does not advance the future of the company while costing the company money. Or a researcher who does not publish, propose, or work collaboratively with others (Glaser, 1964).

Excessive contributing: In the proposal community of practice this is the desired outcome balanced with reflective and reflexive practice to avoid freezing of the practice (Argyris, 1993; Schein, 1993)!

Because the proposal community core practices, content, and context are explicit, the team can more effectively collaborate with a wide variety of author disciplines (Lawrence-Lightfoot \& Davis, 1997). Figure 15 shows how the proposal community of practice must move along the ontological continuum to give structure, form, and unity to each author's concept. For a proposal community of practice to add value to the organization and to develop strategic business knowledge, three essential factors are necessary.

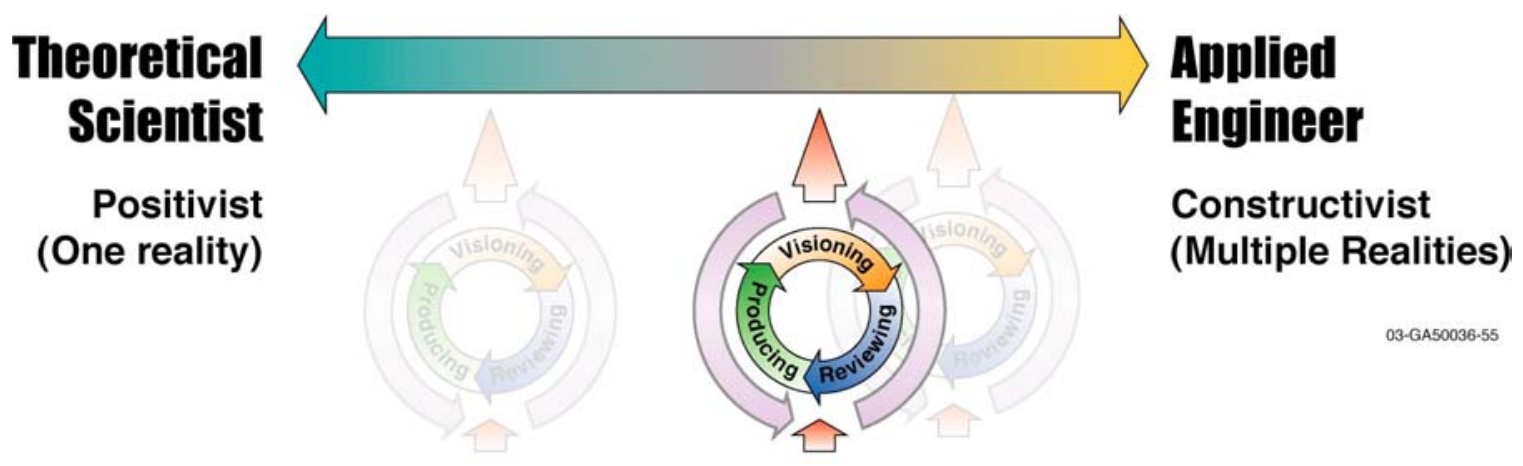

Figure 15. Proposal practice adapts and evolves to move along the ontological continuum. 
- $\quad$ The goal of the enterprise must be understandable, achievable, and interrelated to other strategic business processes; thus, the proposal team must dialogue with managers, scientists, and engineers to assist in developing winning proposals that bring new funds to the laboratory.

- The tacit cultural dimensions of community of practice-owning, visioning, reviewing, producing, and contributing-must be made explicit for knowledge products of value to result; thus, the proposal team must interdependently build their core practices through reflection and reflexivity to produce proposal tools and processes to use when working collaboratively with other communities of practice.

- $\quad$ Strategic learning must make explicit the community of practice dimensions of competency-capacity-capability for the benefit of the organizational enterprise; thus, the proposal community of practice must evolve, adapt, flourish, and take risks with the constant goal of creating new strategic practice knowledge that enables the generation of conformal, responsive proposal documents.

Table 7 posits the substantive theory in the conditional matrix. But for the substantive theory to be of strategic value it must be put into action.

\section{Actionable Substantive Theory}

Making the substantive theory actionable is ongoing with the proposal community of practice. The team actively engages in proposal or critical funding efforts. Argyris (1993) defines an actionable theory as one that,

Can be used to diagnose and understand individual, group, intergroup, and organizational behavior. Such a theory tells the person or group that uses it how to act effectively, how to design and implement actions in such a way that the actions achieve the intended consequences, they achieve these 
consequences persistently, and they do not reduce the actor's present level of effectiveness. (p. 249)

Ten actionable strategies flow from the substantive theory aligned with the proposal community of practice.

Make All Knowledge Explicit Through Dialogue: Dialogue among team members is vital to make all knowledge explicit. Editors, graphic artists, and text processors have been co-located to promote team dialogue. The co-location of the team has resulted in greater team interdependence and cohesion. Also, co-location allows team members to be aware of the workloads of other members and to move work between team members so all tasks are accomplished (Robey, Khoo, \& Powers, 2000). 
Table 7

Full conditional matrix for proposal community of practice

\begin{tabular}{|c|c|c|c|c|c|}
\hline \multicolumn{6}{|c|}{$\underline{\text { Community of Practice }}$} \\
\hline \multirow{2}{*}{$\begin{array}{l}\text { Central } \\
\text { Concept }\end{array}$} & \multicolumn{5}{|c|}{ The ability of the proposal community of practice to evolve, flourish, and adapt to the strategic advantage of the laboratory. } \\
\hline & \multicolumn{5}{|c|}{ INTERDEPENDENTLY } \\
\hline Attributes & Owning & Visioning & Reviewing & Producing & Contributing \\
\hline $\begin{array}{l}\text { Actionable } \\
\text { Goals }\end{array}$ & $\begin{array}{l}\text { Proactively } \\
\text { participating in } \\
\text { the practice }\end{array}$ & Creating the possibilities & $\begin{array}{l}\text { Evaluating and } \\
\text { enhancing the } \\
\text { potential }\end{array}$ & $\begin{array}{l}\text { Producing a valued } \\
\text { artifact }\end{array}$ & $\begin{array}{l}\text { Confirming strategic } \\
\text { value }\end{array}$ \\
\hline
\end{tabular}




\begin{tabular}{|l|l|}
\hline Tubstantive & $\begin{array}{l}\text { A business strategic community of practice can evolve and be cultivated within an organization to the benefit of that } \\
\text { organization. }\end{array}$ \\
- The goal of the enterprise must be understandable, achievable, and interrelated to other strategic business \\
processes; thus, the proposal team must dialogue with managers, scientists, and engineers to assist in developing \\
winning proposals that bring new funds to the laboratory. \\
- The tacit cultural dimensions of community of practice- owning, visioning, reviewing, producing, and contributing- \\
must be made explicit for knowledge products of value to result; thus, the proposal team must interdependently \\
build their core practices through reflection and reflexivity to produce proposal tools and processes to use when \\
working collaboratively with other community of practice. \\
- Strategic learning must make explicit the community of practice dimensions of competency-capacity-capability for the benefit \\
of the organizational enterprise; thus, the proposal community of practice must evolve, adapt, flourish, and take risks with the \\
constant goal of creating new strategic practice knowledge that enables the generation of conformal, responsive proposal \\
documents.
\end{tabular}


Establish Processes to Enculturate New Members: Each of the senior editors works closely with their junior editor interns to assist them in understanding the editing standards and the proposal process. Each junior editor is responsible for preparing the guidance, template, checklists, and timeline for a major proposal under the mentoring of a senior editor. The process of preparing the tools makes the importance of the tools clear to the junior editor. It also provides them with an understanding of how to decode grant language so the team and the authors can understand it (Kurstedt \& Mallak; 1996). This mentor/intern relationship works well as I receive few phones calls from junior editors on routine editing or proposal process tasks.

\section{Gain an Appreciation for the Scientist and Engineer Culture: All team}

members are responsible for maintaining a close collegial relationship with their assigned proposal author to ensure that the author's tacit concept is made explicit and to proactively work any concerns or issues that occur during the proposal process. The concerns can range from a family emergency, business travel, or illness. The editor makes arrangements with the author to continue working portions of the proposal in the author's absence. The editors assist the authors by initiating literature searches at the technical library, transmitting draft proposal documents to technical reviewers, restructuring text to increase readability, and attending the technical reviewer debrief to establish ways to increase the clarity of the proposal. Although these activities may appear to be of low complexity, the editor must dialogue with the author to understand the proposal topic and field questions from the research librarian or technical reviewer when the author is unavailable.

Constantly Engage in Knowledge Creation: Debriefs are held after every proposal efforts. The team meets initially to debrief on the team's view of the proposal 
process followed by a meeting with the technical manager, authors, and technical reviewers. Disconfirming information is captured in the debrief minutes and used to improve the next proposal process. No mistakes are repeated, but the very process of creating new knowledge means risks must be taken and mistakes will be made. As soon as a team member identifies a potential problem, the team leader and lead editor for the proposal facilitate resolution of the concern.

Develop a Strategy of Shared Leadership: Because the proposal process is ongoing, there is a need for multiple team members to assume lead roles. On each large proposal effort, a lead editor is assigned to coordinate the team activities based on the established timeline. Shared leadership spreads the decision-making responsibility and allows successful completion of proposal efforts (McDonough, 2000). This leadership role rotates between the senior editors so vacations can be scheduled or personal emergencies do not impact the proposal process. The lead editor and the team leader simply allocate another resource or fill the role themselves. The team cares for each other and freely volunteers to cover work even if it means long days, nights, or weekend hours (Wolff, 1991).

Avoid Establishing the Status Quo: Because of the variability of the language of each proposal call, no process is the same, nor does the team try to make them the same. Each proposal call is carefully read and the proposal tool developer uses reflective and reflexive thought processes to develop the best tools. No matter who develops the proposal tools another team member checks the tools for completeness and understandability. Junior editors check the work of senior editors just as senior editors check the work of junior editors. New eyes looking at tools make insightful suggestions and uncover cases of tacit ignorance-language that is hard to decode. The focus is to 
package information in a way that makes it more accessible and understandable by the authors (Hughes, 2002). Thus, the team exhibits alloplasticity as they easily adapt to their practice for each call (Bennett, Fadil, \& Greenwood, 1994).

Maintain Creative Tension: With the variability of each proposal call and authors knowledgeable about the topical area of the call, the team always is faced with boundary activity and core practice tensions. The general proposal process scaffolds all team efforts so only brainstorming is required before a proposal effort. The challenge is to maintain tension at a creative level rather than at a high stress level. High stress for short periods of time is expected but should never be maintained as the team can lose creativity and passion for their practice. Problem solving also results from creative tension as the team must focus on providing customer satisfaction in the dynamics of the process (Robey, Khoo, \& Powers, 2000).

Use a Collectivist Approach-Cohesion and Interdependence: Individualists are not well suited or embraced by the team. The dimensions of owning are based in the collectivist culture. Team members need to each be individuals with opinions and insights on practice enhancements, but when they function as a team, cohesion and interdependence are vital for team success.

\section{Leverage Competency-Capacity-Capability to Develop Strategic Knowledge:}

Members work individually and collectively to understand the laboratory's key strategies. They use the opportunity of working with a wide variety of authors to develop a capacity for learning new knowledge and use the knowledge to discern team capabilities that will be needed. So far the team's capacity for appreciating new knowledge has allowed us to build needed capabilities so we can better serve customers. 
Known competencies have been extremely helpful when hiring new team members. Those team members who interview potential members are able to ask relevant open-ended questions and probe in the area of interpersonal relationships. Good skills are needed to be a proposal editor, graphic artist, or text processor but more important are the interpersonal skills. The reputation of the team depends on happy customers receiving high quality documents.

Value Learning and Laughter: All team members are involved in learning on a daily basis by the dynamic nature of the work, but the team renews once or twice a year by attending an educational class together. We have lunch together after especially demanding proposal efforts. Caring for and valuing the individuals on the team is vital as each brings a special gift to the proposal community of practice. No two gifts are the same and that is the power of the team. If two people have identical skills on a team then one is unnecessary as the cross-disciplinary team depends on a good mix of talents. Debate is critical for learning and shared understanding to occur as the team embraces change.

\section{Summary}

A rich substantive theory reveals the full dynamics of the homogeneous and heterogeneous informants involved in the proposal community of practice. The discovered substantive theory is-A business strategic community of practice can evolve and be cultivated within an organization to the benefit of that organization. Visioning, reviewing, and producing potentiate the proposal practice and unite owning and contributing. Actionable goals link the five processes of the proposal practice. For the practice to adapt and flourish all five processes must be balanced. The concepts of competency, capacity, and capabilities are explicitly listed and valued for the proposal 
practice. Ten actionable strategies for the proposal community of practice emerge from the substantive theory. For the substantive theory to decouple from the laboratory proposal practice, its transferability to an overarching basic social process must be established allowing formal theory to emerge. 


\section{Chapter 7}

\section{Implications of Meta-inquiry and Community of Practice to Formal Theory}

The research process has woven the cultural threads of the proposal team, technical managers, scientists, and engineers discovered through topic oriented-ethnography into the unity of a community of practice through grounded theory. The research and applied engineering laboratory proposal service is a community of practice with actionable goals aligned with the strategic business focus of obtaining new funds. The substantive theory connects the essential communication tools and processes for the cultivating, evolving, adapting, and flourishing of the cross-disciplinary proposal team.

An understanding of the culture under study provides insights about interactions in a human ecology. The research presents an additional step, meta-inquiry, appropriate for inclusion in all qualitative methodologies. Meta-inquiry provides the researcher with a strategy to collect, analyze, and interpret data from homogeneous informants to gain an appreciation and sensitivity for their voice. Reflection and reflexivity occur as the researcher values the initial data and gains increased knowledge of the human process under study. A culturally linked grand tour question and interview protocols emerge from meta-inquiry allowing the researcher to understand the voice of the heterogeneous informants and discover substantive theory.

Broader application of the research relates to the use of meta-inquiry as an effective addition to interview protocol in all of the qualitative traditionsphenomenology, biography, and case study-as well as ethnography and grounded theory. The cultural sensitivity achieved through meta-inquiry allows the researcher to develop culturally meaningful formal interview questions in qualitative research and also provides 
a framing for the development of quantitative assessment tools (Carlson \& McCaslin, in press; Tashakkori \& Teddlie, 1998).

Meta-inquiry and formal interview data combine to explore the evolution and adaptation of a community of practice framed in a strategic business process. This final chapter moves the concept of community of practice beyond the studied research and applied engineering laboratory by providing external validity of the practice, by considering community adjourning implications, by establishing the possibilities for strategic learning situated in a community of practice, and by exploring the linkage of community of practice and leadership.

Implications of Meta-inquiry

To fully appreciate the impact of meta-inquiry on the development of substantive theory, consider the theoretical position developed based on the voices of only the homogeneous informants. Their practice concept involved owning, visioning, and contributing, as the processes of reviewing and producing are tacit knowledge to the team. The heterogeneous informants enhanced the concept of practice to explicitly include reviewing and producing as essential processes in the proposal practice. The power of making those processes explicit revealed the value of the interconnected processes of visioning, reviewing, and producing as the potentiator of the proposal practice.

Without a full understanding of the human ecology involved in the proposal practice, the research may have stopped with the emergence of a theoretical position and a linear view of the proposal practice. Figure 16 graphically reveals the dynamic of the addition of meta-inquiry before formal interviews to the qualitative research methodologies used in this research. Meta-inquiry combined with theoretical 


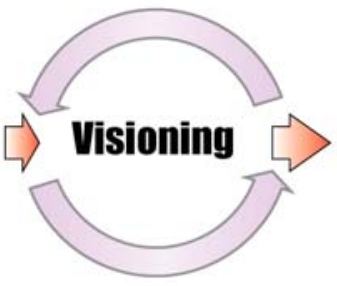

\section{Contributing}

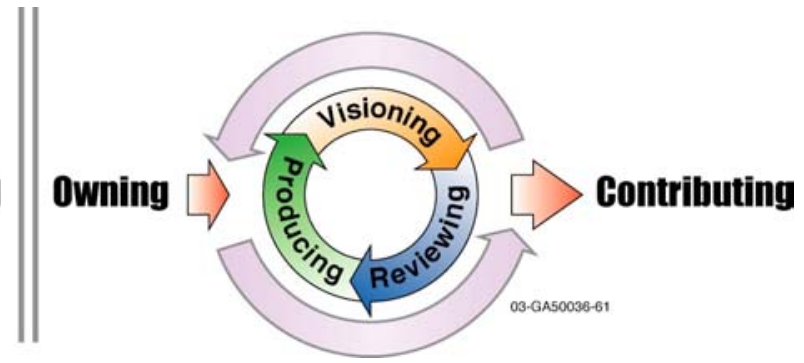

Figure 16. Meta-inquiry discovers-owning, visioning, and contributing-and enhances the researcher's awareness of the proposal ecology allowing the emergence of substantive theory including reviewing and producing.

conditioning provides a deep understanding of the human ecology being studied, the proposal service, allowing a fuller appreciation of the place in which the proposal service fits in the laboratory ecosystem. The result is a rich substantive theory that reveals the full dynamics of the homogeneous and heterogeneous informants involved in the proposal community of practice. The discovered substantive theory is-A business strategic community of practice can evolve and be cultivated within an organization to the benefit of that organization.

To move the substantive theory to a higher concept level involves moving the theory beyond the spatial and temporal limitations of the laboratory proposal practice. The higher conceptual level must move the theory to basic social processes with no bounds of space or time. Meta-inquiry, topic-oriented ethnography, and grounded theory prepare the researcher for the next phase of exploration. Glaser (1978) confirms that the trajectory to develop the substantive theory by blending the methodologies of ethnography and grounded theory leads to rich formal theory. But as one follows the 
suggested trajectory to a dense formal theory the theory becomes more general as "dedensification" (Glaser, 1978, p. 153) occurs when comparing substantive theories. Before looking across related substantive theories, interviews with corporate proposal team members provided external validation of essential competencies and capacity for proposal team members and the importance of a proposal process framing practice. This first step is important to establish the substantive theory is not place and participant bound.

Valuing the Substantive Theory - External Validity

Establishing the degree of cultural linkage of the substantive theory to the unique human ecosystem researched is a necessary first step to formal theory development. Anfara, Brown, \& Mangione (2002) provide the following insight:

Internal validity is concerned with how trustworthy the conclusions are that are drawn from the data and the match of these conclusions with reality, while external validity refers to how well conclusions can be generalized to a larger population. (p. 33)

To establish external validity, an interview with the head of a corporate proposal service and the production leader occurred. The corporate proposal team is located on the East coast and draws on an entirely different member pool than the laboratory proposal team. The corporate proposal effort is leader-focused with the technical publication staff mobilized only when a proposal effort is underway; thus, the corporate proposal team has a low level of owning by the technical publications staff (Kent-Drury, 2000). The interviews confirmed the competencies, capacity, and capabilities needed in team members for a successful corporate proposal effort. 
The corporate team leader and production leader are only tasked with multimillion-dollar proposal efforts that occur on an as-needed basis. The center captures millions of dollars on huge proposals with a win rate of nearly $100 \%$ using a process based proposal method. Before implementing the current corporate proposal method, the win rate was low with only a $25 \%$ success rate. The winning process involves a four-step approach-contract, storyboard, mock-up, and text training. From this portion of the interviews, I discovered that an explicit process is vital for a winning practice both at the laboratory and the corporate proposal center.

The corporate center has no full-time technical publications staff but there is a core group compromised of the proposal service leader, production leader, administrative support, and a proposal service manager. This is a traditional work group structure, and I could draw no conclusions about the core group's attributes as related to a community of practice as both interviews were done away from the corporate proposal ecosystem. The proposal team leader interview was done in person and the production leader's interview occurred on the phone.

With those limitations in mind, there is still great value in the interview data as they allowed me to focus on the basic social processes of the proposal practice rather than on the team members. Desirable corporate technical publications members' competencies are flexibility, cheerfulness, and skill level. The corporate staff's biggest problem is finding graphic artists and text processors with the needed proficiency as well as skills with software. Sometimes it takes several tries to find the right person as the corporate team obtains their technical support staff from the local labor pool through subcontracting for services. Another problem with the subcontracting of team members is the impact of stress at the production phase of the process. The team leader indicated that production is 
the point in the process where they see problems with burnout, as many of the subcontracted production people just cannot handle the stress. The corporate team leader indicated they have contract people who refuse to work another proposal due to the stress level. Additionally, the subcontracted members see no direct benefit from a proposal win as their services are contracted only to assist in preparing the document with no job offer potential for good performance. To keep members motivated, the corporate group gives rewards like mugs, handshakes, or T-shirts.

The needed competencies of the corporate team align with competencies of the laboratory proposal team. The problem of burnout is not experienced at the laboratory as all members are interdependent and function as a cohesive team. Members assist each other to achieve the goal of proposal completion. A community of practice is a nurturing environment with group welfare of great importance. Although we go to lunch together to celebrate after huge projects, laughter and friendship appear to be an adequate reward for a caring community of practice.

The entire corporate team co-locates to simplify the writing process as all members-authors, managers, technical publications, and the corporate core team-are in the same location during the writing effort. At the laboratory the core team is co-located but the authors and managers maintain their normal work location. That location is many times different from the building in which the laboratory proposal team is co-located. Also the corporate team has a strong senior management champion to make performers adhere to the process. Although the technical manager plays that team role in the laboratory proposal process, the technical manager is faced with many situations in which they have limited authority. An empowered technical manager leading the proposal process is a needed area for improvement in the laboratory's proposal practice. 
To assist in the development of needed strategic knowledge about a proposal call, the corporate team uses capture sheets. A portfolio manager develops the capture sheet during visits with the agency or company requesting proposals. The sheets provide the team with data on the proposal's critical winning points, funding agency sensitivities, and details on the competition's strengths and weaknesses, so the corporate proposal team can articulate the needed winning strategy. This level of political reconnaissance is lacking at the laboratory, as the proposal community of practice rarely has detailed information about the competition. These data are vital for the team to more effectively address the funding agency's true needs and to increase the laboratory's proposal win rate. Senior managers in the laboratory recognize this fact, and actions appear to be underway to correct this strategic knowledge issue.

The corporate proposal service uses a 50:50 strategy to prepare documents. The proposal team leader indicated that their goal is 50\% graphics. Additionally the proposal team leader has final say on all text and graphics. The corporate team has a firm timeline and requires all author pens must go down at the same time so the proposal center can do their job. Based on the laboratory's current proposal practice, there are opportunities to improve our practice based on the corporate approach. Concepts like receiving capture sheets for each proposal effort and allowing the proposal team adequate time to unite the document would enhance the laboratory proposal practice. Graphics have been introduced into all proposals, but a better balance of text to graphics is a goal the team strives to achieve.

From the corporate team member interviews, I discovered no new competencies, capacity dimensions, or attributes different from the substantive theory. There are areas of the corporate process that would improve the laboratory practice and can be 
incorporated into our visioning process through the dimension of enhancing and reviewing practice. I could only conclude that the capabilities of the corporate core group are acceptable based on their incredible win rate.

Based on laboratory proposal community of practice data, my interviews of the corporate proposal team members, and the extant literature on proposals, community of practice, and technical communication, there is both internal and external validity in the substantive theory. But the substantive theory, although valid, is transferable and generalizable to only a proposal community of practice without further theory development.

\section{Community of Practice Adjourning Impacts}

Capezio (1996) calls the adjourning process, disbandment, when he speaks of the morale and productivity impact of ending team activities. He states, "If the experience has been positive, team members may be upbeat about their success, thereby projecting a positive morale" (p. 48). To explore this idea, I had the opportunity to dialogue with a manager of a group that had adjourned. The adjourned community of practice surfaced in discussions with the qualitative researcher who assisted in the audit trail for the research. Cross-disciplinary scientists, engineers, and technicians had been assembled to provide environmental solutions across several laboratory sites within the federal complex. After several years of working environmental issues with much success, federal funding was redirected and the community was without an advocated goal or fund source. The manager who had directed the team explained the impact of adjourning on the team.

"In most cases, these groups of people had very proactive and productive activities going on, okay...there was that first disappointment and rejection and what did we do wrong because we were making progress. 
We were moving waste. We were doing things to help these sites. These sites were calling us and depending on us and now it's gone, gone away. Once they got over that, you know, their skill sets were still there. They knew they could still contribute and they have stood up and moved on and are doing it....The majority of folks have gone back to their technical roots, their technical areas of expertise and are helping either laboratory-based projects or either closure projects."

The adaptability of a community of practice is evident in the manager's story. Members grieved a bit but saw opportunities to leverage their skills in other ways. Thus a community of practice provides support for building practice, but the practice knowledge moves from the community to benefit other organizations. The members own their practice and their contributions move beyond the community to benefit the organization.

\section{Formal Theory Emergence}

The processes of active owning, visioning, reviewing, producing, and contributing by the community uncovered three basic social processes-interdependence, caring, and cohesion. These social processes are joined by the power of joint enterprise into a community of practice aligned with active learning. The business critical nature of the practice has the potential to provide a path to strategic learning. Cunningham (1994) states, "Strategic learning starts with strategic imperatives-the direction the organization is taking, its purpose, its vision, its mission, its strategic goals" (p. 1). Thus the formal theory to emerge from this research focuses on strategic learning in a community of practice. To link the substantive and formal theory Glaser (1978) states, The linkage between research data and formal theory, provided by substantive theory, is twofold. It occurs when a particular substantive 
theory is extended and raised to formal theory by the comparative analysis of it with other research data. The linkage occurs also when the substantive theory is comparatively analyzed with other substantive theories. The theory arrived at when comparing substantive theories is more general and more qualified. (p. 146)

\section{Extant Literature Studies of Practice}

There are several studies in the extant literature focusing on strategic group learning. But only a few provide insight into the dynamics of strategic learning linked to the community of practice concept of joint enterprise. Robey, Khoo, and Powers (2000) provide insight into the need for such an integrated formal theory in the concluding text of their study of cross-functional virtual teams. They state,

The implications developed here reflect an awareness of virtual teams as distinct communities, capable of learning their particular practices.

However, we expect that the processes of learning and communication described here would occur in some fashion in every virtual team faced with the need to coordinate across business functions. (p. 64)

Robey, Khoo, and Powers (2000) point to the need for additional research into teams involved in community of practice processes. To that end, I have combined the studies linking community of practice and the potential for strategic learning in Table 8. Study results are aligned to the discovered processes of owning, visioning, reviewing, producing, and contributing. Unless the author explicitly discusses one of the discovered processes, data are not listed on the chart. Blank table cells indicate no substantial discussion of that process by the researcher. The power of comparing substantive theories positioned in areas other than proposal development decouples the processes of owning, 
visioning, reviewing, producing, and contributing from context of proposal practice so formal theory can emerge. 
Table 8

Explicit studies related to communities of practice in an organization

\begin{tabular}{|c|c|c|c|c|c|c|}
\hline \multicolumn{7}{|c|}{$\underline{\text { Strategic Group Learning }}$} \\
\hline $\begin{array}{l}\text { Carlson, } 2003 \\
\text { Proposal Process } \\
\text { Goal = gain funds }\end{array}$ & $\begin{array}{l}\text { Actively } \\
\text { participating }\end{array}$ & $\begin{array}{l}\text { Creating the } \\
\text { possibilities }\end{array}$ & $\begin{array}{l}\text { Evaluating and } \\
\text { enhancing potential }\end{array}$ & $\begin{array}{l}\text { Producing a } \\
\text { valued artifact }\end{array}$ & $\begin{array}{l}\text { Confirming } \\
\text { strategic value }\end{array}$ & $\begin{array}{l}\text { Looks at a proposal } \\
\text { team community of } \\
\text { practice in a research } \\
\text { and applied engineering } \\
\text { laboratory }\end{array}$ \\
\hline $\begin{array}{l}\text { McCaslin, } 1996 \\
\text { Community Development } \\
\text { Process }\end{array}$ & $\begin{array}{l}\text { Purpose of } \\
\text { participation }\end{array}$ & $\begin{array}{l}\text { Purpose of the } \\
\text { process }\end{array}$ & & $\begin{array}{l}\text { Purpose of } \\
\text { possibilities }\end{array}$ & & $\begin{array}{l}\text { Focuses on rural } \\
\text { community } \\
\text { development in the } \\
\text { mid-West }\end{array}$ \\
\hline
\end{tabular}




\begin{tabular}{|c|c|c|c|c|c|c|}
\hline $\begin{array}{l}\text { Argyris, } 1993 \\
\text { Eliminate Organizational } \\
\text { Defenses } \\
\text { Goal = learning organization }\end{array}$ & $\begin{array}{l}\text { Participation by } \\
\text { senior management }\end{array}$ & $\begin{array}{l}\text { Productive } \\
\text { reasoning } \\
-\quad \text { Double loop } \\
\text { learning }\end{array}$ & $\begin{array}{l}\text { Action maps } \\
-\quad \text { Reveal component } \\
\text { causality }\end{array}$ & $\begin{array}{l}\text { Desired } \\
\text { consequence - } \\
\text { learning } \\
\text { organization }\end{array}$ & & $\begin{array}{l}\text { Focuses on senior } \\
\text { management } \\
\text { interactions }\end{array}$ \\
\hline $\begin{array}{l}\text { Glaser, } 1964 \\
\text { R\&D scientist promotion and } \\
\text { recognition process }\end{array}$ & $\begin{array}{l}\text { Participation in } \\
\text { tightly defined } \\
\text { groups } \\
-\quad \text { Mentoring }\end{array}$ & & $\begin{array}{l}\text { Formal promotion } \\
\text { process } \\
\text { Informal facility } \\
\text { assignments }\end{array}$ & $\begin{array}{l}\text { National or local } \\
\text { contributor to } \\
\text { science } \\
\text { knowledge }\end{array}$ & $\begin{array}{l}\text { National } \\
\text { recognition }\end{array}$ & $\begin{array}{l}\text { Provides insight into } \\
\text { the culture of scientists } \\
\text { in a research laboratory } \\
\text { based on survey results } \\
\text { with no interviews }\end{array}$ \\
\hline $\begin{array}{l}\text { Robey, Khoo, \& Powers, } \\
2000 \\
\text { Cross-functional virtual } \\
\text { teams } \\
\text { Goal = manage supply chain }\end{array}$ & $\begin{array}{l}\text { Participation in } \\
\text { virtual cross- } \\
\text { functional teams } \\
\text { separated by } \\
\text { distance }\end{array}$ & $\begin{array}{ll}\text { Meeting } \\
-\quad \text { Business } \\
-\quad \text { Quarterly }\end{array}$ & $\begin{array}{l}\text { Approaches to } \\
\text { increase ability to } \\
\text { communicate over } \\
\text { distance }\end{array}$ & & & $\begin{array}{l}\text { Looks at a software } \\
\text { company's integration } \\
\text { of their northern and } \\
\text { southern supply chain } \\
\text { functions }\end{array}$ \\
\hline
\end{tabular}


McCaslin (1996) explores the role of reciprocating transformational leadership in community development. Community is defined by geography in McCaslin's study, but the processes of owning, visioning, and producing are present. The interplay of human interaction within a community is explored as well as the concept of a human ecology. The focus of the research is on community purpose, a holistic concept, rather than the quantitative concept of measurable goals. McCaslin (1996) explains this sense of wholeness when he states,

A holistic portrait of leadership flows from a community that has committed itself to balance found only through measures that consider product, process, participation, and possibilities as partners. These partners are held together only when a commonality of purpose can be discovered. (p. 35)

The power of participation, potential, and possibility is explored as it reciprocates and strengthens community through leadership. Thus McCaslin (1996) explores the concept of a leader as a potentiator of community. The sense of joint enterprise is not clearly evidenced as

- $\quad$ There is no active reviewing process

- The result of producing is the production of the community development process

- The value of contributing is not linked to joint enterprise with an actionable goal. Wenger (1998) explores the facets of a community of practice and establishes the presence of a community of practice in a claims processing department. He builds a strong case for the presence of joint enterprise as he explores the three dimensions of owning-imagination, engagement, and alignment. These three dimensions are components of owning voiced by my research informants, although they verbalized 
different terms. There is also a discussion of the process used to negotiate meaning, which aligns well with the visioning process. Reification talks of the concept "of giving form to our experience by producing objects that congeal this experience into 'thingness.' In so doing we create points of focus around which the negotiation of meaning becomes organized" (Wenger, 1998, p. 58). Thus reification is the process of producing artifacts.

Wenger's work on community of practice only loosely relates the essential elements of reviewing and contributing to community. A reviewing process is discussed but appears to be an external audit function to promote error free claims, the valued contribution, of the process. As these informants work to improve their practice, the production of error free claims is linked to individual reward rather than practice enhancement or community core practices.

The dilemma with Wenger's research as it relates to strategic learning in communities of practice pivots around the members of the community studied. Unlike the proposal service which engages highly skilled members and enculturates them into the practice by using the internship component of apprenticeship, Wenger's informants come to their community with little prior knowledge and are truly apprentices. Thus Wenger provides a scaffold for confirming the proposal community of practice, but the community he studied involved skills with low-complexity. In the twenty-first century there must be more research in organizational literature on internships and less emphasis on motivating sales forces and workers in low-complexity jobs. Using a community of practice focus in strategic business groups is a central approach to the promoting strategic learning, the knowledge capital of competitive advantage (Collard, 1998; Cunningham, 1994; Forrester, 1965; Liedtka, 1999). 
Argyris (1993) explores organizational defenses as he works with a company's senior management team. The hope of the management team is evolution of a learning organization. The research provides tools, action maps, to understand the defensive and productive reasoning occurring in group interactions. Thus, Argyris provides an action science methodology to uncover barriers to knowledge development and flow. Although the senior managers work with the researcher throughout the study, it is never clear if they are actively building a sense of community cohesion and interdependence as a result of researcher interventions. The contributing process is not revealed, as the actionable goal of a learning organization is not realized. Kaplan (1999) uses Argyris' approach to discover the predictability of defensive reasoning outcomes by studying budget decisions at the same research and applied engineering laboratory as my study. Kaplan's action maps provide insight into the culture of the laboratory and clarifies the value of metainquiry, as one must understand the tacit aspects of culture and make the tacit explicit. Action maps are one tool to make knowledge flow explicit.

Glaser (1964) looks at the culture of a large medical research organization. His research is based on the analysis of survey results rather than interviews with informants, but he uncovers cultural dimensions of the medical research community of practice. Owning in the medical research community is a very personal process shared with only a small group of cohorts for the benefit of the group rather than the organization. Glaser looks at the formal promotion and recognition processes as they relate to a scientist's status in the research organization. The promotion process is part of reviewing while the recognition process with the actionable goal of nation recognition for accomplished work measures contributing. The producing process relates to adding to the body of science knowledge. 
Glaser (1964) introduces the idea that many scientists will not achieve lofty goals and recognition, but rather are organizational scientists, scientific journeymen, who make only small contributions to science knowledge. The power of Glaser's research is making the tacit aspects of the scientific culture explicit. His research provides a glimpse into understanding the need for strategic learning, cohesion, and interdependence among scientists to develop communities of practice for the competitive advantage of the laboratory. The other interesting fact about Glaser's work is that the research was completed in 1964 and still holds true at the laboratory in 2003.

The final study by Robey, Khoo, and Powers (2000) looks at virtual crossfunctional teams involved in managing the supply chain for a software company. Although the study aligns with an organizational focus on sales, the study reveals the challenges of developing a community of practice separated both by distance and cultural differences based on geography. The study looks at the approaches community members employ to work practice issues, to use creativity in approaching work practices, to solve problems without management oversight using initiative, to provide customers with needed service, and to develop effective means of communicating. The study confirms the value of face-to-face communication, dialogue, to build teams even in a software technology industry. The authors state, "Our findings clearly show the value of face-toface meetings in establishing a greater social connection among team members" (Robey, Khoo, \& Powers, 2000, p. 63). Thus, this study shows that dialogue and trust are vital for teams to function effectively.

Through reviewing extant literature the processes of owning, visioning, reviewing, producing, and contributing recurred as essential for community practice. The concept of shared vision gained through negotiating meaning is essential for community 
strategic focus. The connectedness of human processes links to the concepts of potential and possibility. Learning is important for the community to adapt to change and interrelate to one another. Vibrant communities actively engage in enculturating new members. The valuing of artifacts by those outside the community establishes the true contribution of the practice. Understanding the tacit and explicit knowledge of a culture helps the researcher to value the dynamics of a culture's core and boundary practices. Lastly the power of face-to-face dialogue to build trust and bond community members is explained.

$\underline{\text { Implications for Strategic Learning }}$

The range of places, participants, and activities explored in the studies listed in Table 8 reveals the transferability and generalizability potential of these substantive theories when viewed through the lens of strategic organizational learning and community of practice. The essential processes required for strategic learning to occur are owning, visioning, reviewing, producing, and contributing framed in a process that is necessary for the current and future competitive advantage of an organization. In addition to the strategic learning processes, Bennett, Fadil, and Greenwood (1994) identified four culturally based processes that must be present in every legitimate organizational subculture - tasks must be accomplished, people must be interdependent, change and adaptation are embraced, and new knowledge is created and integrated with existing knowledge. To these four culturally based processes one must add a shared vision of the reason for the practice and actionable goals to measure achievement of the vision (Senge, 1990).

For strategic learning to occur in a community of practice, the community must provide artifacts of value. The community's artifacts must be valued by the organization 
and recognized for their value outside the organization, in the marketplace, to provide the organization with a competitive advantage (Liedtka, 1999). Strategic learning must be linked to the current and future practice by a shared meaning and vision internalized by all community members. To achieve this shared vision requires caring, cohesion, and interdependence among team members to work through changes and adapt as well as an appreciation for the power of each member's knowledge gifts.

Knowledge must be viewed by an organization as having a higher value than tangible assets (Wick, 2000). Knowledge is valued as the core of competitive advantage and is developed through dialogue (Dixon, 1998). Management must allow communities of practice to evolve by promoting active dialogue between and across disciplines to the identify possibilities and potentials. To achieve a competitive advantage, the dialogue must be informed with full vision of the future as it relates to needed competencies, capacity, and capabilities.

Communities of practice must be allowed to take risks, resolve creative tension, and create knowledge aligned with strategic practice rather than blindly following policy. The community gets work done and generates knowledge through the intrinsic motivation of members because an explicit process understood and embraced by all members scaffolds their strategic practice (Halpren, 1966). Kurstedt and Mallak (1996) state, "Intrinsic motivation should guide behavior toward the mission of the organization" (p. 10).

A community of practice has the energy of visioning, reviewing, and producing to enable members to achieve actionable goals aligned with the organization's future. But the organization must be patient as communities of practice develop and provide opportunities for strategic knowledge to be acquired, shared, and used. Ford, Voyer, and 
Wilkinson (2000) cautions that, “As utilized knowledge improves performance and those improvements are recognized as the results of organizational learning, they increase the commitment of the organization and its individuals to organizational learning, although this response is often delayed" (p. 81).

Strategic knowledge created and valued through communities of practice has application beyond any one community practice. The process of strategic learning only evolves, adapts, and flourishes through interdependence, caring, and cohesion of individuals united in practice for the benefit of the company's future. Some work groups may exhibit interdependence, caring, and cohesion but produce no business critical products. The group is a cost center, not a community of practice, in a strategic sense. Additionally, the boundary activities between communities of practice must be viewed as the location for step changes in strategic learning. The disconfirming information at boundaries challenges all community members to reflect and reflex on their practice and ways to create new knowledge for the benefit of the organization. Once tacit practice knowledge is made explicit, strategic learning increases the absorptive capacity of all engaged in the enterprise. That capacity, when leveraged into capability, assists the organization to face change and thrive in a competitive marketplace.

The processes of owning, visioning, reviewing, producing, and contributing are essential for meaningful joint enterprise. Examining these processes provides a gauge for the health of an organization. Interconnecting these processes aligned with a business strategic goal provides an organizational approach to cultivating communities of practice.

\section{$\underline{\text { Conclusion }}$}

The development of a formal theory for practice situated in a strategic learning community provides the possibility of job satisfaction and evolutionary knowledge 
creation. Use of this theory has potential applications in communities of practice that experience high burn out rates such as an emergency room team where time constrained practice limits reflective and reflexive practice. Brief discussions with members of emergency room teams indicate that their practice does not include the process of reviewing. This research indicated a strong community of practice that scaffolds members and reviews practice reduces the chances of burnout.

Understanding the processes and balance between these processes is essential for a researcher to diagnose how to improve practice. For example in a community of scientists, one may encounter a low level on interdependence and cohesion based on this research study. Thus to enhance practice one would need to focus an intervention on team development strategies to increase owning. A strategic community of practice theory also provides a methodology to establish if a group has a shared vision and is contributing to the betterment of the enterprise.

Thus the initial formal theory developed is-A strategic, cohesive community of practice evolves and flourishes to benefit joint enterprise when the members interdependently own, vision, review, produce, and contribute by

- $\quad$ Making all knowledge explicit through dialogue

- $\quad$ Establishing processes to enculturate new members

- $\quad$ Gaining an appreciation for contributions others make

- Constantly engaging in knowledge creation through active boundary activities

- $\quad$ Challenging the status quo

- Maintaining creative tension

- Cultivating cohesion and interdependence within and between communities of practice 
- Understanding the value of and leveraging competency-capacity-capability to develop strategic knowledge

- $\quad$ Valuing the power of knowledge, caring, and laughter

- $\quad$ Developing a strategy of shared leadership.

In closing, the concept of leadership as a shared process in a community of practice is explored. Before a community forms and storms there is a need for a champion to explore the potential of shared practice, but that practice must be developed collaboratively with potential core members of the community. The proposal practice was the shared vision of the initial core members-the team leader, the production leader, and a senior editor-who worked collaboratively to articulate the concept of a proposal team to laboratory senior management. Once management sanctioned the concept, the assembled editors, graphic artists, and text processors cultivated the community's practice based on a shared vision of evolving a process that would assist in bringing new work to the laboratory by winning proposals.

Along with a shared vision in a community of practice comes shared leadership. To reveal the power of the author's research concept, the team leader cultivates relationships, the graphic artist creates form, the editor applies structure, the text processor provides unity, and the production manager coordinates the final artifact. In a community of practice Rost's (1991) concept of leadership-“an influence relationship among leaders and followers who intend real changes that reflect their mutual purposes" (p. 102)-takes on a new meaning as the role of a visioning leader and supportive follower changes throughout the process. Team members share leadership in the proposal process while making their contributions in a success-focused process. Shared leadership introduces the dynamics of multidirectional interactions, multiple leaders and followers, a 
desire for substantive and transforming change, and capacity to deal with multiple changes at once (Rost, 1991).

Rost (1991) explains, “Followers may be leaders for a while, and leaders may be followers for a while. This ability to change places with changing organizational positions give followers considerable influence and mobility" (p. 109). Whether in the role of leader or follower, team members are always aligned with the strategic, actionable goal of preparing a winning proposal to ensure the future of the laboratory.

The adult learners in the proposal community of practice are engaged in strategic metagogy, a concept of learning that moves the learner beyond the leader to make a valued contribution in collaboration with others. This knowledge process depends on the interdependence of learning for the benefit of the individual as well as the community. Thus, "this community of learning is no longer driven by transactional motives and shortterm gains, but by metagogical motives and long-term vision and commitment" (McCaslin \& Scott, 2003, p. 14). Thus, the community's knowledge made actionable through competency, capacity, and capabilities creates and guides the organization's strategic future. 


\section{REFERENCES}

Anfara, V. A., Jr., Brown, K. M., \& Mangione, T. L. (2002). Qualitative analysis on stage: Making the research process more public. Educational Researcher, 31(7), $28-38$.

Argyris, C. (1986, Winter). Reinforcing organizational defensive routines: An unintended human resources activity. Human Resource Management, 25(4), 541-555.

Argyris, C. (1993). Knowledge for action. San Francisco: Jossey-Bass.

Armbecht, F. M. R., Jr., Chapas, R. B., Chappelow, C. C., Farris, G. F., \& et al. (2001). Knowledge management in research and development. Research Technology Management, 44(4), 28-48.

Arseneau, R. \& Rodenburg, D. (1998). The developmental perspective: Cultivating ways of thinking. In D. D. Pratt (Ed.), Five perspectives on teaching in adult and higher education (pp. 105-149). Malabar, FL: Robert E. Krieger.

Beane, J. A. (2000). Curriculum Integration and the Disciplines of Knowledge. In Parkay, F. W. \& Hass, G. (Eds.), Curriculum Planning A Contemporary Approach ${\left(7^{\text {th }}\right.}^{\text {th }}$ ed., pp. 228-237). Needham, MA: Allyn and Bacon.

Becker, T. E., Billings, R. S., Eveleth, D. M., \& Gilbert, N. L. (1996). Foci and bases of employee commitment: Implications for job performance. Academy of Management Journal, 39(2), 464-482.

Bennett, R. H., III, Fadil, P. A., \& Greenwood, R. T. (1994). Cultural alignment in response to strategic organizational change: New considerations for a change framework. Journal of Managerial Issues, 6(4), 474-490.

Benson, M. (1998). Creating and maintaining organized files and records. Shawnee Mission, KS: National Press Publications. 
Blackman, H. \& Gertman, D. (2002). Socio-technical organization and cognition: A socio-organizational approach to risk based assessment and design. Manuscript in preparation.

Boud, D. (2001). Using journal writing to enhance reflective practice. New Directions for Adult and Continuing Education, 90, 9-17.

Boyatzis, R. E. (1998). Transforming qualitative information: Thematic analysis and code development. Thousand Oaks, CA: Sage.

Boyer, E. L. (2000). Connectedness through liberal education. In Parkay, F. W. \& Hass, G. (Eds.), Curriculum planning a contemporary approach $\left(7^{\text {th }}\right.$ ed., pp. 484-489). Needham, MA: Allyn and Bacon.

Brookfield, S. D. (1995). Becoming a critically reflective teacher. San Francisco: JosseyBass.

Brown, S. P. (1996). A meta-analysis and review of organizational research on job involvement. Psychological Bulletin, 120(2), 235-255.

Bycio, P., Hackett, R. D., \& Allen, J. S. (1995). Further assessments of Bass's (1985) conceptualization of transactional and transformation leadership. Journal of Applied Psychology, 80(4), 468-478.

Capezio, P. (1996). Supreme teams: How to make teams really work. Shawnee Mission, KS: National Press Publications.

Carlson, N. M. (March 2001). FY00 professional development fund allocation (INEEL/INT-2001-00045). Idaho Falls, ID: Idaho National Engineering and Environmental Laboratory.

Carlson, N. M. (March 2002). FY01 professional development program report. Retrieved March 2002, from http://home.inel.gov/pdhome/PDFY01ProgramReport.html 
Carlson, N. M., Kaplan, B. L., \& Einerson J. J. (March 2000). FY99 professional development fund allocation (INEEL/INT-2000-00319). Idaho Falls, ID: Idaho National Engineering and Environmental Laboratory.

Carlson, N. M. \& McCaslin, M. L. (in press). Meta-Inquiry: An approach to interview success. Qualitative Report, 8(4).

Carlson, N. M., May, W. E., Loertscher, R., \& Cobia, C. (2003). Apprenticeship: Applications in adult education. MPAEA Journal of Education, 17(1), 29-43.

Carnoy, M. (2000). The changing world of work in the information age. In Parkay, F. W. \& Hass, G. (Eds.), Curriculum planning a contemporary approach $\left(7^{\text {th }}\right.$ ed., $p$. 64-68) Needham, MA: Allyn and Bacon.

Carter-Scott, C. (2000). Negaholics ${ }^{\circledR}$ no more! Shawnee Mission, KS: National Press Publications.

Cohen, W. M. \& Levinthal, D. A. (1990). Absorptive capacity: A new perspective on learning and innovation. Administrative Science Quarterly, 35, 128-152.

Collard, B. (1998). Career development inside organizations today. Career Planning and Adult Development Journal, 14(3), 5-11.

Covey, S. R. (1989). The 7 habits of highly effective people. New York: Simon and Schuster.

Creswell, J. W. (1994). Research design: Qualitative \& quantitative approaches. Thousand Oaks, CA: Sage.

Creswell, J. W. (1998). Qualitative inquiry and research design: Choosing among five traditions. Thousand Oaks, CA: Sage.

Cunningham, I. (1994). The wisdom of strategic learning: The self managed learning solution. London: McGraw-Hill. 
Denzin, N. K. (1997). Interpretive ethnography: Ethnographic practices for the $21^{\text {st }}$ century. Thousand Oaks, CA: Sage.

Dirkx, J. M. \& Prenger, S. M. (1997). Planning \& implementing instruction for adults: A theme-based approach. San Francisco: Jossey-Bass.

Dixon, N. M. (1990). Evaluation: A tool for improving HRD quality. San Diego: University Associates.

Dixon, N. M. (1998) Dialogue at work. London: Lemos \& Crane.

Dixon, N. M. (2000). Common Knowledge: How companies thrive by sharing what they know. Boston: Harvard Business Press.

Donoghue, L. P., Harris, J. G., \& Weitzman, B. A. (1999). Knowledge management strategies that create value. Outlook, 11(1), 48-53.

Drucker, P. F. (1999, March-April). Managing oneself. Harvard Business Review. 64-74

Duderstadt, J. J. (1999-2000, Winter). New roles for the 21st-century university. Issues In Science And Technology, 37-44.

Dwyer, D. (1998). How to de-junk your life. Shawnee Mission, KS: National Press Publications.

Eisner, E. W. (2000). Cognition and representation: A way to pursue the American dream? In Parkay, F. W. \& Hass, G. (Eds.), Curriculum Planning A Contemporary Approach ( $7^{\text {th }}$ ed., pp. 248-263). Needham, MA: Allyn and Bacon.

Ford, D. N., Voyer, J. J., \& Wilkinson, J. M. G. (2000). Building learning organizations in engineering cultures: Case study. Journal of Management in Engineering, 16(4), 72-83.

Forrester, J. W. (1965). A new corporate design. Sloan Management Review, 7, 5-17.

Forsyth, D. R. (1999). Group dynamics ( $3^{\text {rd }}$ ed.). Belmont, CA: Wadsworth. 
Fraser, D. (1999). QSR NUD*IST Vivo reference guide (2 ${ }^{\text {nd }}$ ed.) [Reference Guide]. Melbourne, Australia: Qualitative Solutions and Research Pty. Ltd.

Friedland, A. J. \& Folt, C. L. (2000). Writing successful science proposals. New Haven, CT: Yale University Press.

Funderstanding. (2003). Right brain vs. left brain. Retrieved February 8, 2003, from http://www.funderstanding.com/right_left_brain.cfm

Gelatt, H. B. (1998). Self, system, synergy: A career-life development framework for individuals and organizations. Career Planning and Adult Development Journal, 14(3), 13-23.

Glaser, B. G. (1964). Organizational scientists: Their professional careers. Indianapolis, IN: The Bobbs-Merrill.

Glaser, B. G. (1978). Theoretical sensitivity. Mill Valley, CA: Sociology Press.

Glaser, B. G. (1992). Emergence vs. forcing: Basics of grounded theory analysis. Mill Valley, CA: Sociology Press.

Glaser, B. G. (2001). The grounded theory perspective: Conceptualization contrasted with description. Mill Valley, CA: Sociology Press.

Glaser, B. G. \& Strauss, A. (1967). The discovery of grounded theory: Strategies for qualitative research. Chicago: Aldine.

Golden, K. A. (1992). The individual and organizational culture: Strategies for action in highly-ordered contexts. Journal of Management Studies, 29(1), 1-21.

Guba, E. G. \& Lincoln, Y. S. (1981). Effective evaluation. San Francisco: Jossey-Bass.

Halpren, D. F. (1996). Thought and knowledge: An introduction to critical thinking $\left(3^{\text {rd }}\right.$ ed.). Mahwah, NJ: Lawrence Erlbaum Associates. 
Henning, K. (2003, February). Brevity isn't enough you need to write tight. Harvard Management Communication Letter, 4-6.

Henry, J. (1998). Documenting contributory expertise: The value added by technical communicators in collaborative writing situations. Technical Communication, 45(3), 207-220.

Hofstede, G. (1997). Cultures and organizations-software of the mind: Intercultural cooperation and its importance for survival. New York: McGraw-Hill.

Howell, J. M. \& Avolio, B. J. (1993). Transformational leadership, transactional leadership, locus of control, and support for innovation: Key predictors of consolidated-business-unit performance. Journal of Applied Psychology, 78(6), 891-902.

Hughes, M. (2002). Moving from information transfer to knowledge creation: A new value proposition for technical communicators. Technical Communication, 49(3), 275-285.

Johnson, J. \& Pratt, D. D. (1998). The apprenticeship perspective: Modeling ways of being. In D. D. Pratt (Ed.), Five perspectives on teaching in adult and higher education (pp. 83-103). Malabar, FL: Robert E. Krieger.

Johnson-Holloway, P. (2001). Exploring the nature of the phenomenon of stress as it relates to nursing students. Unpublished doctoral dissertation, University of Idaho, Moscow, ID.

Jones, M. B. \& Cooke. P. (1998). Navigating corporate culture change: A career development perspective. Career Planning and Adult Development Journal, 14(3), 43-54. 
Kaplan, B. L. (1999). The implementation of a self-managed work team: An action science investigation (INEEL/EXT-99-01113). Idaho Falls, ID: Idaho National Engineering and Environmental Laboratory.

Kaplan, R. S. \& Norton, D. P. (1993, September-October). Putting the balanced scorecard to work. Harvard Business Review, 134-147.

Keller, R. T. (1992). Transformational leadership and the performance of research and development groups. Journal of Management, 18(3), 489-501.

Kent-Drury, R. (2000, February/March). Bridging boundaries, negotiating differences: The nature of leadership in cross-functional proposal-writing groups. Technical Communication, 90-98.

Krassen-Maxwell, E. (1996). Modeling life: The dynamic relationship between elder modelers and their protégés. In B. G. Glaser (Ed.) assisted by W. D. Kaplan, Gerund grounded theory: The basic social process dissertation (pp. 51-59). Mill Valley, CA: Sociology Press.

Kurstedt, H. A. \& Mallak, L. A. (1996). Understanding and using empowerment to change organizational culture. Industrial Management, 38(6), 159-163.

Kurstedt, H. A., Jr., Mallak, L. A., Howard, E. M., \& Kurstedt, P. S. (1990). Why engineers must know and manage organizational culture. IEEE International Engineering Management Conference: Theme, management through the year 2000-gaining the competitive advantage (pp. 159-163). Santa Clara: CA.

Lave, J. \& Wenger, E. (1991). Situated learning-legitimate peripheral participation. Cambridge, UK: Cambridge University Press.

Lawrence-Lightfoot, S. \& Davis, J. H. (1997). The art and science of portraiture. San Francisco: Jossey-Bass. 
Lencioni, P. (2000). Obsessions of an extraordinary executive: The four disciplines at the heart of making any organization world class. San Francisco: Jossey-Bass.

Liedtka, J. (1999). Linking competitive advantage with communities of practice. Journal of Management Inquiry, 8(1), 5-16.

Margolis, F. H. \& Bell, C. R. (1989) Understanding training perspectives and practices. San Diego: University Associates.

Maxwell, J. C. (2002). The 17 essential qualities of a team player. Nashville, TN: Thomas Nelson, Inc.

McCaslin, M. L. (1995, July). The nature of leadership within rural communities: A grounded theory. In Challenging boundaries in leadership education:

Proceedings of the Association of Leadership Educators. Colorado Springs, CO.

McCaslin, M. L. (1996). Reciprocating transformational leadership. The Journal of Leadership Studies, 3(1), 25-36.

McCaslin, M. L. (2002). Human ecology. Lecture and paper presented to University of Idaho adult education Adult Learner graduate class in February 21, 2002, Idaho Falls, ID.

McCaslin, M. L. \& Scott K. W. (2003). Communities of teaching and learning: The nature of metagogy. Manuscript submitted for publication.

McCaslin, M. L., Scott K. W., \& Carlson, N. M. (2002). Method for studying a human ecology: an adaptation of the grounded theory tradition. Manuscript submitted for publication.

McDaniel, M. A., Schmidt, F. L., \& Hunter, J. E. (1988). Job experience correlates to job performance. Journal of Applied Psychology, 73(2), 327-330. 
McDonough, E. F., III. (2000). An investigation of factors contributing to the success of cross-functional teams. The Journal of Product Innovation Management, 17(3), $221-235$.

McIsaac, G. F. \& Morey, N. C. (1998). Engineers' role in sustainable development: Considering cultural dynamics. Journal of Professional Issues in Engineering Education and Practice, 124(4), 110-119.

McKenna, C. (1997). Powerful communication skills: How to communicate with confidence, clarity and credibility. Shawnee Mission, KS: National Press Publications.

Mead, J. (1998). Measuring the value added by technical documentation: A review of research and practice. Technical Communication, 45(3), 353-379.

Merton, R. K. (1957). Priorities in scientific discovery: A chapter in the sociology of science. American Sociological Review, 22(6), 635-659.

National Press Publications (Ed.). (2001). The manager's role as coach. Shawnee Mission, KS: National Press Publications, Inc.

Odum, E. P. (1959). Fundamentals of ecology. Philadelphia: W. B. Saunders.

Palmer, P. J. (1998). The courage to teach: Exploring the inner landscape of a teacher's life. San Francisco: Jossey-Bass.

Parks Daloz, L. A. (1990). Mentorship. In M. W. Galbraith (Ed.), Adult learning methods (pp. 205-224). Malabar, FL: Robert E. Krieger.

Partridge, R. (1996). Post-licensure baccalaureate education for registered nurses. In B. G. Glaser (Ed.) assisted by W. D. Kaplan, Gerund grounded theory: The basic social process dissertation (pp. 73-84). Mill Valley, CA: Sociology Press. 
Patton, M. Q. (1987). How to use qualitative methods in evaluation. Newbury Park: CA, Sage.

Pedler, M. \& Aspinwall, K. (1998). A concise guide to the learning organization. London: Lemos \& Crane.

Pedler, M., Burgoyne, J., \& Boydell, T. (1997). The learning company. London: The McGraw-Hill.

Penrose, A. M. \& Katz, S. B. (1998). Writing in the sciences: Exploring conventions of scientific discourse. New York: St. Martin's Press.

Polanyi, M. (1983). The tacit dimension. Gloucester, MA: Peter Smith.

Pratt, D. D. \& Associates. (1998). Five perspectives on teaching in adult and higher education. Malabar, FL: Robert E. Krieger.

Premont, S. B. (1990). Internship. In M. W. Galbraith (Ed.), Adult learning methods (pp. 329-344). Malabar, FL: Robert E. Krieger.

Redish, J. (1995). Adding value as a professional technical communicator. Technical Communication, 42(1), 26-39.

Revans, R. (1998). ABCs of action learning. London: Lemos \& Crane.

Robey, D., Khoo, H. M., \& Powers, C. (2000, February/March). Situated learning in cross-functional virtual teams. Technical Communication, 51-66.

Robinson, D. G. \& Robinson, J. C. (1990). Training for impact. San Francisco: JosseyBass.

Rost, J. C. (1991). Leadership for the twenty-first century. New York: Praeger.

Rubin, J. R. \& Rubin, I. S. (1995). Qualitative interviewing: The art of hearing data. Thousand Oaks, CA: Sage. 
Senge, P. M. (1990). The fifth discipline: The art \& practice of the learning organization, New York: Currency Doubleday.

Schein, Edgar H. (1993, Winter). How can organizations learn faster? The challenge of entering the green room. Sloan Management Review, 85-92.

Scott, K. W. (2002). "High self-efficacy and perseverance in adults committed to new challenging life pursuits after age 50: A grounded theory study." Unpublished doctoral dissertation, University of Idaho, Idaho Falls, ID.

Spradley, J. P. (1979). The ethnographic interview. Fort Worth, TX: Harcourt College Publishers.

Spradley, J. P. (1980). Participant observation. Fort Worth, TX: Harcourt College Publishers.

Strauss, A. \& Corbin, J. (1990). Basics of qualitative research: Techniques and procedures for developing grounded theory. London: Sage.

Storck, J. \& Hill P. A. (2000, Winter). Knowledge diffusion through "strategic communities". Sloan Management Review, 63-74.

Tashakkori, A. \& Teddlie, C. (1998). Mixed methodology: Combining qualitative and quantitative approaches. Thousand Oaks: CA, Sage.

Thornton, T. C., Mattocks, C., \& Thornton, L. (2000-2001, Winter). Empowerment: A method of motivating adult learners. Journal of Adult Education, 29(1), 1-10.

T'Kenye, C. (1998). The nurturing perspective: Facilitating self-efficacy. In D. D. Pratt (Ed.), Five perspectives on teaching in adult and higher education (pp. 151-172). Malabar, FL: Robert E. Krieger Publishing Co.

Wenger, E. (1998). Communities of practice: Learning, meaning, and identity. Cambridge, UK: Cambridge University Press. 
Wenger, E. (1999). Communities of practice the key to knowledge strategy. Knowledge Directions, 1(2), 48-63.

Wenger, E. (2000a). Communities of practice: Stewarding knowledge. In C. Despres (Ed.) Knowledge horizons: The present and the promise of knowledge. Woburn, MA: Butterworth-Heinemann Ltd.

Wenger, E. (2000b). Communities of practice and social learning systems. Organization, 7(2), 225-246.

Wenger, E. \& Snyder, W. M. (2002). Communities of practice: The organizational frontier. Boston: Harvard Business School Press.

Wick, C. (2000). Knowledge management and leadership opportunities for technical communicators. Technical Communication, 47(4), 515-529.

Wolcott, H. F. (1998). The elementary school principal. In J. W. Creswell, Qualitative inquiry and research design: Choosing among five traditions (pp. 323-356). Thousand Oaks, CA: Sage.

Wolff, M. F. (1991). Building high-performing R\&D teams collaborate and interactprofitably. Research-Technology Management, 34(5), 11-15.

Woren, N. A. M., Ruddle, K., \& Moore, K. (1999). From organizational development to change management. Journal of Applied Behavioral Science, 35(3), 273-287. 